\title{
Development of turbulence in subsonic submerged jets
}

\author{
Polina S. Landa ${ }^{\mathrm{a}}$ and P.V.E. McClintock ${ }^{\mathrm{b}}$ \\ ${ }^{a}$ Department of Physics, Lomonosov Moscow State University, \\ 119899 Moscow, Russia \\ ${ }^{\mathrm{b}}$ Department of Physics, Lancaster University, Lancaster LA1 4YB, UK
}

\begin{abstract}
The development of turbulence in subsonic submerged jets is reviewed. It is shown that the turbulence results from a strong amplification of the weak input noise that is always present in the jet nozzle exit section. At a certain distance from the nozzle the amplification becomes essentially nonlinear. This amplified noise leads to a transition of the system to a qualitatively new state, which depends only slightly on the characteristics of the input noise, such as its power spectrum. Such a transition has much in common with nonequilibrium noise-induced phase transitions in nonlinear oscillators with multiplicative and additive noise. The Krylov-Bogolyubov method for spatially extended systems is used to trace the evolution of the power spectra, the root-mean-square amplitude of the turbulent pulsations, and the mean velocity, with increasing distance from the nozzle. It is shown that, as turbulence develops, its longitudinal and transverse scales increase. The results coincide qualitatively and also, in specific cases, quantitatively, with known experimental data.
\end{abstract}




\section{Contents}

1 Introduction 3

2 Brief review of the evolution of views of turbulence as an oscillatory process 5

$3 \quad$ Some experimental results concerning turbulence development in jets 10

$\begin{array}{lll}3.1 & \text { The main properties of jet flows } & 10\end{array}$

3.2 Evolution of power spectra of the pulsations of fluid velocity and pressure with the distance from the nozzle exit section 11

3.3 A jet as an amplifier of acoustic disturbances

3.4 Suppression and intensification of turbulence in jets by a weak periodic forcing

4 The analogy between noise-induced oscillations of a pendulum with randomly vibrated suspension axis and turbulent processes in a jet

5 The main equations and dynamics of a plane jet

6 The derivation of truncated equations for the amplitude of stochastic constituents

6.1 Generative solutions

6.2 The first approximation

6.3 The second approximation

$\begin{array}{lll}7 & \text { Conclusions } & 56\end{array}$

$\begin{array}{ll}\text { References } & 57\end{array}$ 


\section{Introduction}

There is an abundance of published works relating to hydrodynamic turbulence problems. It is interesting that the first experimental work where the transition to turbulence was observed as the fluid viscosity decreased, due to heating, was reported in 1839 by Hagen [1]. Over the years the volume of experimental works has increased to such an extent that it cannot even be listed in a review of this kind. As examples, we mention only a fundamental paper by Reynolds [2], where elegant experiments with stained liquid were described and intermittent behavior was first discovered, the Compte-Bellot's book [3], wherein a detailed comparison is made between turbulence power spectra in a plane channel and Kolmogorov's spectra, and the book by Ginevsky et al. [4] in which experiments with jets are reviewed. A wide variety of books is devoted to the problem of hydrodynamic instability playing the major role in the transition to turbulence (see, e.g. [5-10]). Among the many general texts we mention [11-17]. A number of books and a plethora of papers are devoted to numerical calculations of turbulence by both direct and indirect methods (see, e.g. the books [18-21]). An important place in the literature is occupied by studies in which the general properties of so-called fully developed turbulence are derived and investigated. Thus Kolmogorov and Obukhov [22-24], for example, derived the power spectra of developed isotropic turbulence starting from simple dimensional arguments (see also [25]). Different generalizations and refinements of these results were achieved by Novikov [26], Procaccia et al. [27-36], Amati et al. [37] and many other researchers. Recent works, developing an approach to turbulence in the context of contemporary theoretical physics, including field-theoretic and group-theoretic methods, can be also assigned to this class. Among these we mention [38-40].

It is known that, as distinct from flows in channels, jet flows are rarely, if ever, laminar. Over a wide range of Reynolds numbers, so-called hydrodynamic waves are excited and amplified in the body of the jet. The amplitude of these waves decreases exponentially outside the jet shear layer. Undamped hydrodynamic waves can propagate only downstream with a velocity of the order of the flow velocity. The distinctive feature of hydrodynamic waves is their random character. Nevertheless, against the background of this randomness there are comparatively regular large scale patterns known as coherent structures. 
It is very important to note that, when hydrodynamic waves interact with an obstacle or inhomogeneity, they do not undergo simple reflection. Rather, they induce acoustic waves that propagate upstream. The acoustic waves coming up against an obstacle or an inhomogeneity, in their turn, induce hydrodynamic waves propagating downstream, and so on. Owing to these transformations feedback occurs in jet flows, and can excite self-oscillations. Just such a phenomenon arises in jets impinging upon e.g. a flat plate, a wedge, a coaxial ring or a coaxial pipe [41-45]. In free jets inhomogeneities formed by vortices also induce acoustic waves, again resulting in feedback [46]. But this feedback is nonlinear, and it cannot cause the self-excitation of oscillations. Nonetheless, it exerts an influence on the development of turbulence and coherent structures.

As will be shown below, the turbulent character of jet flows is caused by strong amplification of the random disturbances which are always present at the jet nozzle exit section ${ }^{1}$. At a certain distance from the nozzle the amplification becomes inherently nonlinear. The amplification transforms the system to a qualitatively new state which depends only slightly on the power spectrum or other characteristics of the input disturbances. The system behaves much as though it had undergone a phase transition.

The hypothesis that the onset of turbulence can usefully be considered as a noise-induced phase transition was first offered in [47]. It was based on the existence of profound parallels between turbulent processes in nonclosed fluid flows and noise-induced oscillations in a pendulum with a randomly vibrated suspension axis, which undergoes such a phase transition $[44,45,48-51]$. Note that this hypothesis is in contradistinction with the widespread belief that the transition to turbulence arises through the excitation of self-oscillations, first periodic and then chaotic [11,12]; but the latter idea does not explain the origin of the feedback mechanism responsible for exciting the self-oscillations. It is well known that instability in a nonclosed fluid flows is of a convective character, but not absolute. Such an instability cannot excite self-oscillations because all disturbances drift downstream ${ }^{2}$.

$\overline{1}$ It should be noted that random sources are present at all points of a jet, even with no external disturbances - i.e. the so called natural fluctuations [16]. But their influence is significantly less than that of disturbances at the jet nozzle exit section and they can therefore be ignored.

2 It should be noted, however, that the instability of a jet flow in counter-current stream is of an absolute character and can result in self-excited oscillations. 
An extremely interesting manifestation of nonlinear effects in jets lies in the possibility of exploiting them to control turbulence with the aid of acoustic waves applied at some appropriate frequency ([4,52-57]. Similar control of noise-induced oscillations was demonstrated for the harmonically driven pendulum $[58,51]$.

Through an approximate solution of the Navier-Stokes equations based on the KrylovBogolyubov asymptotic method, we will show that explicit consideration of the amplification of the input noise allows us to account for many known experimental results within the initial part of a jet [59]. Moreover, it follows from our theory that the commonly accepted $[60-65,21,4]$ explanation for the well-known shift of velocity pulsation power spectra towards the low-frequency region is in fact erroneous. According to this explanation, the shift of the power spectra occurs because of feedback via an acoustic wave nascent where vortex pairing occurs, as seen in experiments. We will show that the reason for the spectral shift lies in the jet's divergence; and that this shift causes the increase of spatial scale with increasing distance from the nozzle, and results in the observed vortex pairing.

It should be noted that interesting phenomena similar to those for ordinary hydrodynamic turbulence are also observed in flows of superfluid helium [66].

\section{Brief review of the evolution of views of turbulence as an oscillatory process}

It is well known that fluid flow in channels is laminar for small flow velocities and turbulent for large flow velocities $[11,12,9]$. The problem of how turbulence originates has long attracted the considerable attention of researchers. As is known from the Rytov memoirs [67], the Russian physicist Gorelik believed that

“...turbulence with its threshold of 'self-excitation', with typical hysteresis in its appearance or disappearance as the flow velocity increases or decreases, with paramount importance of nonlinearity for its developed (stationary) state — is self-oscillations. Their specific character lies in that they are self-oscillations in a continuous medium, i.e. in a system with very large number of degrees of freedom." 
Landau held implicitly the same viewpoint. According to Landau turbulence appears in the following manner: first the equilibrium state corresponding to laminar flow becomes unstable and self-oscillations with a single frequency are excited. To describe the amplitude of these self-oscillations, based on physical considerations, Landau wrote a phenomenological equation similar to the truncated van der Pol equation for the amplitude of self-oscillations in a vacuum tube generator, commenting [68]: "With further increase of the Reynolds number new periods appear sequentially. As for the newly appeared motions, they have increasingly small scales". As a result, multi-frequency self-oscillations with incommensurate frequencies, i.e. quasi-periodic motion, must set in. An attractor in the form of a multi-dimensional torus in the system phase space has to be associated with these self-oscillations. For a large number of frequencies such quasi-periodic self-oscillations differ little in appearance from chaotic ones, which is why developed turbulence is perceived as a random process. In spite of the fact that Landau's theory was phenomenological, and did not follow from hydrodynamic equations, it was accepted without question for a long time by almost all turbulence researchers. Moreover, this theory was further developed by Stuart [69-72] who proposed a technique for calculating the coefficients involved in the Landau equations, based on an approximate solution of the Navier-Stokes equations. However, the approximate solution sought by Stuart in the form of $A(\epsilon t) e^{i(\omega t-k x)}$ is, from a physical standpoint, incorrect. It describes a wave that is periodic in space, with a given wave number $k$ and with a slowly time varying amplitude $A(\epsilon t)$. Strictly speaking such a solution is true only for a ring flow of length $L=2 \pi n / k$, where $n$ is an integer, i.e. for a flow with feedback. We note that a similar approach to hydrodynamic instability was used by many scientists, beginning from Heisenberg [73].

In the 1970s, after the discovery of the phenomenon of deterministic chaos and the realization that a multi-dimensional torus is unstable [74], the Landau theory became open to question, but the conception of self-oscillations was retained. The difference lay only in that, instead of quasi-periodic self-oscillations, they became spoken of as chaotic ones. Thus, according to these new ideas, the onset of turbulence is the sudden birth of a strange attractor in the phase space of certain dynamical variables [74,75]. We note that similar ideas were repeatedly expressed by Neimark (see [76]). Using the concept of turbulence as self-oscillations, Gaponov-Grekhov and with co-workers published several articles on the 
simulation of turbulence, modelled in a chain of coupled oscillators $[77,78]$.

However, we believe that turbulence arising in nonclosed fluid flows is not a self-oscillatory process. As already mentioned above, the instability of nonclosed laminar flows is of a convective character but not absolute. This means that a disturbance arising at some point of the flow will not increase indefinitely with time, but will drift downstream. It follows from this property of convectively unstable systems that they are not self-oscillatory, but are amplifiers of disturbances ${ }^{3}$. For such a system to become self-oscillatory, global feedback must be introduced, e.g. by closing the system in a ring ${ }^{4}$. Disturbances are necessarily present in all real systems, both from external sources (technical fluctuations) and as a result of the molecular structure of a substance (natural fluctuations). The disturbances can be included as external forces in equations describing the system behavior. The calculation of the forces caused by the natural fluctuations in hydrodynamic flows, based on the fluctuation-dissipation theorem, was performed by Klimontovich [16]. In hydrodynamic flows the presence of fluctuations, especially at the input, is crucial because they are precisely what lead eventually to the turbulent disturbances observed. It follows from this that an approach to turbulence within the framework of (deterministic) dynamical systems theory is not always appropriate.

Naturally, the question arises as to how to treat the features of turbulence which, as pointed out by Gorelik, are seemingly precisely those that are inherent in self-oscillatory systems. First, the term "self-excitation" should be replaced by "loss of stability". Furthermore, the hysteresis of turbulence, its "appearance or disappearance as the flow velocity increases or decreases" can be explained in terms of the specific character of the nonlinearity of the gain factor. Finally, the "paramount importance of nonlinearity for its fully developed (stationary) turbulent state" is quite possible in amplifiers too, because nonlinearity of the amplifier can have considerable influence on its output power spectrum.

One piece of evidence suggesting that turbulence is not a self-oscillatory process comes from the numerical experiments of Nikitin $[80,81]$. He simulated fluid flow in a circular

\footnotetext{
3 This fact was first mentioned by Artamonov [79].

${ }^{4}$ In essence, this is exactly what occurs in the process of numerical simulation with periodic boundary conditions.
} 
pipe of a finite length and radius $R$ with a given velocity at the input cross-section, and with so-called 'soft' boundary conditions at the output cross-section; these latter are

$$
\frac{\partial^{2} u}{\partial x^{2}}=\frac{\partial^{2} \xi}{\partial x^{2}}=\frac{\partial^{2} \eta}{\partial x^{2}}=0
$$

where $u$ is the longitudinal velocity component, $\xi$ and $\eta$ are the radial and angular components of vorticity $\boldsymbol{\Omega}=\operatorname{rot} \mathbf{u}, \mathbf{u}=\{u, v, w\}$ is the flow velocity vector in cylindrical coordinates $x, r$ and $\theta$. Under these conditions a reflected wave apparently does not appear, or is very weak.

At the input cross-section of the pipe the longitudinal velocity component was taken to be in the form of the Poiseuille profile $u_{0}\left(1-r^{2} / R^{2}\right)$, weakly disturbed by a harmonic force at the frequency $\omega=0.36 u_{0} / R$, i.e.,

$$
\begin{aligned}
& u=u_{0}\left(1-\frac{r^{2}}{R^{2}}\right)+A \operatorname{Re}\left(u^{\prime}(r) e^{-i \omega t}\right) \cos \theta \\
& v=A \operatorname{Re}\left(v^{\prime}(r) e^{-i \omega t}\right) \cos \theta, \quad w=A \operatorname{Re}\left(w^{\prime}(r) e^{-i \omega t}\right) \sin \theta,
\end{aligned}
$$

where $u^{\prime}(r), v^{\prime}(r)$ and $w^{\prime}(r)$ are the components of the Orr-Sommerfeld vector-eigenfunction at frequency $\omega, R$ is the pipe radius, and $A$ is the disturbance amplitude. The velocity $u_{0}$ and the pipe radius $R$ were set such that the Reynolds number Re was equal to 4000 . As the amplitude $A$ exceeded a certain critical value $\left(A \geq A_{\mathrm{cr}}\right)$, random high-frequency pulsations appeared in the flow after a short time interval. They occupied all the lower part of the pipe from $x=x_{0}$, where $x_{0}$ depended only weakly on the distance $r$ from the pipe axis. It turned out that the value of $x_{0}$ decreased as $A$ became larger. The appearance of turbulent pulsations was accompanied by corresponding deformation of the profile of the longitudinal constituent of the mean velocity: at the pipe axis the mean velocity decreased, whereas near the pipe wall it increased. We note that a similar deformation of the mean velocity profile with increasing turbulent pulsations occurs in jet flows as well. The instantaneous distributions of the longitudinal velocity component in a steady regime for $A / u_{0}=0.04$ are shown in Fig. 1 [81]. 
As the amplitude $A$ gradually decreased, the turbulent region drifted progressively downstream and disappeared at a certain value of $A$. It is known $[82,8]$ that Poiseuille flow in a circular pipe, in contrast to that in a plane channel, possesses the property that laminar flow is stable with respect to small perturbations for any Reynolds number. However, in the case of sufficiently large Reynolds numbers, such a flow is unstable with respect to finite perturbations. If an attractor existed corresponding to the turbulent mode, and if the role of the harmonic disturbance was to lead phase trajectories into the attractor basin, then turbulence should not disappear following cessation of the harmonic disturbance.

It may be inferred from Fig. 1 that the development of turbulence for $A \geq A_{\text {cr }}$ is associated with a peculiar phase transition at the point $x=x_{0}$ induced by an amplification of the noise that is always present in any numerical experiment owing to rounding errors. The harmonic disturbance plays a dual role. First, it causes the appearance of instability and, secondly, it initiates the phase transition, much as occurs in a pendulum with a randomly vibrated suspension axis [58], or in jets under low-frequency acoustic forcing [4]. It is no accident that the transition to turbulence was observed by Nikitin only for low-frequency disturbances (for Strouhal numbers of order 0.1).

Possible counter-arguments against the above ideas lie in the fact that numerical simulation results obtained with periodic boundary conditions are very close to those observed experimentally. But the data obtained by Nikitin in the numerical experiment described above are also close to numerical data for periodic boundary conditions [80]. The visual similarity of turbulent pulsations calculated for periodic conditions, and for the boundary conditions (2.1), (2.2), is illustrated in Fig. 2 [45]. This similarity may be explained by the fact that many nonlinear oscillatory systems possess such pronounced intrinsic properties that they exhibit these properties independently of the means of excitation. Some examples of such (non-hydrodynamic) systems are described in [83].

Note that our discussion is not related to so called closed flows, e.g. to the Couette flow between two rotating cylinders or spheres (see [44]). In closed flows there is always feedback linking the output of the amplifier to its input, so that they consequently become self-oscillatory. 


\section{Some experimental results concerning turbulence development in jets}

\subsection{The main properties of jet flows}

Issuing from a nozzle, a fluid jet always noticeably diverges. This is associated with the fact that, owing to viscosity, neighboring fluid layers are increasingly drawn into the motion. This phenomenon has come to be known as entrainment. The profile of the flow velocity changes essentially in the process. At the nozzle exit, it is nearly rectangular, whereas away from the nozzle it becomes bell-shaped: see Fig. 3 a. The fluid layer within which the mean velocity changes significantly is called the shear layer or the mixing layer (see, for example, $[84,86,87])$. It can be seen from Fig. $3 a$ that, within the initial part of the jet $\left(x \leq x_{\text {in }}\right)$, the thickness of the mixing layer increases with increasing distance from the nozzle. At $x=x_{\text {in }}$ the thickness of the internal part of the mixing layer $\delta_{1}$ becomes equal to the half-width of the nozzle outlet for a plane jet, or the nozzle radius for a circular jet, whereupon a continuous boundary layer is formed. In the vicinity of the jet axis, the mean velocity first decreases very slowly with increasing distance $x$ from the nozzle. This part of the jet is called the initial part: see I in Fig. $3 b$. Further on, the decrease of the mean velocity becomes significant. This part of the jet is called the main part: see III in Fig. 3 b. Parts I and III are separated by the so-called transient part II. The length of the initial part decreases with increasing intensity of disturbances at the nozzle exit section. This can be seen in Fig. 4, where experimental dependences of the relative mean velocity $U / U_{0}$ on the relative distance $x / D$ from nozzle are plotted ( $D$ is the nozzle diameter). Results are shown for three values of the intensity of the disturbances at the nozzle exit section $\epsilon_{u}(0)=\sqrt{\overline{u(0)^{2}}-U(0)^{2}} / U_{0}$, where $U_{0}$ and $u(0)$ are the mean velocity and longitudinal component of total flow velocity at the center of nozzle exit section, respectively $[4,21]$.

The main parts of plane and axially symmetric jets possess approximately the property of self-similarity, i.e. at all jet cross-sections the velocity profiles are affine-similar [14]. For a plane jet the property of self-similarity means that the jet velocity can be presented in the form $u(x, y)=x^{-\alpha} F\left(y / x^{\beta}\right)$, where $x$ and $y$ are longitudinal and transverse coordinate respectively, $\alpha$ and $\beta$ are certain numbers and $F$ is a function of $y / x^{\beta}$. The processes in the jet main part are studied in considerable detail (see, e.g. [85-93]). We will consider 
only the processes in the initial part of a jet.

It is interesting that coherent structures are formed just in the mixing layer of initial part of the jet . They are vortex formations (bunches of vorticity). Their sizes are of the order of the thickness of the shear layer, and they are moderately long-lived. The presence of coherent structures in a jet shear layer results in the intermittent behavior of a jet flow, especially in the neighborhood of the external boundary of a jet, where turbulent and laminar phases alternate [94].

\subsection{Evolution of power spectra of the pulsations of fluid velocity and pressure with the distance from the nozzle exit section}

The randomness of the hydrodynamic waves excited in a jet manifests itself, in particular, as continuous power spectra of the pulsations of fluid velocity and pressure. Within the initial part of the jet, these spectra are of a resonant character. Experiments show that the frequency $f_{\mathrm{m}}$ corresponding to the maximum of the power spectrum within the initial part of the jet decreases as the distance from the nozzle exit increases [95-97,50]. Within the main part of the jet, the power spectra decrease monotonically with frequency. Fig. 5 shows examples of how the power spectra of the velocity pulsations evolve with distance from the nozzle exit, along the jet axis, and along a line offset by $R$ from the axis [50]. The abscissa in each case plots the frequency expressed in terms of the Strouhal numbers $\mathrm{St}=f D / U_{0}$.

As mentioned above, most studies of the different processes in jets attribute such behavior of the pulsation power spectrum within the mixing layer to a pairing of vortices. When pairing takes place, the vortex repetition rate must be halved. Within the initial part of the jet, depending on the conditions of outflow, from 3 to 4 pairings of vortices are usually observed [61]. The frequency $f_{\mathrm{m}}$ at the end of the jet's initial part should therefore decrease by factor of between 8 and 16, a conclusion that conflicts with experimental data. Experiments show that the frequency $f_{\mathrm{m}}$ is not a step, but a smooth function of distance from the nozzle exit (see Fig. 6, where the experimental dependences of the Strouhal number $\mathrm{St}_{\mathrm{m}}$ on the relative distance from the jet nozzle exit $x / D$ are plotted [45]). In 
an attempt to resolve this conflict, the researchers holding this viewpoint speculate that there is a statistical spread in the sites of pairing, but without explaining why there should be such a spread.

The faster decrease of $\mathrm{St}_{\mathrm{m}}$ within the mixing layer, compared to what happens on the jet axis, may result from the influence of nonlinear feedback caused by acoustic waves induced by vortices within the jet mixing layer. The presence of such waves is indirectly supported by the experimental data of Laufer [61]. According to these data high-frequency pulsations of fluid velocity within a mixing layer near the nozzle exit are modulated by low-frequency pulsations with frequencies corresponding to Strouhal numbers St from 0.3 to 0.5 . This fact can be also illustrated by the power spectrum of velocity pulsations on a line offset by $R$ from the jet axis for $x / D=0.5$ (see Fig. 5 , at the bottom). We see that the spectrum peaks at the main frequency corresponding to the Strouhal number $\mathrm{St}=3.2$ and the two side frequencies corresponding to $\mathrm{St}_{1}=2.7$ and $\mathrm{St}_{2}=3.7$. This means that the modulation frequency corresponds to the Strouhal number 0.5. Owing to the nonlinear feedback, each jet cross-section can be considered as an oscillator with a natural frequency depending on the distance from that cross-section to the nozzle exit. It is evident that the strongest pulsations at the cross-section at coordinate $x$ have to occur at a frequency $f_{m}$ that is related to $x$ by the resonant relation

$$
\frac{x f_{\mathrm{m}}}{U_{v}}+\frac{x f_{\mathrm{m}}}{a}=N
$$

where $U_{v}$ is the velocity of the vortex motion ${ }^{5}, a$ is the sound velocity, and $N$ is an integer. From this it follows at once that $f_{\mathrm{m}} \sim x^{-1}$. This is precisely the dependence which was found experimentally by Petersen [96] (Fig. 6 a). Outside the boundary layer where, within the initial part, inhomogeneities are very weak and nonlinear feedback is nearly absent, the decrease of $f_{\mathrm{m}}$ with increasing $x$ follows from the linear theory and is explained by the jet's divergence as is shown below.

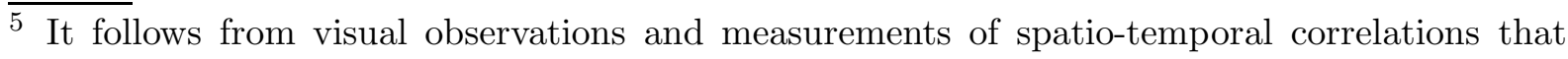
$U_{v} \approx 0.5-0.7 U_{0}$.
} 


\subsection{A jet as an amplifier of acoustic disturbances}

Owing to its strong instability, a fluid jet acts as an amplifier of disturbances whose frequencies lie within a certain range. It is an amplifier with a high spatial gain factor. A small acoustic disturbance at some frequency $f_{\mathrm{a}}$ within this range near the nozzle transforms into a growing hydrodynamic wave. There is evidence for this in the experimental results of Crow and Champagne [98] and Chan [99]. It follows from the experimental data in Fig. 7 [98] that, above a certain value of the acoustic wave amplitude, the dependence of the relative root-mean-square pulsation of the longitudinal component of hydrodynamic velocity $\epsilon_{u}=\sqrt{\overline{u^{2}}} / U_{0}$ acquires a resonant character. Here $u$ is the deviation of the longitudinal component of hydrodynamic velocity from its mean value at the acoustic wave frequency $f_{\mathrm{a}}$, measured in terms of Strouhal numbers. The latter authors consider that the resonance is caused by a combination of linear amplification and nonlinear saturation. The latter increases as the frequency of the disturbance rises. For $\epsilon_{u_{\mathrm{a}}}=\sqrt{\overline{u_{\mathrm{a}}^{2}}} / U_{0}=0.02$, where $u_{\mathrm{a}}$ is the oscillatory velocity in the acoustic wave, the dependence of $\epsilon_{u}$ on $\mathbf{S t}_{\mathrm{a}}=f_{\mathrm{a}} D / U_{0}$ is shown in Fig. 8. We see that $\epsilon_{u}$ is maximal for $\mathbf{S t}_{a} \approx 0.3$.

Figure 9 a taken from [99] shows that the gain factor depends nonmonotonically on distance from the nozzle exit: it has a maximum at $x / D=(0.75 \div 1.25) / \mathbf{S t}_{\mathrm{a}}$. A theoretical dependence similar to that shown in Fig. $9 a$ was found by Plaschko [100] by approximate solution of the linearized Euler equations for a slowly diverging jet. It is depicted in Fig. 9 b. By doing so, Plaschko showed that the decrease of the gain factor away from the nozzle exit is caused by jet divergence, and not by nonlinear effects as was claimed by a number of researchers.

\subsection{Suppression and intensification of turbulence in jets by a weak periodic forcing}

An interesting consequence of the nonlinear effects in a jet is the possibility they provide for controlling the turbulence level and the length of the jet's initial part by application of a weak acoustic wave, or by vibration of the nozzle, at an appropriate frequency [101,4]. In

the case of high-frequency forcing, the hydrodynamic pulsations are suppressed, whereas 
at low frequencies, vice versa, there is intensification of pulsations and turbulence. The experiments show that marked intensification or suppression of turbulence within the initial part of a jet, induced by a periodic forcing, is accompanied by changes in the aerodynamic, thermal, diffusive and acoustic properties of the jet. All of these phenomena have been observed by different researchers.

It should be noted that the influence of acoustic forcing was first studied by Ginevsky and Vlasov [102-106]. Let us consider their main results.

In the case of a low-frequency harmonic acoustic forcing at a frequency $f$ corresponding to a Strouhal number in the range 0.2 -to 0.6 , the vortices in the jet's mixing layer are enlarged within the initial part. In turn, this results in an intensification of the turbulent intermixing, thickening the mixing layer, shortening of the initial part and an increase in entrainment; at the same time, the longitudinal and radial velocity pulsations at the jet axis rise steeply. These effects are observed independently of the direction of the jet irradiation, provided that the amplitudes of the longitudinal and radial components of oscillatory velocity in the sound wave at the jet axis near the nozzle lie in the range 0.05-2\% of $U_{0}$. For the effects to occur, the amplitude of the acoustic wave must exceed a certain threshold value. As the wave amplitude rises above this threshold, turbulent intermixing at first intensifies and the saturates. A further increase of the wave amplitude has little or no effect on the jet.

For high-frequency acoustic forcing of the jet at a frequency corresponding to a Strouhal numbers in the range 1.5-5.0, the vortices in the jet mixing layer become smaller. This results in an attenuation of the turbulent intermixing, a reduction in the thickness of the mixing layer, a lengthening of the initial part, and a decrease of entrainment. Correspondingly, the longitudinal and radial velocity pulsations on the jet axis decrease. In contrast to the effect of low-frequency forcing, high-frequency forcing does not lead to saturation with increasing amplitude; moreover, an increase in the amplitude beyond a certain value causes, not suppression of the turbulence, but its intensification (see Fig. 10 [65,107]). These effects are observed universally for jets over a wide range of Reynolds numbers $\left(\operatorname{Re}=10^{2}-10^{6}\right)$, both for initially laminar and for turbulent boundary layers with a level of initial turbulence less than $10 \%$. 
The foregoing can be illustrated by the experimental dependences of the flow relative mean velocity, and the relative root-mean-square pulsation of the longitudinal $\left(\epsilon_{u}\right)$ and radial $\epsilon_{v}$ components of hydrodynamic velocity on the distance from the nozzle exit along the jet axis for fixed values of the Strouhal number (Fig. 11). All the dependences shown correspond to a fixed value of the acoustic forcing intensity. We see that the mean velocity decreases essentially in the case of low-frequency forcing $\left(0.2<\mathrm{St}_{\mathrm{a}}<1.5\right)$ and increases in the case of high-frequency forcing $\left(S t_{\mathrm{a}}>1.5\right)$. It should be noted that, as the acoustic forcing intensity at low-frequency increases, the initial part of the jet decreases in length right down to the point where it disappears [108].

Effects similar to those described above are also observed for other means of periodic forcing of the jet: e.g. longitudinal or radial vibration of the nozzle, or a pulsating rate of fluid outflow from the nozzle $[65,4]$.

Detailed experimental and numerical studies of turbulence suppression in jet flows were also performed by Hussain and collaborators [109-112]. We concentrate in particular on a single result of these works: that the suppression of turbulence by acoustic forcing of constant amplitude depends on its frequency nonmonotonically: it is maximal at a value of the forcing frequency that depends on the amplitude (see Fig. 12 taken from [112]) ${ }^{6}$.

Periodic forcing of a jet changes markedly the form of its power spectra. For low-frequency forcing, the power spectra of the velocity pulsations near the nozzle contain discrete constituents at the forcing frequency and its higher harmonics. An example of such a spectrum is given in Fig. 13. In the case of high-frequency forcing (see Fig. 14), the power spectra of the velocity pulsations within the jet mixing layer in the immediate vicinity of the nozzle exit also contain discrete components at the forcing frequency and its higher harmonics. At a short distance from the nozzle the second subharmonic appears in the spectrum. Next the fourth, eighth and successively higher subharmonics appear in the spectra. At sufficiently large distances from the nozzle exit the spectra are decreasing almost monotonically.

\footnotetext{
$\overline{6}$ We note that authors of [112] used, not the conventional Strouhal number St, but $\mathrm{St}_{\theta}=\mathrm{St} \theta / D$, where $\theta$ is the so-called boundary layer momentum thickness at the nozzle exit (see [14]).
} 
Kibens [113] obtained the dependences on distance from the nozzle of the Strouhal number corresponding to the spectral line of highest intensity, both along the jet axis and along a line offset from the axis by $R$, for high-frequency acoustic forcing with a Strouhal number of 3.54 (Fig. $15[113,45]$ ). We see that these dependences are step-like, with distinct hysteresis phenomena. Adherents to the viewpoint that the decrease of $\mathbf{S t}_{m}$ with distance from the nozzle for a free jet is caused by vortex pairing attribute the step-like character of the dependences to localization of the sites of pairing caused by the acoustic forcing $[65,4]$. In this explanation, the causes of the localization are ignored and the hysteresis phenomena are not discussed. The picture presented in Fig. 15 can also be interpreted as the successive occurrence of subharmonic resonances of higher and higher order as $x$ increases. The transition from subharmonic resonance of one order to the next can clearly be accompanied by hysteresis, if within a certain range of $x$ both of the resonances are stable. In the transition to a subharmonic resonance of higher order, the frequency has to be halved. This can manifest itself as vortex pairing. We can thus infer that the experimentally observed localization of the sites of vortex pairing, when an acoustic wave acts upon a jet, is a consequence, but not a cause, of the indicated behavior of the power spectra.

\section{The analogy between noise-induced oscillations of a pendulum with ran- domly vibrated suspension axis and turbulent processes in a jet}

It seems at first sight very surprising that there should exist any analogy between the development and control of turbulence in a jet, and the noise-induced oscillations of a pendulum with a randomly vibrated suspension axis. These latter oscillations and their control were first studied in $[114,115,58,116,117,51]$. We believe that the analogy arises because the onset of turbulence in jets is a noise-induced phase transition, and the pendulum with a randomly vibrated suspension axis is an appropriate model for illustrating just such a transition [48].

In the simplest case, when additive noise is neglected, the equation describing the oscil- 
lations of a pendulum with a randomly vibrated suspension axis is:

$$
\ddot{\varphi}+2 \beta\left(1+\alpha \dot{\varphi}^{2}\right) \dot{\varphi}+\omega_{0}^{2}(1+\xi(t)) \sin \varphi=0,
$$

where $\varphi$ is the pendulum's angular deviation from the equilibrium position, $2 \beta\left(1+\alpha \dot{\varphi}^{2}\right) \dot{\varphi}$ is proportional to the moment of the friction force which is assumed to be nonlinear, $\omega_{0}$ is the natural frequency of small oscillations, and $\xi(t)$ is a comparatively wide-band random process with nonzero power spectral density at the frequency $2 \omega_{0}$.

When the intensity of the suspension axis vibration ${ }^{7}$ exceeds a certain critical value proportional to the friction factor $\beta$, excitation of pendulum oscillations occurs, and the variance of the pendulum's angular deviation becomes nonzero. The evolution of such oscillations, and their power spectra with increasing noise intensity, found by the numerical simulation of Eq. (4.1), are shown in Fig. 16. It can be seen from the figure that, close to the excitation threshold, the pendulum oscillations possess the property of so called onoff-intermittency. This notion was first introduced by Platt et al. [118], although a similar phenomenon was considered earlier in [119]. It was noted in [118] that intermittency of this kind is similar to the intermittency in turbulent flows. It is of importance that onoff intermittency is possible, not only in dynamical systems, but in stochastic ones as well [120]. It results from fluctuational transitions through the boundary of excitation $[121,123]$. External manifestations of on-off intermittency are similar to those of ordinary intermittency (see e.g. [124]), i.e. over prolonged periods the pendulum oscillates in the immediate vicinity of its equilibrium position ('laminar phases'); these slight oscillations alternate with short random bursts of larger amplitude ('turbulent phases'). Away from the excitation threshold the duration of the laminar phases decreases and that of the turbulent ones increases, with the laminar phases ultimately disappearing altogether [121]. The variance of the pendulum's angular deviation increases in the process. Comparing the evolution of the power spectra shown in Figs. 5 and 16, we can see that they have much in common.

As described in [122], high-pass filtering of turbulent velocity pulsations reveals their $\overline{7}$ By intensity of the suspension axis vibration is meant the spectral density of $\xi(t)$ at frequency $2 \omega_{0}\left(\kappa\left(2 \omega_{0}\right)\right)$. 
intermittent behavior. We have studied this phenomenon both for experimental velocity pulsations in a jet measured by one of us [50] and also for the pendulum oscillations considered above. In each case we have observed on-off intermittency after high-pass filtering. This fact can be considered as an additional argument in support of the parallels between noise-induced pendulum oscillations and turbulent processes in jets.

It is important to note that the response of the pendulum to a small additional harmonic force (additional vibration of the suspension axis) is similar to the response of a jet to an acoustic force. For example, in the case when the intensity of the random suspension axis vibration is close to its threshold value, the dependence of the intensity of pendulum oscillations on the frequency of the additional harmonic forcing is of a resonant character, very much like a jet subject to an acoustic force (compare Figs. 17 and 8).

Just as in the case of turbulent jets, the noise-induced pendulum oscillations under consideration can be controlled by a small additional harmonic force. The inclusion of the additional force can be effected by substitution into Eq. (4.1) of $\xi+a \cos \omega_{a} t$ in place of $\xi$, where $a$ and $\omega_{a}$ are respectively the amplitude and frequency of the additional vibration of the suspension axis. If the frequency of the additional forcing is relatively low, then this forcing intensifies the pendulum oscillations and lowers the excitation threshold; vice versa, a relatively high-frequency forcing suppresses the pendulum oscillations and increases the excitation threshold. The intensification of the pendulum oscillations by a low-frequency additional vibration is illustrated in Fig. 18 for two values of the vibration frequency. We see that the lower the forcing frequency is, the larger the variance of the oscillation becomes. Just as for jets [125], when the forcing amplitude becomes relatively large, the pendulum's oscillation amplitude saturates.

We now consider in detail the possibility of suppressing noise-induced pendulum oscillations by the addition of a high-frequency vibration. Numerical simulation of Eq. (4.1) with $\xi+a \cos \omega_{a} t$ in place of $\xi$, where $\omega_{a}>2$, shows that such suppression can occur. The results of the simulation are presented in Figs. 19 and 20. It is evident from Fig. 20 that, for small amplitudes of the high-frequency vibration, this vibration has little or no effect on the noise-induced oscillations (see Fig. 19 a). As the amplitude increases, however, the intensity of the noise-induced oscillations decreases rapidly and the duration of the 
'laminar' phases correspondingly increases (see Figs. $19 b, c, d$, and $e$ ). When the amplitude exceeds a certain critical value (for $\omega_{a}=19.757$ it is equal to 42 ) the oscillations are suppressed entirely. As the amplitude increases further the oscillations reappear, but now because the conditions required for parametric resonance come into play. For smaller frequencies $\omega_{a}$, the behavior of the pendulum oscillations is different. The dependences of the variance of the angle $\varphi$ on $a$ for a number of values of the vibration frequency are shown in Fig. 20. It is evident that the variance of $\varphi$ at first decreases, passes through a certain minimum value, and then increases again. It is important to note that this minimum value becomes smaller with increasing forcing frequency, but that it is attained for larger forcing amplitudes at higher frequencies. For sufficiently high frequencies the oscillations can be suppressed entirely (the case illustrated in Fig. 19). The dependence shown in Fig. 20 a closely resembles the corresponding dependence for a jet presented in Fig. 10 .

The dependences of $\sigma$ on $\omega_{a}$ for a number of fixed amplitudes of the additional vibration are illustrated in Fig. 21. Again, these dependences closely resemble the corresponding ones for a jet shown in Fig. 12.

The presence of a small additive noise, in addition to the multiplicative one in Eq. (4.1), does not change the behavior of the pendulum qualitatively, but there are large quantitative differences. The principal one is the impossibility of achieving full suppression of the pendulum oscillations. Nevertheless, a very marked attenuation of the oscillation intensity occurs. This is illustrated in Fig. 22. It should be emphasized that, in the case of turbulence, full suppression is of course also impossible.

\section{The main equations and dynamics of a plane jet}

Let us consider a plane jet issuing from a nozzle of width $2 d$. Neglecting compressibility, we may describe the processes in such a jet by the two-dimensional Navier-Stokes equation for the stream function $\Psi(t, x, y)[11]$ :

$$
\frac{\partial \Delta \Psi}{\partial t}-\frac{\partial \Psi}{\partial x} \frac{\partial \Delta \Psi}{\partial y}+\frac{\partial \Psi}{\partial y} \frac{\partial \Delta \Psi}{\partial x}-\nu \Delta \Delta \Psi=0,
$$


where $\Delta=\frac{\partial^{2}}{\partial x^{2}}+\frac{\partial^{2}}{\partial y^{2}}$ is the Laplacian, $\nu$ is the kinematic viscosity, $x$ is the coordinate along the jet axis, and $y$ is the transverse coordinate. The stream function $\Psi(t, x, y)$ is related to the longitudinal $(U)$ and transverse $(V)$ components of the flow velocity by

$$
U(t, x, y)=\frac{\partial \Psi}{\partial y}, \quad V(t, x, y)=-\frac{\partial \Psi}{\partial x}
$$

We can conveniently rewrite Eq. (5.1) in terms of the stream function $\Psi(t, x, y)$ and the vorticity $\tilde{\Omega}(t, x, y)$ which is defined by

$$
\tilde{\Omega}(t, x, y)=\Delta \Psi(t, x, y)
$$

In dimensionless coordinates $x^{\prime}=x / d, y^{\prime}=y / d$ and time $t^{\prime}=U_{0} t / d$, the equations for the stream function and vorticity become:

$$
\begin{aligned}
& \tilde{\Omega}^{\prime}\left(t^{\prime}, x^{\prime}, y^{\prime}\right)=\Delta^{\prime} \Psi\left(t^{\prime}, x^{\prime}, y^{\prime}\right), \\
& \frac{\partial \tilde{\Omega}^{\prime}\left(t^{\prime}, x^{\prime}, y^{\prime}\right)}{\partial t^{\prime}}-\frac{\partial \Psi^{\prime}\left(t^{\prime}, x^{\prime}, y^{\prime}\right)}{\partial x^{\prime}} \frac{\partial \tilde{\Omega}^{\prime}\left(t^{\prime}, x^{\prime}, y^{\prime}\right)}{\partial y^{\prime}} \\
& +\frac{\partial \Psi^{\prime}\left(t^{\prime}, x^{\prime}, y^{\prime}\right)}{\partial y^{\prime}} \frac{\partial \tilde{\Omega}^{\prime}\left(t^{\prime}, x^{\prime}, y^{\prime}\right)}{\partial x^{\prime}}-\frac{2}{\operatorname{Re}} \Delta^{\prime} \tilde{\Omega}^{\prime}\left(t^{\prime}, x^{\prime}, y^{\prime}\right)=0,
\end{aligned}
$$

where $\Delta^{\prime}$ is the Laplacian in terms of $x^{\prime}$ and $y^{\prime}, \operatorname{Re}=2 U_{0} d / \nu$ is the Reynolds number, and $U_{0}$ is the mean flow velocity in the nozzle center.

From this point onwards the primes will be dropped. It should be noted that in so deciding on a dimensionless time, the circular frequencies $\omega=2 \pi f$ are measured in units of $\mathbf{S}=$ $\omega d / U_{0} \equiv \pi \mathrm{St}$, where $\mathrm{St}=2 f d / U_{0}$ is the Strouhal number.

In accordance with the ideas presented above, the onset of turbulence is caused by random disturbances (noise) in the nozzle exit section. The authors of most of the works devoted to the stability of these small disturbances [126-128,132] split the solution into mean values and small random disturbances. In our opinion this procedure is inappropriate for two reasons: first, exact equations for the mean values are unknown; and, secondly, the random disturbances make a significant contribution to the mean values. Therefore we split the solution of Eqs. (5.4), (5.5) into dynamical and stochastic constituents. The dynamical constituents are described by stationary Navier-Stokes equations and differ 
from the mean values of the corresponding variables because, owing to the quadratic nonlinearity, the stochastic constituents also contribute to the mean values. Ignoring noise sources everywhere except in the nozzle exit (for $x=0$ ) we set

$$
U(t, 0, y)=u_{\mathrm{d}}(0, y)+\xi_{1}(t, y), \quad V(t, 0, y)=v_{\mathrm{d}}(0, y)+\xi_{2}(t, y)
$$

where $u_{\mathrm{d}}(0, y)$ and $v_{\mathrm{d}}(0, y)$ are the dynamical constituents of the longitudinal and transverse velocity components, respectively, and $\xi_{1}(t, y)$ and $\xi_{2}(t, y)$ are random processes. It should be noted that $u_{\mathrm{d}}(0, y)$ and $v_{\mathrm{d}}(0, y)$, as well as $\xi_{1}(t, y)$ and $\xi_{2}(t, y)$, are not independent, but are related by the continuity equation.

We consider first the dynamical constituents of the velocity and vorticity. It follows from Eqs. (5.4), (5.5) that the dynamical constituents $\left.u_{\mathrm{d}}(x, y), v_{\mathrm{d}}(x, y)\right)$ and $\Omega_{\mathrm{d}}(x, y)$ are described by the equations

$$
\begin{aligned}
& \Omega_{\mathrm{d}}(x, y)=\frac{\partial u_{\mathrm{d}}(x, y)}{\partial y}-\frac{\partial v_{\mathrm{d}}(x, y)}{\partial x} \\
& \frac{\partial u_{\mathrm{d}}(x, y)}{\partial x}+\frac{\partial v_{\mathrm{d}}(x, y)}{\partial y}=0 \\
& u_{\mathrm{d}}(x, y) \frac{\partial \Omega_{\mathrm{d}}(x, y)}{\partial x}+v_{\mathrm{d}}(x, y) \frac{\partial \Omega_{\mathrm{d}}(x, y)}{\partial y}-\frac{2}{\operatorname{Re}}\left(\frac{\partial^{2} \Omega_{\mathrm{d}}(x, y)}{\partial x^{2}}+\frac{\partial^{2} \Omega_{\mathrm{d}}(x, y)}{\partial y^{2}}\right)=0 .
\end{aligned}
$$

It is very difficult, if not impossible, to solve these nonlinear equations exactly. Therefore we choose $u_{\mathrm{d}}(x, y)$ in the form of a given function of $y$ with unknown parameters depending on $x$. The shape of this function must depend on whether the outflow from the nozzle is laminar or turbulent. For simplicity, we restrict our consideration to laminar nozzle flow. In this case we can set $u_{\mathrm{d}}(x, y)$ so that, at the nozzle exit section, the boundary layer is close in form to that described by the Blasius equation (see $[14,11]$ )

$$
u_{\mathrm{Bl}}(y)= \begin{cases}F(\eta) & \text { for }|y| \leq 1, \\ 0 & \text { for }|y| \geq 1\end{cases}
$$


where

$$
\eta=\frac{a(1-|y|)}{\delta_{00}}
$$

$\delta_{00}=1 /\left(b_{0} \sqrt{\mathrm{Re}}\right)$ is the relative thickness of the boundary layer at the nozzle exit, $b_{0}$ is determined by the conditions of outflow from the nozzle, $a$ is a parameter which depends on the definition of the boundary layer thickness ${ }^{8}, F(\eta)$ is the derivative with respect to $\eta$ of the Blasius function $f(\eta)$, described by the equation

$$
\frac{d^{3} f}{d \eta^{3}}+\frac{f}{2} \frac{d^{2} f}{d \eta^{2}}=0
$$

with initial conditions $f(0)=0, d f(0) / d \eta=0, d^{2} f(0) / d \eta^{2} \approx 0.332[14,11]$.

Taking account of the entrainment of the ambient fluid, we set the velocity profile close to $(5.10)$ for $x=0$ to the form

$$
u_{\mathrm{d}}(x, y)=\frac{1}{1+\tanh \left(q / \delta_{00}+r_{0}\right)}\left[1-\tanh \left(q \frac{|y|-1}{\delta_{0}(x)}-r(x)\right)\right]
$$

where $\delta_{0}(x)$ and $r(x)$ are unknown functions of $x$, and $\delta_{0}(x)$ is the boundary layer thickness which is equal to $\delta_{00}$ for $x=0, r_{0}=r(0)$. We note that the profile (5.13) was first suggested in [46]; it is similar to that given for the mean velocity in [126-128,132].

The thicknesses of inner and external boundary layers $\left(\delta_{1}(x)\right.$ and $\left.\delta_{2}(x)\right)$ are defined by the relations:

$$
u_{\mathrm{d}}\left(x, 1-\delta_{1}(x)\right)=\alpha, \quad u_{\mathrm{d}}\left(x, 1+\delta_{2}(x)\right)=1-\alpha
$$

where $\alpha$ is a number close to 1 . As follows from (5.13) and (5.14)

$$
q \frac{\delta_{1}(x)}{\delta_{0}(x)}+r(x)=\operatorname{arctanh}(2 \alpha-1), \quad q \frac{\delta_{2}(x)}{\delta_{0}(x)}-r(x)=\operatorname{arctanh}(2 \alpha-1)
$$

\footnotetext{
8 If the boundary layer thickness is defined so that at its boundary the relative velocity is equal to 0.99 , then $a \approx 5$.
} 
Adding Eqs. (5.15) we obtain the relation between $q$ and $\alpha$ :

$$
q=2 \operatorname{arctanh}(2 \alpha-1)
$$

For $\alpha=0.95$ we find $q \approx 3$.

The substitution of (5.16) into (5.15) gives

$$
\frac{\delta_{1}(x)}{\delta_{0}(x)}=\frac{1}{2}-\frac{r(x)}{q}, \quad \frac{\delta_{2}(x)}{\delta_{0}(x)}=\frac{1}{2}+\frac{r(x)}{q} .
$$

The form of the velocity profile determines the so-called shape-factor $H$ [14], which is equal to the ratio between the displacement thickness

$$
\delta^{*}(0)=\frac{1}{\delta_{0}(0)} \int_{0}^{1+\delta_{2}(0)}\left(1-u_{\mathrm{d}}(0, y)\right) d y
$$

and the thickness of momentum loss

$$
\theta(0)=\frac{1}{\delta_{0}(0)} \int_{0}^{1+\delta_{2}(0)} u_{\mathrm{d}}(0, y)\left(1-u_{\mathrm{d}}(0, y)\right) d y
$$

For a turbulent boundary layer, the shape-factor $H$ has to lie in the range 1.4-1.6 [14], whereas for a laminar boundary layer it has to be significantly more. Using the values of parameters calculated above we can calculate $\delta^{*}(0), \theta(0)$ and $H(0)$ for our velocity profile. As a result, we find $\delta^{*}(0) \approx 0.5081, \theta(0) \approx 0.1588$ and $H(0) \approx 3.2$. Thus, our velocity profile does correspond to a laminar boundary layer.

To find the unknown functions in the expression (5.13), we use the conservation laws for the fluxes of momentum and energy. Usually they are derived for the mean values of these fluxes starting from the Reynolds equations [86,21], and therefore contain the so-called turbulent viscosity. We derive them directly starting from Eqs. (5.7)-(5.9) for dynamical constituents. For this we transform Eqs. (5.7)-(5.9) in the following way. Substituting $\Omega_{\mathrm{d}}(x, y)$ from Eq. (5.7) into Eq. (5.9), and taking into account that within the jet's initial part $\partial^{2} u_{\mathrm{d}} / \partial x^{2}$ and $\partial^{2} v_{\mathrm{d}} / \partial x^{2}$ are negligibly small, we obtain

$$
u_{\mathrm{d}}(x, y) \frac{\partial^{2} u_{\mathrm{d}}(x, y)}{\partial x \partial y}+v_{\mathrm{d}}(x, y) \frac{\partial^{2} u_{\mathrm{d}}(x, y)}{\partial y^{2}}-\frac{2}{\operatorname{Re}} \frac{\partial^{3} u_{\mathrm{d}}(x, y)}{\partial y^{3}}=0 .
$$


Taking account of the continuity equation (5.8) we can rewrite Eq. (5.18) as

$$
\frac{\partial}{\partial y}\left(\frac{\partial u_{\mathrm{d}}^{2}(x, y)}{\partial x}+\frac{\partial\left(u_{\mathrm{d}}(x, y) v_{\mathrm{d}}(x, y)\right)}{\partial y}-\frac{2}{\operatorname{Re}} \frac{\partial^{2} u_{\mathrm{d}}(x, y)}{\partial y^{2}}\right)=0
$$

By integrating Eq. (5.19) over y, we obtain the following approximate equation:

$$
\frac{\partial u_{\mathrm{d}}^{2}(x, y)}{\partial x}+\frac{\partial\left(u_{\mathrm{d}}(x, y) v_{\mathrm{d}}(x, y)\right)}{\partial y}-\frac{2}{\operatorname{Re}} \frac{\partial^{2} u_{\mathrm{d}}(x, y)}{\partial y^{2}}=0 .
$$

The conservation law for the dynamical constituent of the momentum flux is found by integrating Eq.(5.20) over $y$ from $-\infty$ to $\infty$, taking into account that $u_{\mathrm{d}}(x, \pm \infty)=0$ and $\partial u_{\mathrm{d}}(x, \pm \infty) / \partial y=0$. We thus obtain

$$
\frac{\partial}{\partial x} \int_{-\infty}^{\infty} u_{\mathrm{d}}^{2}(x, y) d y=0
$$

To derive the conservation law for the dynamical constituent of the energy flux, we multiply Eq. (5.20) by $2 u_{\mathrm{d}}(x, y)$ and transform it to the form

$$
\frac{\partial u_{\mathrm{d}}^{3}}{\partial x}+\frac{\partial\left(u_{\mathrm{d}}^{2} v_{\mathrm{d}}\right)}{\partial y}=\frac{4}{\operatorname{Re}} u_{\mathrm{d}} \frac{\partial^{2} u_{\mathrm{d}}}{\partial y^{2}}
$$

Integrating further Eq. (5.22) over $y$ from $-\infty$ to $\infty$ and taking into account the boundary conditions indicated above we find

$$
\frac{\partial}{\partial x} \int_{-\infty}^{\infty} u_{\mathrm{d}}^{3}(x, y) d y=-\frac{4}{\operatorname{Re}} \int_{-\infty}^{\infty}\left(\frac{\partial u_{\mathrm{d}}(x, y)}{\partial y}\right)^{2} d y
$$

Because $u_{\mathrm{d}}(x, y)$ is an even function of $y$, we obtain from (5.21) and (5.23) the following approximate equations:

$$
\begin{aligned}
& \int_{0}^{\infty} u_{\mathrm{d}}^{2}(x, y) d y=\int_{0}^{\infty} u_{\mathrm{d}}^{2}(0, y) d y \\
& 3 \int_{0}^{\infty} u_{\mathrm{d}}^{2}(x, y) \frac{\partial u_{\mathrm{d}}(x, y)}{\partial x} d y=-\frac{4}{\operatorname{Re}} \int_{0}^{\infty}\left(\frac{\partial u_{\mathrm{d}}(x, y)}{\partial y}\right)^{2} d y
\end{aligned}
$$


Substituting (5.13) into Eq. (5.24) and taking into account that

$$
\int_{0}^{\infty} u_{\mathrm{d}}^{2}(0, y) d y \approx 1
$$

we obtain a relationship between $r(x)$ and $\delta_{0}(x)$ :

$$
\ln \left[2 \cosh \left(\frac{q}{\delta_{0}(x)}+r(x)\right)\right]-\frac{q}{\delta_{0}(x)}+r(x)-\frac{1}{2}\left[1+\tanh \left(\frac{q}{\delta_{0}(x)}+r(x)\right)\right]=0 .
$$

Within the jet part, where

$$
\frac{q}{\delta_{0}(x)} \gg 1
$$

the relationship (5.26) reduces to $2 r(x) \approx 1$, i.e. $r(x) \approx r_{0}=0.5$. It follows from this and (5.19), (5.17) that

$$
\delta_{1}(x) \approx \frac{\delta_{0}(x)}{3}, \quad \delta_{2}(x) \approx \frac{2 \delta_{0}(x)}{3} .
$$

Substituting (5.13) into Eq. (5.25), and taking account of (5.26), we find the differential equation for $\delta_{0}(x)$ :

$$
\begin{aligned}
& \left\{5 \tanh \left(\frac{q}{\delta_{0}(x)}+r_{0}\right)-4-\cosh ^{-2}\left(\frac{q}{\delta_{0}(x)}+r_{0}\right)-\frac{2 q}{\delta_{0}(x)}\left[1-\tanh \left(\frac{q}{\delta_{0}(x)}+r_{0}\right)\right.\right. \\
& \left.\left.+\frac{1}{4}\left(3+\tanh \left(\frac{q}{\delta_{0}(x)}+r_{0}\right)\right) \cosh ^{-2}\left(\frac{q}{\delta_{0}(x)}+r_{0}\right)\right]\right\} \frac{d \delta_{0}(x)}{d x} \\
& =\frac{4 q^{2}}{3 \operatorname{Re} \delta_{0}(x)}\left[1+\tanh \left(\frac{q}{\delta_{0}(x)}+r_{0}\right)\right]\left[1+\tanh \left(\frac{q}{\delta_{0}(x)}+r_{0}\right)+\cosh ^{-2}\left(\frac{q}{\delta_{0}(x)}+r_{0}\right)\right] .
\end{aligned}
$$

A solution of this equation can be found analytically only for the condition (5.27). In this case Eq. (5.29) becomes

$$
\frac{d \delta_{0}(x)}{d x}=\frac{16 q^{2}}{3 \operatorname{Re} \delta_{0}(x)}
$$

It follows from Eq. (5.30) that

$$
\delta_{0}(x)=\sqrt{\delta_{00}^{2}+2 k x}, \quad \frac{d \delta_{0}(x)}{d x}=\frac{k}{\delta_{0}(x)},
$$


where $k=16 q^{2} /(3 \operatorname{Re})$.

We note that the dependence $\delta_{0}(x)$ found here from the Navier-Stokes equations differs from that found from the Reynolds equations [86,21] and containing the turbulent viscosity $\nu_{\mathrm{t}}$. Since, by Prandtl's hypothesis [13], $\nu_{\mathrm{t}}$ is proportional to the boundary layer thickness $\delta(x)$, the dependence $\delta(x)$ was found to be linear.

The expressions for $v_{\mathrm{d}}(x, y)$ and $\Omega_{\mathrm{d}}(x, y)$ can be found by exact solution of Eqs. (5.7), (5.8). As a result, we obtain

$$
\begin{aligned}
& v_{\mathrm{d}}(x, y)=-\frac{16 q \operatorname{sign} y}{3 \operatorname{Re} \delta_{0}(x)\left(1+\tanh \left(q / \delta_{00}+r_{0}\right)\right)}\left[\frac{q(|y|-1)}{\delta_{0}(x)} \tanh \left(\frac{q(|y|-1)}{\delta_{0}(x)}-r_{0}\right)\right. \\
& \left.-\frac{q}{\delta_{0}(x)} \tanh \left(\frac{q}{\delta_{0}(x)}+r_{0}\right)-\ln \frac{\cosh \left(q(|y|-1) / \delta_{0}(x)-r_{0}\right)}{\cosh \left(q / \delta_{0}(x)+r_{0}\right)}\right], \\
& \Omega_{\mathrm{d}}(x, y)=-\frac{q \operatorname{sign} y}{\delta_{0}(x)\left(1+\tanh \left(q / \delta_{00}+r_{0}\right)\right)}\left\{\left(1+\frac{256 q^{4}(|y|-1)^{2}}{9 \delta_{0}^{4}(x) \operatorname{Re}^{2}}\right) \cosh ^{-2}\left(\frac{q(|y|-1)}{\delta_{0}(x)}-r_{0}\right)\right. \\
& -\frac{256 q^{2}}{9 \delta_{0}^{2}(x) \operatorname{Re}^{2}}\left[\frac{q^{2}}{\delta_{0}^{2}(x)} \cosh { }^{-2}\left(\frac{q}{\delta_{0}(x)}+r_{0}\right)-\frac{q}{\delta_{0}(x)}\left((|y|-1) \tanh \left(\frac{q(|y|-1)}{\delta_{0}(x)}-r_{0}\right)\right.\right. \\
& \left.\left.\left.-\tanh \left(\frac{q}{\delta_{0}(x)}+r_{0}\right)\right)+\ln \frac{\cosh \left(q(|y|-1) / \delta_{0}(x)-r_{0}\right)}{\cosh \left(q / \delta_{0}(x)+r_{0}\right)}\right]\right\} .
\end{aligned}
$$

For the condition (5.27), from (5.32)and (5.33) we find the following approximate asymptotic expressions for $v_{\mathrm{d}}$ and $\Omega_{\mathrm{d}}$ :

$$
v_{\mathrm{d}}(x, \pm \infty) \approx \mp \frac{16 q r_{0}}{3 \delta_{0}(x) \operatorname{Re}}, \quad \Omega_{\mathrm{d}}(x, \pm \infty) \approx \mp \frac{256 q^{3} r_{0}}{9 \delta_{0}^{3}(x) \operatorname{Re}^{2}}
$$

Fig. 23 shows plots of $u_{\mathrm{d}}(x, y), v_{\mathrm{d}}(x, y), \Omega_{\mathrm{d}}(x, y)$ versus $y$ for $b_{0}=0.1, q=3, r_{0}=0.5$, $\operatorname{Re}=25000, x=0$ and $x=8$. We see that for all values of $y$, except for narrow intervals near $y= \pm 1, u_{\mathrm{d}}(x, y), v_{\mathrm{d}}(x, y)$ and $\Omega_{\mathrm{d}}(x, y)$ are nearly constant. The constant transverse velocity component for $|y|>1$ directed towards the jet axis accounts for the entrainment of ambient fluid with the jet flow. 
It should be emphasized that the results obtained here concern only the dynamical constituents of the velocity and vorticity. Stochastic constituents greatly influence the thickness of the boundary layer, its dependence on the distance from the nozzle, and values of the mean velocities (see below).

Substituting further

$$
\begin{aligned}
& U(t, x, y)=u_{\mathrm{d}}(x, y)+\frac{\partial \psi(t, x, y)}{\partial y}, \quad V(t, x, y)=v_{\mathrm{d}}(x, y)-\frac{\partial \psi(t, x, y)}{\partial x}, \\
& \tilde{\Omega}(t, x, y)=\Omega_{\mathrm{d}}(x, y)+\Omega(t, x, y)
\end{aligned}
$$

into Eqs. (5.4), (5.5) and taking into account (5.7)-(5.9), we find the equations for the stochastic constituents $\psi(t, x, y)$ and $\Omega(t, x, y)$, which we write in the form

$$
\begin{aligned}
& \Omega-\Delta \psi=0, \\
& \frac{\partial \Omega}{\partial t}+u_{\mathrm{d}}(x, y) \frac{\partial \Omega}{\partial x}+v_{\mathrm{d}}(x, y) \frac{\partial \Omega}{\partial y}-\Omega_{\mathrm{d} y}(x, y) \frac{\partial \psi}{\partial x}+\Omega_{\mathrm{d} x}(x, y) \frac{\partial \psi}{\partial y}-\frac{2}{\operatorname{Re}} \Delta \Omega \\
& =\frac{\partial \psi}{\partial x} \frac{\partial \Omega}{\partial y}-\frac{\partial \psi}{\partial y} \frac{\partial \Omega}{\partial x}
\end{aligned}
$$

where

$$
\Omega_{\mathrm{d} x}(x, y)=\frac{\partial \Omega_{\mathrm{d}}(x, y)}{\partial x}, \quad \Omega_{\mathrm{d} y}(x, y)=\frac{\partial \Omega_{\mathrm{d}}(x, y)}{\partial y}
$$

According to (5.6) the boundary conditions for Eqs. (5.36), (5.37) are

$$
\left.\frac{\partial \psi}{\partial y}\right|_{x=0}=\xi_{1}(t, y),\left.\quad \frac{\partial \psi}{\partial x}\right|_{x=0}=-\xi_{2}(t, y) .
$$

\section{The derivation of truncated equations for the amplitude of stochastic con- stituents}

To describe the development of turbulence, we can assume that the right-hand side of Eq. (5.37) is of the order of a small parameter $\epsilon$. In this case Eqs. (5.36), (5.37) can be solved approximately by a method similar to the Krylov-Bogolyubov method for spatially extended systems [131]. We therefore seek a solution in the form of a series in $\epsilon$ : 


$$
\begin{aligned}
& \Omega(t, x, y)=\Omega_{0}(t, x, y)+\epsilon r_{1}(t, x, y)+\epsilon^{2} r_{2}(t, x, y)+\ldots \\
& \psi(t, x, y)=\psi_{0}(t, x, y)+\epsilon s_{1}(t, x, y)+\epsilon^{2} s_{2}(t, x, y)+\ldots \\
& u(t, x, y)=\frac{\partial \psi(t, x, y)}{\partial y}=u_{0}(t, x, y)+\epsilon q_{1}(t, x, y)+\epsilon^{2} q_{2}(t, x, y)+\ldots
\end{aligned}
$$

where $\Omega_{0}(t, x, y)$ and $\psi_{0}(t, x, y)$ are generative solutions of Eqs. (5.36), (5.37), $u_{0}(t, x, y)=$ $\partial \psi_{0}(t, x, y) / \partial y ; r_{1}(t, x, y), r_{2}(t, x, y), \ldots, s_{1}(t, x, y), s_{2}(t, x, y), \ldots$ are unknown functions, and $q_{1}(t, x, y)=\partial s_{1}(t, x, y) / \partial y, q_{2}(t, x, y)=\partial s_{2}(t, x, y) / \partial y, \ldots$

It should be emphasized that because of the quadratic nonlinearity the contribution of nonlinear terms into turbulent processes can be estimated only by using the second approximation of the Krylov-Bogolyubov method. Thus in the expansion (6.1) we have to retain the terms up to the second order with respect to $\epsilon$.

\subsection{Generative solutions}

Putting the right-hand side of Eq. (5.37) to zero and eliminating the stochastic constituent of vorticity, we obtain the generative equation for the stochastic constituent of the stream function:

$$
\begin{aligned}
& \frac{\partial \Delta \psi_{0}}{\partial t}+u_{\mathrm{d}}(x, y) \frac{\partial \Delta \psi_{0}}{\partial x}+v_{\mathrm{d}}(x, y) \frac{\partial \Delta \psi_{0}}{\partial y} \\
& -\Omega_{\mathrm{d} y}(x, y) \frac{\partial \psi_{0}}{\partial x}+\Omega_{\mathrm{d} x}(x, y) \frac{\partial \psi_{0}}{\partial y}-\frac{2}{\operatorname{Re}} \Delta \Delta \psi_{0}=0 .
\end{aligned}
$$

It should be noted that finding the generative solution is similar to the well known problem of the linear instability of a jet flow. During the last three decades, this problem was studied primarily by Crighton and Gaster [126,132], Michalke [128] and Plaschko [127]. In these works a profile of the mean flow velocity for a circular jet was given, and the problem was solved approximately, mainly within the framework of linearized Euler equations. Because the coefficients of these equations depend on the coordinates, an exact analytic solution could not be found. Numerical calculations performed by these authors are in qualitative agreement with experimental data.

Here we find the generative solution for a plane jet based on the linearized Navier-Stokes 
equation (6.2) and using the dynamical constituents of velocity and vorticity calculated above. We emphasize that viscosity should be taken into account, because all terms in Eq. (6.2) are of the same order over the region of the boundary layer. We chose a plane jet, rather than a circular one, by virtue of the same reasoning as in [92]: its simple geometry and boundary conditions.

We seek a partial solution of Eq. (6.2) in the form of a sum of running waves of frequency $\mathrm{S}$ with a slowly varying complex wave number $Q(\mathrm{~S}, x)$ :

$$
\psi_{0}(t, x, y)=\frac{1}{2 \pi} \int_{-\infty}^{\infty} f^{(\mathrm{S})}(x, y) \exp \left[i\left(\mathrm{~S} t-\int_{0}^{x} Q(\mathrm{~S}, x) d x\right)\right] d \mathrm{~S} .
$$

Taking into account that the jet diverges slowly, we can represent the function $f^{(\mathrm{S})}(x, y)$ and the wave number $Q(\mathrm{~S}, x)$ as series in a conditional small parameter $\mu \sim 1 / \sqrt{\operatorname{Re}}$ :

$f^{(\mathrm{S})}(x, y)=f_{0}(\mathrm{~S}, x, y)+\mu f_{1}(\mathrm{~S}, x, y)+\ldots, \quad Q(\mathrm{~S}, x)=Q_{0}(\mathrm{~S}, x)+\mu Q_{1}(\mathrm{~S}, x)+\ldots$,

where $f_{0}(\mathrm{~S}, x, y), f_{1}(\mathrm{~S}, x, y), \ldots$ are unknown functions vanishing, along with their derivatives, at $y= \pm \infty$.

Substituting (6.3), in view of (6.4), into Eq. (6.2) and retaining only terms containing first derivatives with respect to $x$ we obtain the following equations for $f_{0}(\mathrm{~S}, x, y)$ and $f_{1}(\mathrm{~S}, x, y)$ :

$$
\begin{aligned}
& L_{0}\left(Q_{0}\right) f_{0}=0, \\
& L_{0}\left(Q_{0}\right) f_{1}=i Q_{1} L_{1}\left(Q_{0}\right) f_{0}-L_{2}\left(Q_{0}\right) f_{0},
\end{aligned}
$$

where

$$
\begin{aligned}
& L_{0}\left(Q_{0}\right)=i\left(\mathrm{~S}-u_{\mathrm{d}}(x, y) Q_{0}\right)\left(\frac{\partial^{2}}{\partial y^{2}}-Q_{0}^{2}\right)+v_{\mathrm{d}}(x, y)\left(\frac{\partial^{3}}{\partial y^{3}}-Q_{0}^{2} \frac{\partial}{\partial y}\right) \\
& +i Q_{0} \Omega_{\mathrm{d} y}(x, y)+\Omega_{\mathrm{d} x}(x, y) \frac{\partial}{\partial y}-\frac{2}{\operatorname{Re}}\left(\frac{\partial^{4}}{\partial y^{4}}-2 Q_{0}^{2} \frac{\partial^{2}}{\partial y^{2}}+Q_{0}^{4}\right) \\
& L_{1}\left(Q_{0}\right)=u_{\mathrm{d}}(x, y)\left(\frac{\partial^{2}}{\partial y^{2}}-3 Q_{0}^{2}\right)+2 \mathrm{~S} Q_{0}-2 i Q_{0} v_{\mathrm{d}}(x, y) \frac{\partial}{\partial y} \\
& -\Omega_{\mathrm{d} y}(x, y)+\frac{8 i Q_{0}}{\operatorname{Re}}\left(\frac{\partial^{2}}{\partial y^{2}}-Q_{0}^{2}\right)
\end{aligned}
$$




$$
\begin{gathered}
L_{2}\left(Q_{0}\right)=\mathrm{S}\left(2 Q_{0} \frac{\partial}{\partial x}+\frac{\partial Q_{0}}{\partial x}\right)+u_{\mathrm{d}}(x, y)\left[\frac{\partial^{3}}{\partial x \partial y^{2}}-3 Q_{0}\left(Q_{0} \frac{\partial}{\partial x}+\frac{\partial Q_{0}}{\partial x}\right)\right]-i v_{\mathrm{d}}(x, y) \\
\times\left(2 Q_{0} \frac{\partial^{2}}{\partial x \partial y}+\frac{\partial Q_{0}}{\partial x} \frac{\partial}{\partial y}\right)-\Omega_{\mathrm{d} y}(x, y) \frac{\partial}{\partial x}+\frac{4 i}{\operatorname{Re}}\left[2 Q_{0} \frac{\partial^{3}}{\partial x \partial y^{2}}+\frac{\partial Q_{0}}{\partial x} \frac{\partial^{2}}{\partial y^{2}}\right. \\
\left.-Q_{0}^{2}\left(2 Q_{0} \frac{\partial}{\partial x}+3 \frac{\partial Q_{0}}{\partial x}\right)\right]
\end{gathered}
$$

Eq. (6.5), with the boundary conditions for function $f_{0}$ and its derivatives so as to be vanishing at $y= \pm \infty$, describes a non-self-adjoint boundary-value problem, where $Q_{0}$ plays the role of an eigenvalue. Similar boundary-value problems, but on a finite interval, were studied by Keldysh [129]. Consistent with Fredholm's well known theorem [130] about linear boundary-value problems described by an inhomogeneous equation, Eq. (6.6) has a nontrivial solution only if

$$
i Q_{1} \int_{-\infty}^{\infty} \bar{\chi}(\mathrm{S}, x, y) L_{1}\left(Q_{0}\right) f_{0}(\mathrm{~S}, x, y) d y-\int_{-\infty}^{\infty} \bar{\chi}(\mathrm{S}, x, y) L_{2}\left(Q_{0}\right) f_{0}(\mathrm{~S}, x, y) d y=0
$$

where $\bar{\chi}(\mathrm{S}, x, y)$ is a complex conjugate eigenfunction of the adjoint boundary-value problem described by the equation:

$$
\begin{aligned}
& i\left(\frac{\partial^{2}}{\partial y^{2}}-Q_{0}^{2}\right)\left[\left(\mathrm{S}-u_{\mathrm{d}}(x, y) Q_{0}\right) \bar{\chi}\right]-\left(\frac{\partial^{3}}{\partial y^{3}}-Q_{0}^{2} \frac{\partial}{\partial y}\right)\left(v_{\mathrm{d}}(x, y) \bar{\chi}\right) \\
& +i Q_{0} \Omega_{\mathrm{d} y}(x, y) \bar{\chi}-\frac{\partial\left(\Omega_{\mathrm{d} x}(x, y) \bar{\chi}\right)}{\partial y}-\frac{2}{\lambda^{2}}\left(\frac{\partial^{4} \bar{\chi}}{\partial y^{4}}-2 Q_{0}^{2} \frac{\partial^{2} \bar{\chi}}{\partial y^{2}}+Q_{0}^{4} \bar{\chi}\right)=0 .
\end{aligned}
$$

The over-bar in Eq. (6.10) implies the operation of complex conjugation. The condition (6.10) allows us to find the small correction $Q_{1}(\mathrm{~S}, x)$ to the eigenvalue $Q_{0}(\mathrm{~S}, x)$.

Over the region of $|y| \leq y_{1}(x)$ (region $\mathrm{I}$ ), where $y_{1}(x)$ is the internal boundary of the boundary layer, $u_{\mathrm{d}}(x, y) \approx 1, v_{\mathrm{d}}(x, y) \approx 0, \Omega_{\mathrm{d} x}(x, y) \approx 0$ and $\Omega_{\mathrm{d} y}(x, y) \approx 0$. For this region the general solution of Eqs. (6.5) and (6.11) is

$$
\begin{aligned}
& f_{0}(y)=A_{1} \sinh \left(B_{11} y\right)+A_{2} \sinh \left(B_{12} y\right)+A_{3} \cosh \left(B_{11} y\right)+A_{4} \cosh \left(B_{12} y\right), \\
& \bar{\chi}(y)=\tilde{A}_{1} \sinh \left(B_{11} y\right)+\tilde{A}_{2} \sinh \left(B_{12} y\right)+\tilde{A}_{3} \cosh \left(B_{11} y\right)+\tilde{A}_{4} \cosh \left(B_{12} y\right),
\end{aligned}
$$


where

$$
B_{11}=Q_{0}, \quad B_{12}=\sqrt{Q_{0}^{2}+\frac{i\left(\mathrm{~S}-Q_{0}\right) \operatorname{Re}}{2}}
$$

are the roots of the characteristic equation corresponding to Eqs. (6.5) and (6.11), and $A_{1}, A_{2}, A_{3}, A_{4}, \tilde{A}_{1}, \tilde{A}_{2}, \tilde{A}_{3}$ and $\tilde{A}_{4}$ are arbitrary constants.

Any arbitrary disturbance can be represented as a linear combination of even and odd constituents. We consider the case of odd disturbances. In this case we can solve Eqs. (6.5) and (6.11) only for positive values of $y$, seeking a solution of these equations in the form

$$
f_{0}(y)=A_{1} f_{01}(y)+A_{2} f_{02}(y), \quad \bar{\chi}_{0}(y)=\tilde{A}_{1} \bar{\chi}_{01}(y)+\tilde{A}_{2} \bar{\chi}_{02}(y)
$$

where, for $|y| \leq y_{1}(x)$, the functions $f_{01}(y), \bar{\chi}_{1}(y)$ transform to $\sinh \left(B_{11} y\right)$, and $f_{02}(y)$, $\bar{\chi}_{2}(y)$ transform to $\sinh \left(B_{12} y\right)$. It follows that $f_{01}(y), \bar{\chi}_{01}(y), f_{02}(y)$ and $\bar{\chi}_{02}(y)$ must satisfy the following initial conditions:

$$
\begin{aligned}
& f_{01}(0)=\bar{\chi}_{01}(0)=0,\left.\quad \frac{\partial f_{01}}{\partial y}\right|_{y=0}=\left.\frac{\partial \bar{\chi}_{01}}{\partial y}\right|_{y=0}=B_{11}, \\
& \left.\frac{\partial^{2} f_{01}}{\partial y^{2}}\right|_{y=0}=\left.\frac{\partial^{2} \bar{\chi}_{01}}{\partial y^{2}}\right|_{y=0}=0,\left.\quad \frac{\partial^{3} f_{01}}{\partial y^{3}}\right|_{y=0}=\left.\frac{\partial^{3} \bar{\chi}_{01}}{\partial y^{3}}\right|_{y=0}=B_{11}^{3}, \\
& f_{02}(0)=\bar{\chi}_{02}(0)=0,\left.\quad \frac{\partial f_{02}}{\partial y}\right|_{y=0}=\left.\frac{\partial \bar{\chi}_{02}}{\partial y}\right|_{y=0}=B_{12}, \\
& \left.\frac{\partial^{2} f_{02}}{\partial y^{2}}\right|_{y=0}=\left.\frac{\partial^{2} \bar{\chi}_{02}}{\partial y^{2}}\right|_{y=0}=0,\left.\quad \frac{\partial^{3} f_{02}}{\partial y^{3}}\right|_{y=0}=\left.\frac{\partial^{3} \bar{\chi}_{02}}{\partial y^{3}}\right|_{y=0}=B_{12}^{3} .
\end{aligned}
$$

Over the region of $|y| \geq y_{2}(x)$ (region II), where $y_{2}(x)$ is the external boundary of the boundary layer, $u_{\mathrm{d}}(x, y) \approx 0, v_{\mathrm{d}}(x, y) \approx v_{\mathrm{d}}(x, \infty), \Omega_{\mathrm{d} x}(x, y) \approx \Omega_{\mathrm{d} x}(x, \infty)$ and $\Omega_{\mathrm{d} y}(x, y) \approx 0$. For this region the solutions of Eqs. (6.5) and (6.11) must behave as partial solutions of the equations 


$$
\begin{aligned}
& i \mathrm{~S}\left(\frac{\partial^{2} f_{0}}{\partial y^{2}}-Q_{0}^{2} f_{0}\right)+v_{\mathrm{d}}(x, \infty)\left(\frac{\partial^{3} f_{0}}{\partial y^{3}}-Q_{0}^{2} \frac{\partial f_{0}}{\partial y}\right) \\
& +\Omega_{\mathrm{d} x}(x, \infty) \frac{\partial f_{0}}{\partial y}-\frac{2}{\operatorname{Re}}\left(\frac{\partial^{4} f_{0}}{\partial y^{4}}-2 Q_{0}^{2} \frac{\partial^{2} f_{0}}{\partial y^{2}}+Q_{0}^{4} f_{0}\right)=0 \\
& i \mathrm{~S}\left(\frac{\partial^{2} \bar{\chi}_{0}}{\partial y^{2}}-Q_{0}^{2} \bar{\chi}_{0}\right)-v_{\mathrm{d}}(x, \infty)\left(\frac{\partial^{3} \bar{\chi}_{0}}{\partial y^{3}}-Q_{0}^{2} \frac{\partial \bar{\chi}_{0}}{\partial y}\right) \\
& -\Omega_{\mathrm{d} x}(x, \infty) \frac{\partial \bar{\chi}_{0}}{\partial y}-\frac{2}{\operatorname{Re}}\left(\frac{\partial^{4} \bar{\chi}_{0}}{\partial y^{4}}-2 Q_{0}^{2} \frac{\partial^{2} \bar{\chi}_{0}}{\partial y^{2}}+Q_{0}^{4} \bar{\chi}_{0}\right)=0
\end{aligned}
$$

satisfying the condition of vanishing at $y=\infty$. Such partial solutions can be written as

$$
\begin{aligned}
& f_{0}(y)=C_{21} \exp \left[B_{21}\left(y-y_{2}(x)\right)\right]+C_{22} \exp \left[B_{22}\left(y-y_{2}(x)\right)\right], \\
& \bar{\chi}_{0}(y)=\tilde{C}_{21} \exp \left[\tilde{B}_{21}\left(y-y_{2}(x)\right)\right]+\tilde{C}_{22} \exp \left[\tilde{B}_{22}\left(y-y_{2}(x)\right)\right],
\end{aligned}
$$

where $C_{21}, C_{22}, \tilde{C}_{21}$ and $\tilde{C}_{22}$ are arbitrary constants, and $B_{21}, B_{22}, \tilde{B}_{21}$ and $\tilde{B}_{22}$ are roots of the characteristic equations corresponding to Eqs. (6.16) with negative real parts. The characteristic equations for region II take the form

$$
\begin{aligned}
& B^{4}-a_{10} B^{3}-a_{20} B^{2}-a_{30} B+a_{40}=0, \\
& \tilde{B}^{4}+a_{10} \tilde{B}^{3}-a_{20} \tilde{B}^{2}+a_{30} \tilde{B}+a_{40}=0,
\end{aligned}
$$

where

$$
\begin{aligned}
& a_{10}= \pm \frac{v_{\mathrm{d}}(x, \infty) \operatorname{Re}}{2}, a_{20}=2 Q_{0}^{2}+\frac{i \mathrm{SRe}}{2} \\
& a_{30}= \pm \frac{\left(\Omega_{\mathrm{d} x}(x, \infty)-Q_{0}^{2} v_{\mathrm{d}}(x, \infty)\right) \operatorname{Re}}{2}, \quad a_{40}=Q_{0}^{4}+\frac{i \mathrm{~S} Q_{0}^{2} \operatorname{Re}}{2}
\end{aligned}
$$

the signs ' + ' and ' - ' correspond to $y>0$ and $y<0$, respectively. It follows from (6.18) and (6.19) that $j$ th root of Eq. (6.18) for $y<0$ is equal to $j$ th root for $y>0$ of opposite sign. That is why we need consider only $y>0$.

In view of (6.19), the first equation of (6.18) can be conveniently rewritten as

$$
\left[B^{2}-\frac{v_{\mathrm{d}}(x, \infty) \operatorname{Re}}{2} B-\left(Q_{0}^{2}+\frac{i \mathrm{SRe}}{2}\right)\right]\left(B^{2}-Q_{0}^{2}\right)=\frac{\Omega_{\mathrm{d} x}(x, \infty) \operatorname{Re}}{2} B
$$


It can be shown that the right-hand side of Eq. (6.20) is small. Therefore the roots of Eq. (6.20) with negative real parts are approximately equal to

$$
B_{21}=-Q_{0}+\Delta B_{1}, \quad B_{22}=B_{220}+\Delta B_{2}
$$

where

$$
\begin{aligned}
& B_{220}=\frac{v_{\mathrm{d}}(x, \infty) \operatorname{Re}}{4}\left(1+\sqrt{1+\frac{8 i \mathrm{~S}}{v_{\mathrm{d}}^{2}(x, \infty) \operatorname{Re}}+\frac{16 Q_{0}^{2}}{v_{\mathrm{d}}^{2}(x, \infty) \operatorname{Re}^{2}}}\right) \\
& \Delta B_{1}=-\frac{\Omega_{\mathrm{d} x}(x, \infty)}{2\left(i \mathrm{~S}-v_{\mathrm{d}}(x, \infty) Q_{0}\right)}, \quad \Delta B_{2}=\frac{\Omega_{\mathrm{d} x}(x, \infty) B_{220}}{v_{\mathrm{d}}(x, \infty)\left(B_{220}^{2}+Q_{0}^{2}\right)+i \mathrm{~S} B_{220}} .
\end{aligned}
$$

Equating (6.14) to (6.17) at the point $y=y_{2}(x)$, we find the following boundary conditions:

$$
\begin{aligned}
& A_{1} f_{01}\left(y_{2}\right)+A_{2} f_{02}\left(y_{2}\right)=C_{21}+C_{22}, \quad A_{1} f_{11}\left(y_{2}\right)+A_{2} f_{12}\left(y_{2}\right)=B_{21} C_{21}+B_{22} C_{22}, \\
& A_{1} f_{21}\left(y_{2}\right)+A_{2} f_{22}\left(y_{2}\right)=B_{21}^{2} C_{21}+B_{22}^{2} C_{22}, \quad A_{1} f_{31}\left(y_{2}\right)+A_{2} f_{32}\left(y_{2}\right)=B_{21}^{3} C_{21}+B_{22}^{3} C_{22}, \\
& \tilde{A}_{1} \bar{\chi}_{01}\left(y_{2}\right)+\tilde{A}_{2} \bar{\chi}_{02}\left(y_{2}\right)=\tilde{C}_{21}+\tilde{C}_{22}, \quad \tilde{A}_{1} \bar{\chi}_{11}\left(y_{2}\right)+\tilde{A}_{2} \bar{\chi}_{12}\left(y_{2}\right)=\tilde{B}_{21} \tilde{C}_{21}+\tilde{B}_{22} \tilde{C}_{22}, \\
& \tilde{A}_{1} \bar{\chi}_{21}\left(y_{2}\right)+\tilde{A}_{2} \bar{\chi}_{22}\left(y_{2}\right)=\tilde{B}_{21 a}^{2} \tilde{C}_{21}+\tilde{B}_{22 a}^{2} \tilde{C}_{22}, \quad \tilde{A}_{1} \bar{\chi}_{31}\left(y_{2}\right)+\tilde{A}_{2} \bar{\chi}_{32}\left(y_{2}\right)=\tilde{B}_{21}^{3} \tilde{C}_{21}+\tilde{B}_{22}^{3} \tilde{C}_{22},
\end{aligned}
$$

where

$$
\begin{aligned}
& f_{11}\left(y_{2}\right)=\left.\frac{\partial f_{01}}{\partial y}\right|_{y=y_{2}}, \quad f_{12}\left(y_{2}\right)=\left.\frac{\partial f_{02}}{\partial y}\right|_{y=y_{2}}, \quad f_{21}\left(y_{2}\right)=\left.\frac{\partial^{2} f_{01}}{\partial y^{2}}\right|_{y=y_{2}}, \\
& f_{22}\left(y_{2}\right)=\left.\frac{\partial^{2} f_{02}}{\partial y^{2}}\right|_{y=y_{2}}, \quad f_{31}\left(y_{2}\right)=\left.\frac{\partial^{3} f_{01}}{\partial y^{3}}\right|_{y=y_{2}}, \quad f_{32}\left(y_{2}\right)=\left.\frac{\partial^{3} f_{02}}{\partial y^{3}}\right|_{y=y_{2}} . \\
& \bar{\chi}_{11}\left(y_{2}\right)=\left.\frac{\partial \bar{\chi}_{01}}{\partial y}\right|_{y=y_{2}}, \quad \bar{\chi}_{12}\left(y_{2}\right)=\left.\frac{\partial \bar{\chi}_{02}}{\partial y}\right|_{y=y_{2}}, \quad \bar{\chi}_{21}\left(y_{2}\right)=\left.\frac{\partial^{2} \bar{\chi}_{01}}{\partial y^{2}}\right|_{y=y_{2}}, \\
& \bar{\chi}_{22}\left(y_{2}\right)=\left.\frac{\partial^{2} \bar{\chi}_{02}}{\partial y^{2}}\right|_{y=y_{2}}, \quad \bar{\chi}_{31}\left(y_{2}\right)=\left.\frac{\partial^{3} \bar{\chi}_{01}}{\partial y^{3}}\right|_{y=y_{2}}, \quad \bar{\chi}_{32}\left(y_{2}\right)=\left.\frac{\partial^{3} \bar{\chi}_{02}}{\partial y^{3}}\right|_{y=y_{2}} .
\end{aligned}
$$

The eigenvalues of $Q_{0}$ for the basic boundary-value problem must satisfy the requirement 
that the determinant

$$
D\left(Q_{0}\right)=\left|\begin{array}{llll}
f_{01}\left(y_{2}\right) & f_{02}\left(y_{2}\right) & 1 & 1 \\
f_{11}\left(y_{2}\right) & f_{12}\left(y_{2}\right) & B_{21} & B_{22} \\
f_{21}\left(y_{2}\right) & f_{22}\left(y_{2}\right) & B_{21}^{2} & B_{22}^{2} \\
f_{31}\left(y_{2}\right) & f_{32}\left(y_{2}\right) & B_{21}^{3} & B_{22}^{3}
\end{array}\right|
$$

be equal to zero. It can be shown that the eigenvalues for the adjoint boundary-value problem coincide with those for the basic boundary-value problem.

The expression for $D\left(Q_{0}\right)$ can be written as

$$
\begin{aligned}
& D\left(Q_{0}\right)=q_{23}\left(y_{2}\right)-\left(B_{21}+B_{22}\right) q_{13}\left(y_{2}\right)+\left(B_{21}^{2}+B_{22}^{2}\right) q_{12}\left(y_{2}\right) \\
& +B_{21} B_{22}\left(q_{12}\left(y_{2}\right)+q_{03}\left(y_{2}\right)-\left(B_{21}+B_{22}\right) q_{02}\left(y_{2}\right)\right)+B_{21}^{2} B_{22}^{2} q_{01}\left(y_{2}\right),
\end{aligned}
$$

where

$$
\begin{array}{ll}
q_{01}(y)=f_{01}(y) f_{12}(y)-f_{11}(y) f_{02}(y), & q_{02}(y)=f_{01}(y) f_{22}(y)-f_{21}(y) f_{02}(y), \\
q_{03}(y)=f_{01}(y) f_{32}(y)-f_{31}(y) f_{02}(y), & q_{12}(y)=f_{11}(y) f_{22}(y)-f_{21}(y) f_{12}(y), \\
q_{13}(y)=f_{11}(y) f_{32}(y)-f_{31}(y) f_{12}(y), & q_{23}(y)=f_{21}(y) f_{32}(y)-f_{31}(y) f_{22}(y) .
\end{array}
$$

A direct numerical calculation of $D\left(Q_{0}\right)$, starting from Eqs. (6.5) and (6.25), gives random values on account of the need to subtract large numbers of the same order. Therefore, instead of Eq. (6.5), we solve the equations for $q_{i j}(y)$ which follow from (6.5), (6.27). They are 


$$
\begin{aligned}
& \frac{\partial q_{01}}{\partial y}=q_{02}(y), \quad \frac{\partial q_{02}}{\partial y}=q_{03}(y)+q_{12}(y), \quad \frac{\partial q_{12}}{\partial y}=q_{13}(y) \\
& \frac{\partial q_{03}}{\partial y}=q_{13}(y)+\left(2 Q_{0}^{2}+\frac{i\left(\mathrm{~S}-u_{\mathrm{d}}(x, y) Q_{0}\right) \operatorname{Re}}{2}\right) q_{02}(y) \\
& +\frac{\operatorname{Re}}{2}\left[v_{\mathrm{d}}(x, y)\left[q_{03}(y)-Q_{0}^{2} q_{01}(y)\right]+\Omega_{\mathrm{d} x}(x, y) q_{01}(y)\right] \\
& \frac{\partial q_{13}}{\partial y}=q_{23}(y)+\left(2 Q_{0}^{2}+\frac{i\left(\mathrm{~S}-u_{\mathrm{d}}(x, y) Q_{0}\right) \operatorname{Re}}{2}\right) q_{12}(y) \\
& +Q_{0}^{4} q_{01}(y)+\frac{\operatorname{Re}}{2}\left[\left[i Q_{0}^{2}\left(\mathrm{~S}-u_{\mathrm{d}}(x, y) Q_{0}\right)-i Q_{0} \Omega_{\mathrm{d} y}(x, y)\right] q_{01}(y)+v_{\mathrm{d}}(x, y) q_{13}(y)\right] \\
& \frac{\partial q_{23}}{\partial y}=Q_{0}^{4} q_{02}(y)+\frac{\operatorname{Re}}{2}\left[\left[i Q_{0}^{2}\left(\mathrm{~S}-u_{\mathrm{d}}(x, y) Q_{0}\right)-i Q_{0} \Omega_{\mathrm{d} y}(x, y)\right] q_{02}(y)\right. \\
& \left.+v_{\mathrm{d}}(x, y)\left(q_{23}(y)+Q_{0}^{2} q_{12}(y)\right)-\Omega_{\mathrm{d} x}(x, y) q_{12}(y)\right] .
\end{aligned}
$$

Solving Eqs. (6.28) with initial conditions

$$
q_{01}(0)=q_{02}(0)=q_{03}(0)=q_{12}(0)=q_{23}(0)=0, \quad q_{13}(0)=B_{11} B_{12}\left(B_{12}^{2}-B_{11}^{2}\right),
$$

and substituting the solution found for $y=y_{2}$ into (6.25), we calculate $D\left(Q_{0}\right)$. By varying $Q_{0}$ until $D\left(Q_{0}\right)$ becomes equal to zero, we find the eigenvalues of $Q_{0}$. The real part of the eigenvalue of $Q_{0}$ gives the real wave number $K_{0}$, whereas its imaginary part gives the wave gain factor $\Gamma_{0}$. The dependences of the gain factor $\Gamma_{0}$ and wave phase velocity $v_{\mathrm{ph} 0}=\mathrm{S} / K_{0}$ on the Strouhal number St are given for $\mathrm{Re}=25000, b_{0}=0.1$ and a number values of $x$ in Fig. 24 a and $b$.

To estimate the influence of the Reynolds number and the thickness of the boundary layer at the nozzle exit, we have calculated the eigenvalues of $Q_{0}$ for $x=0$ in two cases: $\operatorname{Re}=100000, b_{0}=0.05$ and $\operatorname{Re}=100000, b_{0}=0.02$. In the first case the thickness of the boundary layer at the nozzle exit is the same as in Fig. 24, and in the second case it is considerably larger. The results are shown in Fig. 25. We see that, for the same thickness of boundary layer at the nozzle exit the results depend only weakly on the Reynolds number, whereas the thickness of the boundary layer affects the eigenvalues strongly.

To find the eigenfunction and the adjoint eigenfunction corresponding to the eigenvalue $Q_{0}$, we use Eq. (6.10) for the calculation of the correction $Q_{1}$ to the eigenvalue $Q_{0}$, 
and we should in principle calculate the functions $f_{01}(\mathrm{~S}, x, y), f_{02}(\mathrm{~S}, x, y), \bar{\chi}_{01}(\mathrm{~S}, x, y)$ and $\bar{\chi}_{02}(\mathrm{~S}, x, y)$ and solve the systems of equations (6.23), taking into account that their determinants are equal to zero. However, in the process of a direct numerical solution of Eqs. (6.5) and (6.11) over the region of boundary layer (region III) we face the problem of the strong instability of solutions corresponding to the functions $f_{01}$ and $\bar{\chi}_{01}$ with respect to small rapidly increasing disturbances. This instability becomes more pronounced for larger S.

The instability can be illustrated clearly if we pass in Eqs. (6.5) and (6.11) to new variables $\phi(\mathrm{S}, x, y)$ and $\tilde{\phi}(\mathrm{S}, x, y)$ by

$$
f_{0}(\mathrm{~S}, x, y)=C \exp (\phi(\mathrm{S}, x, y)), \quad \bar{\chi}_{0}(\mathrm{~S}, x, y)=\tilde{C} \exp (\tilde{\phi}(\mathrm{S}, x, y))
$$

where

$\phi(\mathrm{S}, x, y)=\int_{0}^{y} B(\mathrm{~S}, x, y) d y, \quad \tilde{\phi}(\mathrm{S}, x, y)=\int_{0}^{y} \tilde{B}(\mathrm{~S}, x, y) d y$.

Substituting (6.29) into Eqs. (6.5) and (6.11) and taking account of (6.30) we obtain the following nonlinear equations for $B(\mathrm{~S}, x, y)$ and $\tilde{B}(\mathrm{~S}, x, y)$ :

$$
\begin{aligned}
& \frac{\partial^{3} B}{\partial y^{3}}+4 B \frac{\partial^{2} B}{\partial y^{2}}+2\left(3 B^{2}-Q_{0}^{2}\right) \frac{\partial B}{\partial y}+3\left(\frac{\partial B}{\partial y}\right)^{2}+\left(B^{2}-Q_{0}^{2}\right)^{2}-\frac{\operatorname{Re}}{2}\left[i ( \mathrm { S } - u _ { \mathrm { d } } ( x , y ) Q _ { 0 } ) \left(\frac{\partial B}{\partial y}\right.\right. \\
& \left.\left.+B^{2}-Q_{0}^{2}\right)+v_{\mathrm{d}}(x, y)\left(\frac{\partial^{2} B}{\partial y^{2}}+3 B \frac{\partial B}{\partial y}+B\left(B^{2}-Q_{0}^{2}\right)\right)+i Q_{0} \Omega_{\mathrm{d} y}(x, y)+\Omega_{\mathrm{d} x}(x, y) B\right]=0 \\
& \frac{\partial^{3} \tilde{B}}{\partial y^{3}}+4 \tilde{B} \frac{\partial^{2} \tilde{B}}{\partial y^{2}}+2\left(3 \tilde{B}^{2}-Q_{0}^{2}\right) \frac{\partial \tilde{B}}{\partial y}+3\left(\frac{\partial B}{\partial y}\right)^{2}+\left(\tilde{B}^{2}-Q_{0}^{2}\right)^{2} \\
& -\frac{\operatorname{Re}}{2}\left[i\left(\mathrm{~S}-u_{\mathrm{d}}(x, y) Q_{0}\right)\left(\frac{\partial \tilde{B}}{\partial y}+\tilde{B}^{2}-Q_{0}^{2}\right)-i Q_{0}\left(2 u_{\mathrm{d} y}(x, y) \tilde{B}+u_{\mathrm{d} y y}(x, y)\right)\right. \\
& -v_{\mathrm{d}}(x, y)\left(\frac{\partial^{2} \tilde{B}}{\partial y^{2}}+3 \tilde{B} \frac{\partial \tilde{B}}{\partial y}+\tilde{B}\left(\tilde{B}^{2}-Q_{0}^{2}\right)\right)-3 v_{\mathrm{d} y}(x, y)\left(\frac{\partial \tilde{B}}{\partial y}+\tilde{B}^{2}-\frac{Q_{0}^{2}}{3}\right) \\
& \left.-3 v_{\mathrm{d} y y}(x, y) \tilde{B}-v_{\mathrm{d} y y y}(x, y)+i Q_{0} \Omega_{\mathrm{d} y}(x, y)-\Omega_{\mathrm{d} x}(x, y) \tilde{B}-\Omega_{\mathrm{d} x y}(x, y)\right]=0 .
\end{aligned}
$$

It is convenient to solve Eqs. (6.31) and (6.32) forward from $y=y_{1}(x)$ to $y=1$ and backward from $y=y_{2}(x)$ to $y=1$, and then to sew the solutions found for $y=1$. In the 
first case we should find four partial solutions of these equations with initial conditions

$$
B_{1,2}\left(\mathrm{~S}, x, y_{1}\right)=\tilde{B}_{1,2}\left(\mathrm{~S}, x, y_{1}\right)= \pm Q_{0}, \quad B_{3,4}\left(\mathrm{~S}, x, y_{1}\right)=\tilde{B}_{3,4}\left(\mathrm{~S}, x, y_{1}\right)= \pm B_{12}
$$

It is evident that the functions $f_{01}(\mathrm{~S}, x, y), f_{02}(\mathrm{~S}, x, y), \bar{\chi}_{01}(\mathrm{~S}, x, y)$ and $\bar{\chi}_{02}(\mathrm{~S}, x, y)$, for $y_{1} \leq|y| \leq 1$, are equal to

$$
\begin{aligned}
& f_{01}(\mathrm{~S}, x, y)=\frac{A_{1}}{2}\left(\exp \left(\phi_{1}(\mathrm{~S}, x, y)\right)-\exp \left(\phi_{2}(\mathrm{~S}, x, y)\right)\right) \\
& f_{02}(\mathrm{~S}, x, y)=\frac{A_{2}}{2}\left(\exp \left(\phi_{3}(\mathrm{~S}, x, y)\right)-\exp \left(\phi_{4}(\mathrm{~S}, x, y)\right)\right), \\
& \bar{\chi}_{01}(\mathrm{~S}, x, y)=\frac{\tilde{A}_{1}}{2}\left(\exp \left(\tilde{\phi}_{1}(\mathrm{~S}, x, y)\right)-\exp \left(\tilde{\phi}_{2}(\mathrm{~S}, x, y)\right)\right), \\
& \bar{\chi}_{02}(\mathrm{~S}, x, y)=\frac{\tilde{A}_{2}}{2}\left(\exp \left(\tilde{\phi}_{3}(\mathrm{~S}, x, y)\right)-\exp \left(\tilde{\phi}_{4}(\mathrm{~S}, x, y)\right)\right),
\end{aligned}
$$

where

$$
\begin{gathered}
\frac{\partial \phi_{1,2}(\mathrm{~S}, x, y)}{\partial y}=B_{1,2}(\mathrm{~S}, x, y), \quad \frac{\partial \tilde{\phi}_{1,2}(\mathrm{~S}, x, y)}{\partial y}=\tilde{B}_{1,2}(\mathrm{~S}, x, y) \\
\frac{\partial \phi_{3,4}(\mathrm{~S}, x, y)}{\partial y}=B_{3,4}(\mathrm{~S}, x, y), \quad \frac{\partial \tilde{\phi}_{3,4}(\mathrm{~S}, x, y)}{\partial y}=\tilde{B}_{3,4}(\mathrm{~S}, x, y), \quad\left(y_{1} \leq|y| \leq 1\right) .
\end{gathered}
$$

In the second case we should find two partial solutions of Eqs. (6.31) and (6.32) with initial conditions

$B_{1}\left(\mathrm{~S}, x, y_{2}\right)=B_{21}, \quad B_{2}\left(\mathrm{~S}, x, y_{2}\right)=B_{22}, \quad \tilde{B}_{1}\left(\mathrm{~S}, x, y_{2}\right)=\tilde{B}_{21}, \quad \tilde{B}_{2}\left(\mathrm{~S}, x, y_{2}\right)=\tilde{B}_{22}$,

where $B_{21}, B_{22}, \tilde{B}_{21}$ and $\tilde{B}_{22}$ are defined by (6.22). The functions $f_{01}(\mathrm{~S}, x, y), f_{02}(\mathrm{~S}, x, y)$, $\bar{\chi}_{01}(\mathrm{~S}, x, y)$ and $\bar{\chi}_{02}(\mathrm{~S}, x, y)$, for $1 \leq|y| \leq y_{2}$, are equal to

$$
\begin{array}{ll}
f_{01}(\mathrm{~S}, x, y)=C_{1} \exp \left(\Phi_{1}(\mathrm{~S}, x, y)\right), & f_{02}(\mathrm{~S}, x, y)=C_{2} \exp \left(\Phi_{2}(\mathrm{~S}, x, y)\right), \\
\bar{\chi}_{01}(\mathrm{~S}, x, y)=\tilde{C}_{1} \exp \left(\tilde{\Phi}_{1}(\mathrm{~S}, x, y)\right), & \bar{\chi}_{02}(\mathrm{~S}, x, y)=\tilde{C}_{2} \exp \left(\tilde{\Phi}_{2}(\mathrm{~S}, x, y)\right),
\end{array}
$$

where

$$
\frac{\partial \Phi_{1,2}(\mathrm{~S}, x, y)}{\partial y}=B_{1,2}(\mathrm{~S}, x, y), \quad \frac{\partial \tilde{\Phi}_{1,2}(\mathrm{~S}, x, y)}{\partial y}=\tilde{B}_{1,2}(\mathrm{~S}, x, y), \quad\left(1 \leq|y| \leq y_{2}\right) .
$$


Numerical solution of Eqs. (6.31) and (6.32), both forward and backward, has shown that in the first case all partial solutions for $B$ tend to the third partial solution with initial condition $\left.B\right|_{y=y_{1}}=B_{12}$, whereas in the second case the first partial solution tends to the second one with initial condition $\left.B\right|_{y=y_{2}}=B_{22}$ (see, for example, Fig. 26, where all numerical partial solutions of Eqs. (6.31) and (6.32) with initial conditions (6.33) and (6.36) are shown for $S=22$ ). This means that all partial solutions, except the third one in the first case and the second one in the second case, are unstable. That is why we have taken the two partial solutions of the equations for $B$ and $\tilde{B}$, following from the Euler equations, as the first and second approximate partial solutions of Eqs. (6.31) and (6.32), and, for $y_{1} \leq y \leq 1$, ignored the fourth partial solution with a large negative real part. The former is valid because $\left|Q_{0}\right| \sim\left|B_{21}\right| \ll \sqrt{\mathrm{Re}}$, and the latter is valid because, for $y_{1} \leq y \leq 1, \exp \left(\int B_{3}(\mathrm{~S}, x, y) d y\right) \gg \exp \left(\int B_{4}(\mathrm{~S}, x, y) d y\right)$. The equations for $B$ and $\tilde{B}$ following from the Euler equations are

$$
\begin{aligned}
& i\left(\mathrm{~S}-u_{\mathrm{d}}(x, y) Q_{0}\right)\left(\frac{\partial B}{\partial y}+B^{2}-Q_{0}^{2}\right)+i Q_{0} \Omega_{\mathrm{d} y}(x, y)+\Omega_{\mathrm{d} x}(x, y) B=0 \\
& i\left(\mathrm{~S}-u_{\mathrm{d}}(x, y) Q_{0}\right)\left(\frac{\partial \tilde{B}}{\partial y}+\tilde{B}^{2}-Q_{0}^{2}\right)-i Q_{0}\left(2 u_{\mathrm{d} y}(x, y) \tilde{B}+u_{\mathrm{d} y y}(x, y)\right) \\
& +i Q_{0} \Omega_{\mathrm{d} y}(x, y)-\Omega_{\mathrm{d} x}(x, y) \tilde{B}-\Omega_{\mathrm{d} x y}(x, y)=0 .
\end{aligned}
$$

An example of the partial solutions found in this way is given in Fig. 27 for $S=22$. Comparing Figs. 27 with 26 we see that, as distinct from the case shown in Fig. 26, all the solutions found are stable.

To find the eigenfunctions and adjoint eigenfunctions we have to use expressions (6.34), (6.37) and, respectively, for the adjoint functions, (6.35), (6.38) and the sewing conditions for $y=1$. Thus we find the equations for $A_{1}, A_{2}, C_{1}$ and $C_{2}$ (and, correspondingly, in the case of the adjoint eigenfunctions, for $\tilde{A}_{1}, \tilde{A}_{2}, \tilde{C}_{1}$ and $\left.\tilde{C}_{2}\right)$ : 


$$
\begin{aligned}
& \frac{A_{1}}{2}\left(\exp \left(\phi_{1}(\mathrm{~S}, x, 1)\right)-\exp \left(\phi_{2}(\mathrm{~S}, x, 1)\right)+\frac{A_{2}}{2} \exp \left(\phi_{3}(\mathrm{~S}, x, 1)\right)\right. \\
& =C_{1} \exp \left(\Phi_{1}(\mathrm{~S}, x, 1)\right)+C_{2} \exp \left(\Phi_{2}(\mathrm{~S}, x, 1)\right) \\
& \frac{A_{1}}{2}\left(B_{1}^{(\mathrm{l})}(\mathrm{S}, x, 1) \exp \left(\phi_{1}(\mathrm{~S}, x, 1)\right)-B_{2}^{(\mathrm{l})}(\mathrm{S}, x, 1) \exp \left(\phi_{2}(\mathrm{~S}, x, 1)\right)\right) \\
& +\frac{A_{2}}{2} B_{3}^{(1)}(\mathrm{S}, x, 1) \exp \left(\phi_{3}(\mathrm{~S}, x, 1)\right) \\
& =C_{1} B_{1}^{(\mathrm{r})}(\mathrm{S}, x, 1) \exp \left(\Phi_{1}(\mathrm{~S}, x, 1)\right)+C_{2} B_{2} 1^{(\mathrm{r})}(\mathrm{S}, x, 1) \exp \left(\Phi_{2}(\mathrm{~S}, x, 1)\right), \\
& \frac{A_{1}}{2}\left(\left(B_{1}^{(\mathrm{l})}(\mathrm{S}, x, 1)\right)^{2} \exp \left(\phi_{1}(\mathrm{~S}, x, 1)\right)-\left(B_{2}^{(\mathrm{l})}(\mathrm{S}, x, 1)\right)^{2} \exp \left(\phi_{2}(\mathrm{~S}, x, 1)\right)\right) \\
& +\frac{A_{2}}{2}\left(B_{3}^{(1)}(\mathrm{S}, x, 1)\right)^{2} \exp \left(\phi_{3}(\mathrm{~S}, x, 1)\right) \\
& =C_{1}\left(B_{1}^{(\mathrm{r})}(\mathrm{S}, x, 1)\right)^{2} \exp \left(\Phi_{1}(\mathrm{~S}, x, 1)\right)+C_{2}\left(B_{2}^{(\mathrm{r})}(\mathrm{S}, x, 1)\right)^{2} \exp \left(\Phi_{2}(\mathrm{~S}, x, 1)\right), \\
& \frac{A_{1}}{2}\left(\left(B_{1}^{(\mathrm{l})}(\mathrm{S}, x, 1)\right)^{3} \exp \left(\phi_{1}(\mathrm{~S}, x, 1)\right)-\left(B_{2}^{(\mathrm{l})}(\mathrm{S}, x, 1)\right)^{3} \exp \left(\phi_{2}(\mathrm{~S}, x, 1)\right)\right) \\
& +\frac{A_{2}}{2}\left(B_{3}^{(\mathrm{l})}(\mathrm{S}, x, 1)\right)^{3} \exp \left(\phi_{3}(\mathrm{~S}, x, 1)\right) \\
& =C_{1}\left(B_{1}^{(\mathrm{r})}(\mathrm{S}, x, 1)\right)^{3} \exp \left(\Phi_{1}(\mathrm{~S}, x, 1)\right)+C_{2}\left(B_{2}^{(\mathrm{r})}(\mathrm{S}, x, 1)\right)^{3} \exp \left(\Phi_{2}(\mathrm{~S}, x, 1)\right),
\end{aligned}
$$

$$
\begin{aligned}
& \frac{\tilde{A}_{1}}{2}\left(\exp \left(\tilde{\phi}_{1}(\mathrm{~S}, x, 1)\right)-\exp \left(\tilde{\phi}_{2}(\mathrm{~S}, x, 1)\right)+\frac{\tilde{A}_{2}}{2} \exp \left(\tilde{\phi}_{3}(\mathrm{~S}, x, y)\right)\right. \\
& =\tilde{C}_{1} \exp \left(\tilde{\Phi}_{1}(\mathrm{~S}, x, 1)\right)+\tilde{C}_{2} \exp \left(\tilde{\Phi}_{2}(\mathrm{~S}, x, 1)\right) \\
& \frac{\tilde{A}_{1}}{2}\left(\tilde{B}_{1}^{(1)}(\mathrm{S}, x, 1) \exp \left(\tilde{\phi}_{1}(\mathrm{~S}, x, 1)\right)-\tilde{B}_{2}^{(1)}(\mathrm{S}, x, 1) \exp \left(\tilde{\phi}_{2}(\mathrm{~S}, x, 1)\right)\right) \\
& +\frac{\tilde{A}_{2}}{2} \tilde{B}_{3}^{(1)}(\mathrm{S}, x, 1) \exp \left(\tilde{\phi}_{3}(\mathrm{~S}, x, 1)\right) \\
& =\tilde{C}_{1} \tilde{B}_{1}^{(\mathrm{r})}(\mathrm{S}, x, 1) \exp \left(\tilde{\Phi}_{1}(\mathrm{~S}, x, 1)\right)+\tilde{C}_{2} \tilde{B}_{2} 1^{(\mathrm{r})}(\mathrm{S}, x, 1) \exp \left(\tilde{\Phi}_{2}(\mathrm{~S}, x, 1)\right), \\
& \frac{\tilde{A}_{1}}{2}\left(\left(\tilde{B}_{1}^{(1)}(\mathrm{S}, x, 1)\right)^{2} \exp \left(\tilde{\phi}_{1}(\mathrm{~S}, x, 1)\right)-\left(\tilde{B}_{2}^{(1)}(\mathrm{S}, x, 1)\right)^{2} \exp \left(\tilde{\phi}_{2}(\mathrm{~S}, x, 1)\right)\right) \\
& +\frac{\tilde{A}_{2}}{2}\left(\tilde{B}_{3}^{(1)}(\mathrm{S}, x, 1)\right)^{2} \exp \left(\tilde{\phi}_{3}(\mathrm{~S}, x, 1)\right) \\
& =\tilde{C}_{1}\left(\tilde{B}_{1}^{(\mathrm{r})}(\mathrm{S}, x, 1)\right)^{2} \exp \left(\tilde{\Phi}_{1}(\mathrm{~S}, x, 1)\right)+\tilde{C}_{2}\left(\tilde{B}_{2}^{(\mathrm{r})}(\mathrm{S}, x, 1)\right)^{2} \exp \left(\tilde{\Phi}_{2}(\mathrm{~S}, x, 1)\right), \\
& \frac{\tilde{A}_{1}}{2}\left(\left(\tilde{B}_{1}^{(1)}(\mathrm{S}, x, 1)\right)^{3} \exp \left(\tilde{\phi}_{1}(\mathrm{~S}, x, 1)\right)-\left(\tilde{B}_{2}^{(\mathrm{l})}(\mathrm{S}, x, 1)\right)^{3} \exp \left(\tilde{\phi}_{2}(\mathrm{~S}, x, 1)\right)\right) \\
& +\frac{\tilde{A}_{2}}{2}\left(\tilde{B}_{3}^{(1)}(\mathrm{S}, x, 1)\right)^{3} \exp \left(\tilde{\phi}_{3}(\mathrm{~S}, x, 1)\right) \\
& =\tilde{C}_{1}\left(\tilde{B}_{1}^{(\mathrm{r})}(\mathrm{S}, x, 1)\right)^{3} \exp \left(\tilde{\Phi}_{1}(\mathrm{~S}, x, 1)\right)+\tilde{C}_{2}\left(\tilde{B}_{2}^{(\mathrm{r})}(\mathrm{S}, x, 1)\right)^{3} \exp \left(\tilde{\Phi}_{2}(\mathrm{~S}, x, 1)\right) .
\end{aligned}
$$


Because the determinants of the systems of equations (6.41) and (6.42) are equal to zero, these equations allow us to find $a=A_{2} / A_{1}, c_{1}=C_{1} / A_{1}$ and $c_{2}=C_{2} / A_{1}$ (and, correspondingly, $\tilde{a}=\tilde{A}_{2} / \tilde{A}_{1}, \tilde{c}_{1}=\tilde{C}_{1} / \tilde{A}_{1}$ and $\left.\tilde{c}_{2}=\tilde{C}_{2} / \tilde{A}_{1}\right)$. Examples of the eigenfunctions and adjoint eigenfunctions constructed in this way are illustrated in Figs. 28, 29 for $x=0$, (a) $\mathrm{S}=10$ and $(b) \mathrm{S}=18$. We see that the range of the sharp change of the eigenfunctions and adjoint eigenfunctions becomes narrower with increasing $\mathrm{S}$.

It is important that the first partial solution of Eq. (6.39) for $y_{1} \leq y \leq 1, B_{1}^{(1)}(\mathrm{S}, x, y)$, transforms uninterruptedly into the first partial solution of the same equation for $1 \leq$ $y \leq y_{2}, B_{1}^{(\mathrm{r})}(\mathrm{S}, x, y)$ (see Fig. 30). Thus, at a point ${ }^{9} y=y^{*}(\mathrm{~S}, x)>1$ the real and imaginary parts of $B_{1}(\mathrm{~S}, x, y)$ change sign. This change of sign provides an explanation of the formation of vortices within the boundary layer: on different sides of the pivot point the stochastic constituents of the longitudinal velocity are oppositely directed.

For $\mathrm{S}<3$, the form of functions $f_{02}(\mathrm{~S}, x, y)$ and $\bar{\chi}_{02}(\mathrm{~S}, x, y)$ means that over the region of the boundary layer (region III) the eigenfunctions $f_{0}(\mathrm{~S}, x, y)$ and adjoint eigenfunctions $\bar{\chi}_{0}(\mathrm{~S}, x, y)$ depend strongly on the Reynolds number and differ markedly from those found from the Euler equations. This is illustrated in Fig. 31, where these functions calculated by the way indicated above and from the Euler equations are compared for $S=1$. For $S>8$ the eigenfunctions and adjoint eigenfunctions are practically independent of the Reynolds number, and hence may be calculated from the Euler equations.

With a knowledge of the eigenfunctions and adjoint eigenfunctions, we can use Eq. (6.10) to calculate the corrections $Q_{1}$ to the eigenvalues of $Q_{0}$. The values of $\Gamma=\Gamma_{0}+\Gamma_{1} / \lambda$ and wave phase velocity $v_{\mathrm{ph}}=\mathrm{S} /\left(K_{0}+K_{1} / \lambda\right)$ as functions of St are shown for different $x$ in Fig. $32 \mathrm{a}$ and $b$. Comparing Fig. 32 a and 24 a we see that for $x<1$ the corrections to the eigenvalues of $\Gamma_{0}$ are not small. This is caused by a rather large value of the derivative of $\Gamma_{0}$ with respect to $x$ for small $x$. The derivatives of $\Gamma_{0}$ and $v_{\mathrm{ph} 0}$ for different values of $x$ are shown in Fig. 33. As $x$ increases the derivatives of $\Gamma_{0}$ and $v_{\mathrm{ph} 0}$, and corrections to the eigenvalues of $Q_{0}$ become progressively smaller.

It can be seen that, as the distance from the nozzle increases, the gain factor decreases

$\overline{9}$ This point is called the pivot point. 
for large St and increases slightly for small St. For any given $x$ the gain factor has a maximum at $\mathrm{St}=\mathrm{St}_{\mathrm{m}}$, where the greater $x$ is the smaller $\mathrm{St}_{\mathrm{m}}$ becomes. It is easily shown that the shift of the gain factor maximum to the low-frequency region is caused by the jet's divergence. Obviously, this shift of the gain factor maximum results in a shift of the turbulent pulsation power spectrum towards the low-frequency region as the distance from the nozzle increases (see below). It is interesting that, from $x \approx 1$, the dependence of $\mathrm{St}_{\mathrm{m}}$ on $x$ is of an exponential character.

Another important conclusion that can be drawn from Fig. 32 lies in the fact that the phase velocity of the hydrodynamic waves depends strongly on the Strouhal number, i.e. these waves are rather significantly dispersive.

Note also that the resonant character of the dependences of the gain factor on the Strouhal number that we have found indicates that each jet cross-section can be considered as an oscillator whose natural frequency decreases with increasing distance from the nozzle. This fact justifies consideration of a jet as a chain of coupled resonant amplifiers, which in turn allows us to understand the analogy between noise-induced pendulum oscillations and the turbulent processes in a jet.

Neglecting the correction to the eigenfunction $f_{0}(\mathrm{~S}, x, y)$, we can write the generative solutions $\psi_{0}(t, x, y), u_{0}(t, x, y)$ and $\Omega_{0}(t, x, y)$ as

$$
\begin{aligned}
& \psi_{0}(t, x, y) \approx \frac{1}{2 \pi} \int_{-\infty}^{\infty} f_{0}(\mathrm{~S}, x, y) \exp \left(i \mathrm{~S} t-i \int_{0}^{x} Q(\mathrm{~S}, x) d x\right) d \mathrm{~S} \\
& u_{0}(t, x, y) \approx \frac{1}{2 \pi} \int_{-\infty}^{\infty} \frac{\partial f_{0}(\mathrm{~S}, x, y)}{\partial y} \exp \left(i \mathrm{~S} t-i \int_{0}^{x} Q(\mathrm{~S}, x) d x\right) d \mathrm{~S} \\
& \Omega_{0}(t, x, y) \approx \frac{1}{2 \pi} \int_{-\infty}^{\infty}\left[\frac{\partial^{2} f_{0}(\mathrm{~S}, x, y)}{\partial y^{2}}-\left(Q^{2}(\mathrm{~S}, x)+i \frac{\partial Q_{0}(\mathrm{~S}, x)}{\partial x}\right) f_{0}(\mathrm{~S}, x, y)\right. \\
& \left.+i Q(\mathrm{~S}, x) \frac{\partial f_{0}(\mathrm{~S}, x, y)}{\partial x}\right] \exp \left(i \mathrm{~S} t-i \int_{0}^{x} Q(\mathrm{~S}, x) d x\right) d \mathrm{~S} .
\end{aligned}
$$

It follows from (6.43) that the vorticity is moderately small outside the boundary layer.

Knowing $Q(\mathrm{~S}, x)$ and the expressions for $u_{0}(t, x, y)$ and $f_{0}(\mathrm{~S}, x, y)$ we can calculate the evolution of the velocity power spectra in the linear approximation. For the sake of sim- 
plicity, we do so only for the region I. We can expand a random disturbance $\xi(t, y)$ of the longitudinal component of velocity at the nozzle exit into a series in the eigenfunctions of our boundary value problem. Over region I we can approximate $\cosh (Q(\mathrm{~S}, 0) y)$ with eigenvalues of $Q$ as the eigenfunctions for $x=0$. Hence, we can set

$$
\xi(t, y)=\xi_{1}(t) \cosh (Q(\mathrm{~S}, 0) y)+\ldots
$$

The spectral density $\left\langle A_{1}(\mathrm{~S}) \bar{A}_{1}(\mathrm{~S})\right\rangle=\left\langle A^{2}(\mathrm{~S})\right\rangle$, where $A(\mathrm{~S})=\left|A_{1}\right|(\mathrm{S})$, is determined by the spectral density of $\xi_{1}(t)$ which is denoted by us as $\kappa(\mathrm{S}, 0)$. Because over region I

$$
u(\mathrm{~S}, 0, y) \approx A_{1}(\mathrm{~S}) Q(\mathrm{~S}, x) \cosh (Q(\mathrm{~S}, 0) y)
$$

we find

$$
\left\langle A^{2}(\mathrm{~S})\right\rangle=\frac{\kappa(\mathrm{S}, 0)}{K^{2}(\mathrm{~S}, 0)+\Gamma^{2}(\mathrm{~S}, 0)} .
$$

There is almost no experimental information about $\kappa(\mathrm{S}, 0)$, but there is one work [133] giving power spectra of the longitudinal and transverse constituents of velocity pulsations over the range of the Strouhal numbers St 0-8 for a circular jet at different initial turbulence levels. It can be seen from these data that the form of the spectra depends only slightly on the initial turbulence level, that the spectra of the longitudinal and transverse constituents of velocity pulsations are nearly identical, and that the spectral density decreases with increasing St. Since the dependence of the spectral densities presented in [133] on $y$ is close to $f(\mathrm{~S})|\cosh (Q(\mathrm{~S}, 0) y)|^{2}$, where $f(\mathrm{~S})$ is a certain function of S, we can set $\kappa(\mathrm{S}, 0) \approx f(\mathrm{~S})$. So, in accordance with data presented in [133], we approximate $\kappa(\mathrm{S}, 0)$ by the formula:

$$
\kappa(\mathrm{S}, 0)=\frac{\kappa_{0}}{1+b_{1} \mathrm{~S}+b_{2} \mathrm{~S}^{2}+b_{3} \mathrm{~S}^{3}}
$$

where $\kappa_{0}$ characterizes the level of the disturbances at the nozzle exit, $b_{1}=0.152, b_{2}=$ -0.005 and $b_{3}=0.000002$. The plot of $\kappa(\mathrm{S}, 0) / \kappa_{0}$ described by $(6.47)$ is shown in Fig. 34 . Comparison with experimental results for power spectra of velocity pulsations and for the mean longitudinal velocity shows that $\kappa_{0}$ should be taken as very small. Hereinafter we 
will set $\kappa_{0}=8 \times 10^{-28}$. It should be noted that, owing to the resonant character of the gain factor, the results are scarcely affected by the shape of the dependence of $\kappa(\mathrm{S}, 0)$.

It follows from (6.43), (6.45) and (6.46) that the spectral density of the longitudinal velocity pulsations in the linear approximation is

$$
\kappa_{\mathrm{l}}(\mathrm{S}, x, y)=\kappa(\mathrm{S}, 0) \frac{K^{2}(\mathrm{~S}, x)+\Gamma^{2}(\mathrm{~S}, x)}{K^{2}(\mathrm{~S}, 0)+\Gamma^{2}(\mathrm{~S}, 0)}|\cosh (Q(\mathrm{~S}, x) y)|^{2} \exp \left(\int_{0}^{x} 2 \Gamma\left(\mathrm{S}, x^{\prime}\right) d x^{\prime}\right) .
$$

The evolutions of $\kappa_{1}(\mathrm{~S}, x, y)$ for $y=0$ and $|y|=0.7$ are shown in Fig. 35 a and $b$. We see that in the two cases considered the power spectra differ markedly, especially for small $x$. In particular, the difference shows up as a faster (for $|y|=0.7$ ) decrease of the Strouhal number corresponding to the spectrum maximum $\left(\mathrm{St}_{\mathrm{m}}\right)$ with increasing distance from the nozzle. This is more easily seen in Fig. 36, where this decrease is given for both cases considered. As is evident from the figures, the experimental dependences can be wellapproximated by the curves $\mathrm{St}_{\mathrm{m}} \approx 3.2 x^{-0.36}$ (for $y=0$ ) and $\mathrm{St}_{\mathrm{m}} \approx 6.5 x^{-0.68}$ (for $y=0.7$ ). Unfortunately, experimental data (see Figs. 5 and 6) are available only for the jet axis (corresponding to $y=0$ ) and a line offset by the radius from the axis (corresponding to $y=1$ ). In the first case our dependence is close to the experimental one, and in the second case it lies between the experimental ones.

We emphasize that our results are obtained from the linear theory without taking account of nonlinear phenomena such as the pairing of vortices. These results reinforce our idea that the experimentally observed shift of the power spectrum is explained mainly by the divergence of the jet, not by the pairing of vortices.

It should be noted that, as the distance from the nozzle increases, the width of the power spectra decreases significantly. This means that the correlation time increases, i.e. the coherence level increases too. This is a cause of the formation of coherent structures.

The mean-root-square value of the turbulent velocity pulsations, which is what is usually 
is measured experimentally, is equal to

$$
\epsilon_{u l}(x, y)=\sqrt{\frac{1}{\pi} \int_{0}^{\infty} \kappa_{\mathrm{l}}(\mathrm{S}, x, y) d \mathrm{~S} .}
$$

The plots of $\epsilon_{u 1}(x, 0)$ and $\epsilon_{u l}(x, 0.7)$ versus $y$ are presented in Fig. 37. It is seen from Fig. 37 a that the dependence of $\epsilon_{u l}(x, 0)$ on $x$ closely resembles the dependence of an order parameter on temperature for a slightly noisy second-order phase transition. This also reinforces our hypothesis that the onset of turbulence is a nonequilibrium noiseinduced phase transition of the second order, similar to that for a pendulum with a randomly vibrated suspension axis.

It is interesting that for $y=0.7$ the root-mean-square value of turbulent velocity pulsations first decreases and then increases with increasing $x$. This can be explained by the competition between the amplification of the pulsations and the swift decrease of the spectrum width. The condition for validity of all results obtained in this paper is that $\epsilon_{u l}(x, y) \ll 1$. We see that along the jet axis the results are valid for almost the whole initial part $(x<8)$, whereas for $y=0.7$ they are valid only for $x<5$. The change of $\epsilon(x, y)$ as $x$ increases is correlated with the change of the mean velocity (see below), but these changes are not fully identical.

Let us trace the changes of the group wave velocity at $\mathrm{St}=\mathrm{St}_{\mathrm{m}}$, as well as of the wave lengths in the longitudinal and transverse directions $\left(\lambda_{\mathrm{lon}}\left(\mathrm{St}_{\mathrm{m}}, x\right)=2 \pi / K\left(\mathrm{St}_{\mathrm{m}}, x\right)\right.$ and $\lambda_{\text {tr }}\left(\mathrm{St}_{\mathrm{m}}, x\right)=2 \pi / \Gamma\left(\mathrm{St}_{\mathrm{m}}, x\right)$, respectively), with increasing $x$. The results are presented in Fig. 38. It is seen that along the jet axis, as the distance from the nozzle increases, the group velocity $v_{\mathrm{gr}}\left(\mathrm{St}_{\mathrm{m}}(x)\right)$ first decreases and then increases, whereas for $y=0.7$ it decreases monotonically. The longitudinal wave lengths increase considerably in both cases, providing evidence for an increase in the scale of the turbulence in the longitudinal direction. Along the jet axis the transverse scale of turbulence increases too, whereas for $y=0.7$ it changes nonmonotonically. The increase of the scale of turbulence in the longitudinal direction agrees with the experimental data and reveals itself in the pairing of vortices. 


\subsection{The first approximation}

Putting

$$
\frac{\partial A_{1}}{\partial x} \sim \frac{\partial A_{1}}{\partial y} \sim \epsilon^{2}
$$

substituting (6.1) into Eqs. (5.36), (5.37) and equating the terms of order $\epsilon$ we obtain the following equations:

$r_{1}-\Delta s_{1}=0, \quad q_{1}=\frac{\partial s_{1}}{\partial y}$

$\frac{\partial r_{1}}{\partial t}+u_{\mathrm{d}}(x, y) \frac{\partial r_{1}}{\partial x}+v_{\mathrm{d}}(x, y) \frac{\partial r_{1}}{\partial y}-\Omega_{\mathrm{d} y}(x, y) \frac{\partial s_{1}}{\partial x}+\Omega_{\mathrm{d} x}(x, y) \frac{\partial s_{1}}{\partial y}-\frac{2}{\operatorname{Re}} \Delta r_{1}=R(t, x, y)$

where

$$
R(t, x, y)=\frac{\partial \psi_{0}}{\partial x} \frac{\partial \Omega_{0}}{\partial y}-\frac{\partial \Omega_{0}}{\partial x} \frac{\partial \psi_{0}}{\partial y}
$$

and $\psi_{0}$ and $\Omega_{0}$ are defined by the expressions (6.43).

We suppose that only waves for which the sign of $\mathrm{S}$ is opposite can interact, an assumption that is justified for dispersive waves. It should be noted that the interaction of waves with the same sign of $S$ results in the generation of second harmonic, whereas the interaction of waves for which $\mathrm{S}$ is opposite in sign results in the appearance of a constant level. Because the difference

$$
\frac{\partial \psi_{0}^{(\mathrm{S})}}{\partial x} \frac{\partial \Omega_{0}^{(\mathrm{S})}}{\partial y}-\frac{\partial \Omega_{0}^{(\mathrm{S})}}{\partial x} \frac{\partial \psi_{0}^{(\mathrm{S})}}{\partial y}
$$

is small, the second harmonic is also small. It is therefore sufficient to consider the interaction only of waves for which $\mathrm{S}$ is opposite in sign. In so doing, it should be borne in mind that $A_{1}(-\mathrm{S})=\bar{A}_{1}(\mathrm{~S})$ and $Q(-\mathrm{S})=-\bar{Q}(\mathrm{~S})$. Thus, we can set $A_{1}(\mathrm{~S}) A_{1}\left(\mathrm{~S}^{\prime}\right)=$ $2 \pi A^{2}(\mathrm{~S}) \delta\left(\mathrm{S}^{\prime}+\mathrm{S}\right)$, where $A(\mathrm{~S})=\left|A_{1}\right|(\mathrm{S})$. In this case $R(t, x, y)$ is independent of $t$, and we can represent it as

$$
R(x, y)=\frac{1}{2 \pi} \int_{-\infty}^{\infty} R_{s}(\mathrm{~S}, x, y) d \mathrm{~S}
$$

A solution of Eqs. (6.50) can be also presented in the form 


$$
\begin{aligned}
& r_{1}(x, y)=\frac{1}{2 \pi} \int_{-\infty}^{\infty} r_{1 s}(\mathrm{~S}, x, y) d \mathrm{~S}, \quad s_{1}(x, y)=\frac{1}{2 \pi} \int_{-\infty}^{\infty} s_{1 s}(\mathrm{~S}, x, y) d \mathrm{~S}, \\
& q_{1}(x, y)=\frac{1}{2 \pi} \int_{-\infty}^{\infty} q_{1 s}(\mathrm{~S}, x, y) d \mathrm{~S},
\end{aligned}
$$

Equations (6.50) can be solved analytically only over regions I, II.

It follows from (6.43) and our numerical calculations that over region I

$$
\begin{aligned}
& \psi_{0}(\mathrm{~S}, x, y) \approx A_{1} \sinh (Q(\mathrm{~S}, x) y) \exp \left(i \mathrm{~S} t-i \int_{0}^{x} Q(\mathrm{~S}, x) d x\right) \\
& \Omega_{0}(\mathrm{~S}, x, y) \approx-i A_{1}[\sinh (Q(\mathrm{~S}, x) y)+2 Q(\mathrm{~S}, x) y \cosh (Q(\mathrm{~S}, x) y)] \frac{\partial Q(\mathrm{~S}, x)}{\partial x} \\
& \times \exp \left(i \mathrm{~S} t-i \int_{0}^{x} Q(\mathrm{~S}, x) d x\right),
\end{aligned}
$$

and in region II

$$
\begin{aligned}
& \psi_{0}(\mathrm{~S}, x, y) \approx A_{1}\left[c_{1}(\mathrm{~S}, x) \exp \left(B_{21}(\mathrm{~S}, x)\left(y-y_{2}(x)\right)\right)+c_{2}(\mathrm{~S}, x)\right. \\
& \times \exp \left(B_{22}(\mathrm{~S}, x)\left(y-y_{2}(x)\right)\right] \exp \left(i \mathrm{~S} t-i \int_{0}^{x} Q(\mathrm{~S}, x) d x\right) \\
& \Omega_{0}(\mathrm{~S}, x, y) \approx A_{1}\left[\frac{Q(\mathrm{~S}, x) \Omega_{\mathrm{d} x}(x, \infty)}{i \mathrm{~S}-Q(\mathrm{~S}, x) v_{\mathrm{d} x}(x, \infty)} c_{1}(\mathrm{~S}, x) \exp \left(B_{21}(\mathrm{~S}, x)\left(y-y_{2}(x)\right)\right)+\right. \\
& B_{22}^{2}(\mathrm{~S}, x) c_{2}(\mathrm{~S}, x) \exp \left(B_{22}(\mathrm{~S}, x)\left(y-y_{2}(x)\right)\right] \exp \left(i \mathrm{~S} t-i \int_{0}^{x} Q(\mathrm{~S}, x) d x\right)
\end{aligned}
$$

where $c_{1}(\mathrm{~S}, x)$ and $c_{2}(\mathrm{~S}, x)$ are found from Eqs. (6.41), and $B_{21}(\mathrm{~S}, x)$ and $B_{22}(\mathrm{~S}, x)$ are determined by (6.21), (6.22). For $y-y_{2}(x) \gg 1 /\left|B_{22}(\mathrm{~S}, x)\right|$ the expressions (6.56) may be reduced to 


$$
\begin{aligned}
& \psi_{0}(\mathrm{~S}, x, y) \approx A_{1} c_{1}(\mathrm{~S}, x) \exp \left(B_{21}(\mathrm{~S}, x)\left(y-y_{2}(x)\right)\right) \exp \left(i \mathrm{~S} t-i \int_{0}^{x} Q(\mathrm{~S}, x) d x\right), \\
& \Omega_{0}(\mathrm{~S}, x, y) \approx A_{1} \frac{Q(\mathrm{~S}, x) \Omega_{\mathrm{d} x}(x, \infty)}{i \mathrm{~S}-Q(\mathrm{~S}, x) v_{\mathrm{d} x}(x, \infty)} c_{1}(\mathrm{~S}, x) \exp \left(B_{21}(\mathrm{~S}, x)\left(y-y_{2}(x)\right)\right) \\
& \exp \left(i \mathrm{~S} t-i \int_{0}^{x} Q(\mathrm{~S}, x) d x\right) .
\end{aligned}
$$

\subsubsection{Region I}

It follows from (6.55) that

$$
\begin{aligned}
& R_{s}(\mathrm{~S}, x, y)=-2\left(K^{2}(\mathrm{~S}, x)+\Gamma^{2}(\mathrm{~S}, x)\right)\left[2 \frac{\partial K(\mathrm{~S}, x)}{\partial x} \sinh (2 K(\mathrm{~S}, x) y)+\frac{\partial \Gamma(\mathrm{S}, x)}{\partial x}\right. \\
& \left.\times \sin (2 \Gamma(\mathrm{S}, x) y)+2\left(K(\mathrm{~S}, x) \frac{\partial K(\mathrm{~S}, x)}{\partial x}-\Gamma(\mathrm{S}, x) \frac{\partial \Gamma(\mathrm{S}, x)}{\partial x}\right) y \cosh (2 K(\mathrm{~S}, x) y)\right] \\
& \times A^{2}(\mathrm{~S}) \exp \left(2 \int_{0}^{x} \Gamma(\mathrm{S}, x) d x\right) .
\end{aligned}
$$

Eqs. (6.50) for $r_{1 s}, q_{1 s}$ and $s_{1 s}$ become

$$
r_{1 s}-\Delta s_{1 s}=0, \quad q_{1 s}=\frac{\partial s_{1 s}}{\partial y}, \quad \frac{\partial r_{1 s}}{\partial x}-\frac{2}{\operatorname{Re}} \Delta r_{1 s}=R_{s}(\mathrm{~S}, x, y) .
$$

Substituting (6.59) into Eqs. (6.60), and ignoring the term proportional to 1/Re, we obtain the following approximate expressions for $r_{1 s}(\mathrm{~S}, x, y), s_{1 s}(\mathrm{~S}, x, y)$ and $q_{1 s}(\mathrm{~S}, x, y)$ :

$$
\begin{aligned}
& r_{1 s}(\mathrm{~S}, x, y)=\tilde{r}_{1 s}(\mathrm{~S}, x, y) A^{2}(\mathrm{~S}) \exp \left(2 \int_{0}^{x} \Gamma(\mathrm{S}, x) d x\right) . \\
& s_{1 s}(\mathrm{~S}, x, y)=\tilde{s}_{1 s}(\mathrm{~S}, x, y) A^{2}(\mathrm{~S}) \exp \left(2 \int_{0}^{x} \Gamma(\mathrm{S}, x) d x\right), \\
& q_{1 s}(\mathrm{~S}, x, y)=\tilde{q}_{1 s}(\mathrm{~S}, x, y) A^{2}(\mathrm{~S}) \exp \left(2 \int_{0}^{x} \Gamma(\mathrm{S}, x) d x\right),
\end{aligned}
$$

where

$$
\tilde{r}_{1 s}(\mathrm{~S}, x, y) \approx-\frac{K^{2}(\mathrm{~S}, x)+\Gamma^{2}(\mathrm{~S}, x)}{\Gamma(\mathrm{S}, x)}\left\{2 \frac{\partial K(\mathrm{~S}, x)}{\partial x} \sinh (2 K(\mathrm{~S}, x) y)+\frac{\partial \Gamma(\mathrm{S}, x)}{\partial x}\right.
$$




$$
\begin{aligned}
& \left.\times \sin (2 \Gamma(\mathrm{S}, x) y)+2\left(K(\mathrm{~S}, x) \frac{\partial K(\mathrm{~S}, x)}{\partial x}-\Gamma(\mathrm{S}, x) \frac{\partial \Gamma(\mathrm{S}, x)}{\partial x}\right) y \cosh (2 K(\mathrm{~S}, x) y)\right\} \\
& \tilde{s}_{1 s}(\mathrm{~S}, x, y) \approx-\frac{1}{2 \Gamma(\mathrm{S}, x)}\left[\frac{\Gamma(\mathrm{S}, x)}{K^{2}(\mathrm{~S}, x)+\Gamma^{2}(\mathrm{~S}, x)}\left(\Gamma \frac{\partial K(\mathrm{~S}, x)}{\partial x}+K(\mathrm{~S}, x) \frac{\partial \Gamma(\mathrm{S}, x)}{\partial x}\right)\right. \\
& \times \sinh (2 K(\mathrm{~S}, x) y)+\left(K(\mathrm{~S}, x) \frac{\partial K(\mathrm{~S}, x)}{\partial x}-\Gamma(\mathrm{S}, x) \frac{\partial \Gamma(\mathrm{S}, x)}{\partial x}\right) y \cosh (2 K(\mathrm{~S}, x) y) \\
& \left.-\frac{K^{2}(\mathrm{~S}, x)+\Gamma^{2}(\mathrm{~S}, x)}{2 \Gamma(\mathrm{S}, x)} \frac{\partial \Gamma(\mathrm{S}, x)}{\partial x} y \cos (2 \Gamma(\mathrm{S}, x) y)\right]
\end{aligned}
$$

$$
\begin{aligned}
& \tilde{q}_{1 s}(\mathrm{~S}, x, y) \approx-\frac{1}{2 \Gamma(\mathrm{S}, x)}\left\{\frac { 1 } { K ^ { 2 } ( \mathrm { S } , x ) + \Gamma ^ { 2 } ( \mathrm { S } , x ) } \left(\left(3 \Gamma^{2}(\mathrm{~S}, x)+K^{2}(\mathrm{~S}, x)\right) K(\mathrm{~S}, x) \frac{\partial K(\mathrm{~S}, x)}{\partial x}\right.\right. \\
& \left.+\left(K^{2}(\mathrm{~S}, x)-\Gamma^{2}(\mathrm{~S}, x)\right) \Gamma(\mathrm{S}, x) \frac{\partial \Gamma(\mathrm{S}, x)}{\partial x}\right) \cosh (2 K(\mathrm{~S}, x) y)+2 K(\mathrm{~S}, x) y\left(K(\mathrm{~S}, x) \frac{\partial K(\mathrm{~S}, x)}{\partial x}\right. \\
& \left.-\Gamma(\mathrm{S}, x) \frac{\partial \Gamma(\mathrm{S}, x)}{\partial x}\right) \sinh (2 K(\mathrm{~S}, x) y)-\frac{K^{2}(\mathrm{~S}, x)+\Gamma^{2}(\mathrm{~S}, x)}{2 \Gamma(\mathrm{S}, x)} \frac{\partial \Gamma(\mathrm{S}, x)}{\partial x} \\
& \times[\cos (2 \Gamma(\mathrm{S}, x) y)-2 \Gamma(\mathrm{S}, x) y \sin (2 \Gamma(\mathrm{S}, x) y)]\} .
\end{aligned}
$$

It follows from (6.64) that along the jet axis, where $\sinh (2 K(\mathrm{~S}, x) y)=\sin (2 \Gamma(\mathrm{S}, x) y)=0$ and $\cosh (2 K(\mathrm{~S}, x) y)=\cos (2 \Gamma(\mathrm{S}, x) y)=1$, we have $r_{1 s}(\mathrm{~S}, x, 0)=s_{1 s}(\mathrm{~S}, x, 0)=0$ and

$$
\begin{aligned}
& \tilde{q}_{1 s}(\mathrm{~S}, x, y) \approx-\frac{1}{2 \Gamma(\mathrm{S}, x)}\left\{\frac { 1 } { K ^ { 2 } ( \mathrm { S } , x ) + \Gamma ^ { 2 } ( \mathrm { S } , x ) } \left(\left(3 \Gamma^{2}(\mathrm{~S}, x)+K^{2}(\mathrm{~S}, x)\right) K(\mathrm{~S}, x) \frac{\partial K(\mathrm{~S}, x)}{\partial x}\right.\right. \\
& \left.\left.+\left(K^{2}(\mathrm{~S}, x)-\Gamma^{2}(\mathrm{~S}, x)\right) \Gamma(\mathrm{S}, x) \frac{\partial \Gamma(\mathrm{S}, x)}{\partial x}\right)-\frac{K^{2}(\mathrm{~S}, x)+\Gamma^{2}(\mathrm{~S}, x)}{2 \Gamma(\mathrm{S}, x)} \frac{\partial \Gamma(\mathrm{S}, x)}{\partial x}\right\} .
\end{aligned}
$$

It should be noted that the functions $\tilde{r}_{1 s}, \tilde{s}_{1 s}$ and $\tilde{q}_{1 s}$ are slow functions of $x$.

We emphasize that the function $\left\langle q_{1}(x, y)\right\rangle$ determines the dependence on $x$ and $y$ of the additional constant correction to the dynamical constituent of the longitudinal velocity, i.e. the change of the mean velocity due to nonlinear effects. This change is caused by turbulent pulsations. It is intuitively obvious (and confirmed by experiment) that the correction found over region I must be negative, i.e. turbulent pulsations must decrease the mean flow velocity in region I.

Averaging (6.62), substituting (6.46) into $\left\langle q_{1 s}(\mathrm{~S}, x, y)\right\rangle$ and integrating over $\mathbf{S}$ we can find $\left\langle q_{1}(x, y)\right\rangle$ for different values of $x$ and $y \leq y_{1}(x)$. Fig. 39 shows some examples of the 
dependences of $\langle u(x, y)\rangle=u_{\mathrm{d}}(x, y)+\left\langle q_{1}(x, y)\right\rangle$ on $y$ at fixed values of $x$, and on $x$ at fixed values of $y$. It is seen from Fig. 39 a that the mean velocity profile becomes increasingly bell-shaped as $x$ increases. Furthermore (see Fig. 39 b), within the initial part $(x \leq 8)$ the velocity on the jet axis decreases significantly only at its end, and then by no more than $5 \%$. Off-axis, the velocity falls off much faster.

\subsubsection{Region II}

It follows from (6.58) and (6.21) that

$$
\begin{aligned}
& R_{s}(\mathrm{~S}, x, y)=-\frac{4 \Omega_{\mathrm{d} x}(x, \infty)\left|c_{1}(\mathrm{~S}, x)\right|^{2} K(\mathrm{~S}, x) \mathrm{S}}{\left(K^{2}(\mathrm{~S}, x)+\Gamma^{2}(\mathrm{~S}, x)\right)^{2} v_{\mathrm{d}}^{2}(x, \infty)+\mathrm{S}\left(\mathrm{S}-2 \Gamma(\mathrm{S}, x) v_{\mathrm{d}}(x, \infty)\right)} \\
& \times\left(K^{2}(\mathrm{~S}, x)+\Gamma^{2}(\mathrm{~S}, x)-\frac{\Omega_{\mathrm{d} x}(x, \infty)\left[\left(K^{2}(\mathrm{~S}, x)-\Gamma^{2}(\mathrm{~S}, x)\right) v_{\mathrm{d}}(x, \infty)+\Gamma(\mathrm{S}, x) \mathrm{S}\right]}{2\left[K^{2}(\mathrm{~S}, x) v_{\mathrm{d}}^{2}(x, \infty)+\left(\mathrm{S}-\Gamma(\mathrm{S}, x) v_{\mathrm{d}}(x, \infty)\right)^{2}\right]}\right) \\
& \times \exp \left\{2\left[B_{21 \mathrm{r}}(\mathrm{S}, x)\left(y-y_{2}(x)\right)+\int_{0}^{x} \Gamma(\mathrm{S}, x) d x\right]\right\} A^{2}(\mathrm{~S}),
\end{aligned}
$$

where

$$
B_{21 \mathrm{r}}(\mathrm{S}, x)=-K(\mathrm{~S}, x)\left(1-\frac{\Omega_{\mathrm{d} x}(x, \infty) v_{\mathrm{d}}(x, \infty)}{2\left[K^{2}(\mathrm{~S}, x) v_{\mathrm{d}}^{2}(x, \infty)+\left(\mathrm{S}-\Gamma(\mathrm{S}, x) v_{\mathrm{d}}(x, \infty)\right)^{2}\right]}\right) .
$$

Equations (6.50) for $r_{1 s}$ and $s_{1 s}$ become

$$
\begin{aligned}
& r_{1 s}-\Delta s_{1 s}=0 \\
& v_{\mathrm{d}}(x, \infty) \frac{\partial r_{1 s}}{\partial y}+\Omega_{\mathrm{dx}}(x, \infty) \frac{\partial s_{1 s}}{\partial y}-\frac{2}{\operatorname{Re}} \Delta r_{1 s}=R_{s}(x, y),
\end{aligned}
$$

Ignoring the term proportional to $1 / \mathrm{Re}$, we obtain the following approximate expressions for $r_{1 s}(\mathrm{~S}, x, y), s_{1 s}(\mathrm{~S}, x, y)$ and $q_{1 s}(\mathrm{~S}, x, y)$ :

$$
r_{1 s}(\mathrm{~S}, x, y) \approx \frac{2 R_{s}(\mathrm{~S}, x, y)\left(B_{21 \mathrm{r}}^{2}(\mathrm{~S}, x)+\Gamma^{2}(\mathrm{~S}, x)\right)}{B_{21 \mathrm{r}}(\mathrm{S}, x)\left[4\left(B_{21 \mathrm{r}}^{2}(\mathrm{~S}, x)+\Gamma^{2}(\mathrm{~S}, x)\right) v_{\mathrm{d}}(x, \infty)+\Omega_{\mathrm{dx}}(x, \infty)\right]}
$$




$$
\begin{aligned}
& s_{1 s}(\mathrm{~S}, x, y) \approx \frac{R_{s}(\mathrm{~S}, x, y)}{2 B_{21 \mathrm{r}}(\mathrm{S}, x)\left[4\left(B_{21 \mathrm{r}}^{2}(\mathrm{~S}, x)+\Gamma^{2}(\mathrm{~S}, x)\right) v_{\mathrm{d}}(x, \infty)+\Omega_{\mathrm{dx}}(x, \infty)\right]} \\
& q_{1 s}(\mathrm{~S}, x, y) \approx \frac{R_{s}(\mathrm{~S}, x, y)}{4\left(B_{21 \mathrm{r}}^{2}(\mathrm{~S}, x)+\Gamma^{2}(\mathrm{~S}, x)\right) v_{\mathrm{d}}(x, \infty)+\Omega_{\mathrm{dx}}(x, \infty)}
\end{aligned}
$$

Averaging (6.69), substituting (6.46) into $\left\langle q_{1 s}(\mathrm{~S}, x, y)\right\rangle$ and integrating over $\mathbf{S}$ we can find $\left\langle q_{1}(x, y)\right\rangle$ for different values of $x$ and $y \geq y_{2}(x)$. Extrapolating the values of $\left\langle q_{1}(x, y)\right\rangle$ found here and for region I into the region of boundary layer we can estimate the mean longitudinal velocity profiles width for different values of $x$, taking account of stochastic constituents. An example of such profile for $x=8$ (the end of the initial part) is given in Fig. 40 a. For comparison, the corresponding profile of the dynamical constituent of the velocity is shown in the same figure. We see that these profiles differ substantially. It should be emphasized that the velocity profile that we have found by taking account of the stochastic constituents coincides in form with experimentally measured profiles.

Unfortunately, we cannot calculate exactly the full velocity profiles, or the change of velocity, for all $y$ because we are restricted to regions I and II and take no account of strong nonlinear effects. However, we can calculate the width of the internal and external parts of the boundary layer. If we take for the internal boundary the plane where the mean velocity is equal to $0.95 U_{0}$, and for the external boundary the plane where the mean velocity is equal to $0.05 U_{0}$, then these boundaries are as shown in Fig. 40 b. Our results demonstrate that the boundaries of the mixing layer are very far from being the straight lines adduced by many researchers. Up to a certain value of $x$, these boundaries nearly coincide with the boundaries of the mixing layer for the dynamical constituents. Only for larger $x$ do they strongly move apart.

\subsection{The second approximation}

To derive the equation for the amplitude $A_{1}$ in the second approximation, we set in the expansion (6.1) 


$$
\begin{aligned}
& r_{2}(t, x, y)=\frac{1}{2 \pi} \int_{-\infty}^{\infty} C(\mathrm{~S}) \sinh (Q(\mathrm{~S}, x) y) \exp \left(i \mathrm{~S} t-i \int_{0}^{x} Q(\mathrm{~S}, x) d x\right) d \mathrm{~S} \\
& s_{2}(t, x, y)=\frac{1}{2 \pi} \int_{-\infty}^{\infty} D(\mathrm{~S}) \sinh (Q(\mathrm{~S}, x) y) \exp \left(i \mathrm{~S} t-i \int_{0}^{x} Q(\mathrm{~S}, x) d x\right) d \mathrm{~S}
\end{aligned}
$$

where $C(\mathrm{~S}) \sim \epsilon^{2}$ and $D(\mathrm{~S}) \sim \epsilon^{2}$ are unknown amplitudes.

Like the first approximation, the second approximation can be found analytically only over regions I and II. For simplicity, we restrict our consideration to region I.

Equating the terms of order $\epsilon^{2}$ in Eqs. (5.36), (5.37) for region I, neglecting the derivatives of $Q(\mathrm{~S}, x)$ with respect to $x$ and taking account of (6.70), we obtain the following system of approximate equations for $C$ and $D$ with determinant equal to zero:

$$
\begin{aligned}
& C=-2 Q(\mathrm{~S}, x)\left(i \frac{\partial A_{1}}{\partial x}-\frac{\partial A_{1}}{\partial y} \operatorname{coth}(Q(\mathrm{~S}, x) y)\right), \\
& i(\mathrm{~S}-Q(\mathrm{~S}, x)) C=-Q(\mathrm{~S}, x)\left(\frac{\partial r_{1}(x, y)}{\partial x} \operatorname{coth}(Q(\mathrm{~S}, x) y)+i \frac{\partial r_{1}(x, y)}{\partial y}\right) A_{1},
\end{aligned}
$$

where $r_{1}(x, y)$ is defined by $(6.53),(6.61)$.

From the condition of compatibility of Eqs. (6.71), we find a truncated equation for the amplitude $A_{1}$ over region I. It can be written as

$$
\tanh (Q(\mathrm{~S}, x) y) \frac{\partial A_{1}}{\partial x}+i \frac{\partial A_{1}}{\partial y}=\Phi(\mathrm{S}, x, y) A_{1}
$$

where

$$
\begin{aligned}
& \Phi(\mathrm{S}, x, y)=\frac{1}{2 \pi} \int_{-\infty}^{\infty} F(\mathrm{~S}, \mathrm{~s}, x, y) A_{0}^{2}(\mathrm{~s}) \exp \left(2 \int_{0}^{x} \Gamma\left(\mathrm{s}, x^{\prime}\right) d x^{\prime}\right) d \mathbf{s} \\
& \left.F(\mathrm{~S}, \mathrm{~s}, x, y)=\frac{1}{2(Q(\mathrm{~S}, x)-\mathrm{S})}\left[2 \Gamma(\mathrm{s}, x) \tilde{r}_{1 s}(\mathrm{~s}, x, y)\right)+i \tanh (Q(\mathrm{~S}, x) y) \frac{\partial \tilde{r}_{1 s}(\mathrm{~s}, x, y)}{\partial y}\right] \\
& \text { and } A_{0}(\mathrm{~S})=\left|A_{1}(\mathrm{~S}, 0,0)\right| .
\end{aligned}
$$


Equation (6.72) can be conveniently rewritten in terms of

$$
z(\mathrm{~S}, x, y)=\ln \left(\frac{A_{1}(\mathrm{~S}, x, y)}{A_{1}(\mathrm{~S}, 0,0)}\right)
$$

as

$$
\tanh (Q(\mathrm{~S}, x) y) \frac{\partial z}{\partial x}+i \frac{\partial z}{\partial y}=\Phi(\mathrm{S}, x, y)
$$

A solution of Eq. (6.76) can be represented as

$$
z(\mathrm{~S}, x, y)=\frac{1}{2 \pi} \int_{-\infty}^{\infty} w(\mathrm{~S}, \mathrm{~s}, x, y) \exp \left(2 \int_{0}^{x} \Gamma\left(\mathrm{s}, x^{\prime}\right) d x^{\prime}\right) d \mathbf{s}
$$

Substituting (6.77) into Eq. (6.76), taking into account that $F(\mathbf{S}, \mathbf{s}, x, y)$ and $w(\mathbf{S}, \mathbf{s}, x, y)$ are slow functions of $x$, and neglecting the derivative of $w$ with respect to $x$ we obtain the following equation for $w(\mathbf{S}, \mathbf{s}, x, y)$ :

$$
\frac{\partial w}{\partial y}-2 i \Gamma(\mathbf{s}, x) \tanh (Q(\mathrm{~S}, x) y) w=-i F(\mathbf{S}, \mathbf{s}, x, y) A_{0}^{2}(\mathbf{s})
$$

The solution of Eq. (6.78) with initial condition $w(\mathrm{~S}, \mathbf{s}, x, 0)=0$ is

$$
w(\mathrm{~S}, \mathbf{s}, x, y)=-i A_{0}^{2}(\mathbf{s}) \int_{0}^{y} F\left(\mathrm{~S}, \mathbf{s}, x, y^{\prime}\right)\left(\frac{\cosh (Q(\mathrm{~S}, x) y)}{\cosh \left(Q(\mathrm{~S}, x) y^{\prime}\right)}\right)^{-2 i \Gamma(\mathbf{s}, x) / Q(\mathrm{~S}, x)} d y^{\prime}
$$

After substituting (6.79) into (6.77) and integrating over s, we find $z(\mathrm{~S}, x, y)$.

Knowledge of $z(\mathrm{~S}, x, y)$, in view of $(6.75)$, allows us to find $\left\langle A^{2}(\mathrm{~S}, x, y)\right\rangle$ and the mean value of the phase shift. Taking account of (6.75) and using the assumption that $|z(\mathrm{~S}, x, y)| \ll 1$ we find

$$
\begin{aligned}
& \left\langle A^{2}(\mathrm{~S}, x, y)\right\rangle \approx\left\langle A_{0}^{2}(\mathrm{~S})\right\rangle(1+2 \operatorname{Re}[\langle z(\mathrm{~S}, x, y)\rangle]), \\
& \langle\varphi(\mathrm{S}, x, y)-\varphi(\mathrm{S}, 0,0)\rangle \approx \operatorname{Im}[\langle z(\mathrm{~S}, x, y)\rangle]
\end{aligned}
$$

where 


$$
\begin{aligned}
& \langle z(\mathrm{~S}, x, y)\rangle=-\frac{i}{2 \pi} \int_{-\infty}^{\infty} \frac{\kappa(\mathrm{s}, 0)}{K^{2}(\mathrm{~s}, 0)+\Gamma^{2}(\mathrm{~s}, 0)} \exp \left(2 \int_{0}^{x} \Gamma\left(\mathrm{s}, x^{\prime}\right) d x^{\prime}\right) \\
& \times \int_{0}^{y} F\left(\mathrm{~S}, \mathrm{~s}, x, y^{\prime}\right)\left(\frac{\cosh (Q(\mathrm{~S}, x) y)}{\cosh \left(Q(\mathrm{~S}, x) y^{\prime}\right)}\right)^{-2 i \Gamma(\mathrm{s}, x) / Q(\mathrm{~S}, x)} d y^{\prime} d \mathbf{s},
\end{aligned}
$$

$\kappa(\mathrm{S}, 0)$ is defined by $(6.47)$.

The spectral constituent of the longitudinal velocity pulsations is

$$
\begin{aligned}
u(\mathrm{~S}, x, y)=\left(A_{1}(\mathrm{~S}, x, y) Q(\mathrm{~S}, x) \cosh (Q(\mathrm{~S}, x) y)+\right. & \left.\frac{\partial A_{1}(\mathrm{~S}, x, y)}{\partial y} \sinh (Q(\mathrm{~S}, x) y)\right)(6.8 \\
& \times \exp \left(i \mathrm{St}-i \int_{0}^{x} Q(\mathrm{~S}, x) d x\right) .
\end{aligned}
$$

Because of the smallness of $z(\mathrm{~S}, x, y)$ we have $A_{1}(\mathrm{~S}, x, y) \approx A_{0}(\mathrm{~S})(1+z(\mathrm{~S}, x, y))$. It follows from here that the spectral density of the longitudinal velocity pulsations, with account taken of nonlinearity, can be represented as

$$
\kappa(\mathrm{S}, x, y)=\kappa_{\mathrm{l}}(\mathrm{S}, x, y)\left(1+2\langle z(\mathrm{~S}, x, y)\rangle+\frac{2 \tanh (Q(\mathrm{~S}, x) y)}{Q(\mathrm{~S}, x)} \frac{\partial\langle z(\mathrm{~S}, x, y)\rangle}{\partial y}\right)
$$

where $\kappa_{1}(\mathrm{~S}, x, y)$ is determined by $(6.48)$.

It follows from (6.81) that for $y=0$, in the approximation under consideration, the relative nonlinear correction

$$
\Delta \kappa(\mathrm{S}, x, y)=2\langle z(\mathrm{~S}, x, y)\rangle+\frac{2 \tanh (Q(\mathrm{~S}, x) y)}{Q(\mathrm{~S}, x)} \frac{\partial\langle z(\mathrm{~S}, x, y)\rangle}{\partial y}
$$

to the spectral density $\kappa_{\mathrm{l}}(\mathrm{S}, x, y)$ is absent. For $y \neq 0$, however, this correction is essential, and it increases with increasing $y$ and $x$. Examples of the dependences of $\Delta \kappa$ on the Strouhal number St for $y=0.7$ and a number values of $x$ and for $x=8$ and three values of $y$ are given in Fig. $41 \mathrm{a}$ and $b$, respectively. It is seen that for $x \leq 5$ and $y=0.7$ the nonlinear correction changes nonmonotonically with increasing $x$, even changing its sign. Only for $x>5$ the changes become monotone, and nearly for all values of St the correction is negative. The latter means that for these St the nonlinearity causes the saturation of turbulent pulsations. We note that the saturation occurs only from a certain value of 
the Strouhal number. For smaller Strouhal numbers the nonlinear amplification occurs in place of the saturation.

Two examples of the velocity pulsations spectral density (for $x=6$, and $y=0.5$ and 0.7 ) with taking into account of the nonlinearity are illustrated in Fig. 42. For comparison the corresponding spectral densities calculated in the linear approximation are shown in the same figure. It is seen that the nonlinearity results in the significant decrease of the spectral density maximal value, and as a consequence in the decrease of the turbulent pulsations variance. It is seen from Fig. 42 that, for $x=6$, the spectral density for $y=0.5$ is more than for $y=0.7$, even in linear approximation; whereas for small $x$ it increases monotonically with increasing $y$.

We note that $\partial\langle\varphi(\mathrm{S}, x, y)\rangle \equiv \Delta K(\mathrm{~S}, x, y)$ gives a nonlinear correction to the wave number $K(\mathrm{~S}, x)$. It is important to note that this correction depends on the transverse coordinate $y$. It follows from (6.80) and (6.81) that it is

$$
\begin{aligned}
& \Delta K(\mathrm{~S}, x, y) \approx-\int_{-\infty}^{\infty} \frac{\kappa(\mathrm{s}, 0)}{K^{2}(\mathrm{~s}, 0)+\Gamma^{2}(\mathrm{~s}, 0)} \Gamma(\mathrm{s}, x) \exp \left(2 \int_{0}^{x} \Gamma\left(\mathrm{s}, x^{\prime}\right) d x^{\prime}\right) \\
& \times \operatorname{Re}\left[\int_{0}^{y} F\left(\mathrm{~S}, \mathrm{~s}, x, y^{\prime}\right)\left(\frac{\cosh (Q(\mathrm{~S}, x) y)}{\cosh \left(Q(\mathrm{~S}, x) y^{\prime}\right)}\right)^{-2 i \Gamma(\mathrm{s}, x) / Q(\mathrm{~S}, x)} d y^{\prime}\right] d \mathrm{~s} .
\end{aligned}
$$

The value of $\Delta K(\mathrm{~S}, x, y)$ determines a nonlinear correction $\Delta \lambda_{\text {lon }}$ to the longitudinal wave length $\lambda_{\text {lon }}(\mathrm{S}, x)=2 \pi / K(\mathrm{~S}, x)$ :

$$
\Delta \lambda_{\text {lon }}=-\frac{2 \pi \Delta K(\mathrm{~S}, x, y)}{K^{2}(\mathrm{~S}, x)}
$$

Because nearly for all St the values of $\Delta K$ are negative, we can conclude that the nonlinearity causes the faster increase of turbulence scales with increasing $x$, in comparison with the results of linear consideration. 


\section{Conclusions}

The theoretical approach proposed above has enabled us to account for many experimental results, and to demonstrate that a number of widely-accepted interpretations are in fact erroneous. It has led us to a somewhat different and, we believe, more realistic perspective. In particular -

(1) Our studies show that the shift of velocity pulsation power spectra to the lowfrequency domain is caused mainly by the jet divergence, not pairing of vortices, so that it can therefore be calculated within the linear approximation.

(2) The observed phenomenon of vortices pairing can be accounted for in terms of the increase in the longitudinal and transverse turbulent scales, which is caused by jet divergence and not by resonance relations.

(3) The transformation of the mean velocity profile can be found without the use of the concept of turbulent viscosity.

(4) The influence of nonlinearity close to the jet symmetry plane $(y=0)$ is very small within the initial part of the jet, but increases significantly as we approach the boundary layer.

(5) The intensity of random disturbances at the nozzle exit necessary for the onset of turbulence may be very small. Our quasi-linear theory is valid only for such small intensities. For larger disturbance intensities, the development of turbulence is from the very outset an essentially nonlinear process.

(6) The change of the velocity pulsation variance with distance from the nozzle closely resembles changing order parameter with increasing temperature for a second order phase transition. That is why we guess that the onset of turbulence can be considered as a specific noise-induced phase transition similar to that for a pendulum with a randomly vibrated suspension axis. 


\section{Acknowledgements}

It is a pleasure to acknowledge useful discussions with G. Kolmakov and helpful advices related to computation of A. Sil'chenko and I. Kaufman. The research was supported in part by the Engineering and Physical Sciences Research Council (UK), and by the Royal Society of London to whom PSL is indebted for a visiting research fellowship at Lancaster during which much of the review was completed.

\section{References}

[1] G. Hagen, Über die Bewegung des Wassers in engen zylidrischen Röhren, Pogg. Ann. 46 (1839) 423-442.

[2] O. Reynolds, An experimental investigation of the circumstances which determine whether the motion of water shall be direct os sinouos, and the law of resistance in parallel channels, Phil. Trans. Roy. Soc., London, 174 (1883) 935-982.

[3] G. Compte-Bellot, Écoulement Turbulent Entre deux Parois Paralléles (Éditeé par le service de documentation scientifique et technique de l'armement, Paris, 1965).

[4] A.E. Ginevsky, Ye.V. Vlasov and R.K. Karavosov, Acoustic Control of Turbulence Jets, (Fizmatlit, Moscow, 2001) (in Russian); English Translation: Springer, Heidelberg, 2004 (to be published).

[5] C.C. Lin, The Theory of Hydrodynamic Stability (Cambridge Univ. Press, Cambridge, $1955)$.

[6] S. Chandrasekhar, Hydrodynamic and Hydromagnetic Stability (Clarendon Press, Oxford, 1961).

[7] R. Betchov and W.O. Criminale, Stability of Parallel Flows (Acad. Press, New York, 1967).

[8] M.A. Goldshtik and V.N. Sctern, Hydrodynamic Stability and Turbulence (Nauka, Novosibirsk, 1977) (in Russian).

[9] P.D. Drazin and W.H. Reid, Hydrodynamic Stability, (Cambridge Univ. Press, Cambridge, 1981). 
[10] Yu.S. Kachanov, V.V. Kozlov and V.Ya. Levchenko, The Onset of Turbulence in a Boundary Layer, (Novosibirsk, Nauka, 1982) (in Russian).

[11] L.D. Landau and E.M. Lifshitz, Hydrodynamics (Nauka, Moscow, 1986) (in Russian).

[12] A.S. Monin and A.M. Yaglom, Statistical Gydromechanics, vol. 1, 2 (Gidrometeoizdat, Sankt-Petersburg, 1992) (in Russian).

[13] L. Prandtl, Führer durch die Strömungslehre (F. Vieweg, Braunschweig, 1949).

[14] H. Schlichting, Grenzschicht-Theorie (Verlag G. Braun, Karlsruhe, 1965).

[15] M.A. Goldshtik, V.N. Shtern and N.I. Yavorsky, Viscous Flows with Paradoxical Properties (Nauka, Novosibirsk, 1989) (in Russian).

[16] Yu.L. Klimontovich, Turbulent Motion and the Structures of Chaos (Kluwer Academic Publ., Dordrecht, 1991).

[17] P. Holmes, J.L. Lumley and G. Berkooz, Turbulence, Coherent Structures and Symmetry, (Cambridge Univ. Press, Cambridge, 1996).

[18] R.J. Roache, Computational Fluid Dynamics, (Hermosa Publishers Albuquerque, 1976).

[19] P. Bradshaw, T. Cebeci and J.H. Whitelaw, Engineering Calculation Methods for Turbulent Flow (Acad. Press, New York, 1981).

[20] D.C. Wilcox, Turbulence Modelling for CFD (DCW Industries Inc., La Canada, California, 1998).

[21] S.M. Belotserkovsky and A.S. Ginevsky, Simulation of Turbulent Jets and Wakes by Discrete Vortex Technique (Nauka, Moscow, 1995) (in Russian).

[22] A.N. Kolmogorov, Local structure of turbulence in incompressible fluid for very large Reynolds numbers, DAN SSSR 30 (1941) 299-303 (in Russian).

[23] A.N. Kolmogorov, The energy dispersion in the case of locally isotropic turbulence, DAN SSSR 32 (1941) 19-21 (in Russian).

[24] A.M. Obukhov, On the energy distribution in a power spectrum of a turbulent flow, Izv. AN SSSR, ser. Geogr. i Geofiz., 5 (1941) 453-466 (in Russian).

[25] U. Frisch, Turbulence: The Legacy of A.N. Kolmogorov, (Cambridge Univ. Press, Cambridge, 1995). 
[26] Ye.A. Novikov, Random force method in turbulence theory, ZHETF 44 (1963) 2159-2168 (in Russian).

[27] D. Segel, V.S. L'vov and I. Procaccia, Extended self-similarity in turbulent systems: an analytically solvable example, Phys. Rev. Letters 76 (1996) 1828-1831.

[28] V.S. L'vov and I. Procaccia, Cornerstones of a theory of anomalous scaling in turbulence, Physica Scripta T67 (1996) 131-135.

[29] V.S. L'vov and I. Procaccia, The universal scaling exponents of anisotropy in turbulence and their measurement, Physics of Fluids 8 (1996) 2565-2567.

[30] V.S. L'vov and I. Procaccia. Towards a Nonperturbative Theory of Hydrodynamic Turbulence: Fusion Rules, Exact Bridge Relations and Anomalous Scaling Functions, Phys. Rev. E., 54 (1996), 6268-6284.

[31] V.S. L'vov, E. Podivilov and I. Procaccia. Temporal multiscaling in hydrodynamic turbulence, Phys. Rev. E, 55 (1997) 7030-7035.

[32] A.L. Fairhall, B. Dhruva, V.S. Lvov, I. Procaccia and K.S. Sreenivassan, Fusion rules in Navier-Stokes turbulence: first experimental tests , Phys. Rev. Letters 79 (1997) 3174-3177.

[33] V.S. L'vov, and I. Procaccia, Computing the Scaling Exponents in Fluid Turbulence from First Principles: the Formal Setup, Physica A, 257 (1998) 165-196.

[34] V.I. Belinicher, V.S. L'vov, and I. Procaccia. A new approach to computing the scaling exponents in fluid turbulence from first principles, Physica A, 254 (1998) 215-230 (1998).

[35] V.I. Belinicher, V.S. L'vov, A.Pomyalov and I. Procaccia. Computing the Scaling Exponents in Fluid Turbulence from First Principles: Demonstration of Multiscaling, J. of Stat. Phys., 93 (1998) 797-832.

[36] I. Arad, L. Biferale, I. Mazzitelli and I. Procaccia, Disentangling scaling properties in anisotropic and inhomogeneous turbulence, Phys. Rev. Lett. 82 (1999) 5040-5043.

[37] G. Amati, R. Benzi and S. Succi, Extended self-similarity in boundary layer turbulence, Phys. Rev. E 55 (1997) 6985-6988.

[38] V.S. L'vov and I. Procaccia, Exact resummation in the theory of hydrodynamic turbulence: on line resummed diagrammatic perturbation approach, in: F. David, P. Ginsparg and J. Zinn-Justin, eds., Les Houches session LXII, 1994, Fluctuating Geometries in Statistical Mechanics and Field Theory (Elsevier, New York, 1995). 
[39] V.S. L'vov and I. Procaccia, Anomalous Scaling in Turbulence: a Field Theoretic Approach, in Nonlinear Dynamics, Chaotic and Complex Systems, ed. by E. Infeld, R. Zelazny and A Galkovski, (Cambridge Univ. Press, Cambridge, 1996).

[40] I. Arad, V. S. L'vov and I. Procaccia, Correlation functions in isotropic and anisotropic turbulence: the role of the symmetry group, Phys. Rev. E 59 (1999) 6753-6765.

[41] Chih-Ming Ho and N.S. Nosseir, Large coherent structures in an impinging jet, in: Turbulent Shear Flows 2 (2nd Int. Symp., London, 1979), eds. L.J.S. Bradbury et al. (Springer, Berlin, 1980), pp. 297-304.

[42] Chih-Ming Ho and N.S. Nosseir, Dynamics of an impinging jet 1. The feedback phenomenon, J. Fluid Mech. 105 (1981) 119-142.

[43] H. Curasava, K. Yamanoue and T. Obata, Self-excited oscillation in an axisymmetric jet with a coaxial circular pipe, Mem. Nagano Techn. Coll. No 18 (1987) 21-30.

[44] P.S. Landa, Nonlinear Oscillations and Waves in Dynamical Systems (Kluwer Academic Publ., Dordrecht, 1996).

[45] P.S. Landa, Nonlinear Oscillations and Waves (Nauka, Moscow, 1997) (in Russian).

[46] A.S. Ginevsky and P.S. Landa, Excitation of hydrodynamic and acoustic waves in subsonic jet and separated flows, Izv. vuzov, Prikladnaya Nelineynaya Dinamika 3 (1995) No 2, 4259 (in Russian).

[47] P.S. Landa, Turbulence in nonclosed fluid flows as a noise-induced phase transition, Europhys. Lett. 36 (1996) 401-406.

[48] P.S. Landa, Universality of oscillation theory laws. Types and role of mathematical models, Discrete Dynamics in Nature and Society 1 (1997) 99-110.

[49] P.S. Landa, Onset of turbulence in open liquid flows as a nonequilibrium noise-induced second-order phase transition, Techn. Physics 43 (1998) 27-34.

[50] P.S. Landa, A.S. Ginevsky, Ye.V. Vlasov, and A.A. Zaikin, Turbulence and coherent structures in subsonic submerged jets. Control of the Turbulence, Int. J. Bifurcations and Chaos 9 (1999) 397-414.

[51] Ye.V. Vlasov, A.S. Ginevsky, P.S. Landa and D.L. Samodurov, On analogy between the responce on periodical excitation of a turbulent jet and the pendulum with randomly vibrated suspension axis, J. Eng. Phys. and Thermophys. 75 (2002) No 4, 90-93. 
[52] A.K.M.F. Hussain and C.A. Thompson, Controlled symmetric perturbation of the plain jet: an experimental study in the initial region, J. Fluid Mech. 100 (1980) 397-431.

[53] H.E. Fielder, Coherent structures, Adv. Turbul. Proc. 1st Eur. Turbul. Conf., Lyon, 1-4 July 1987, pp. 320-336.

[54] H.E. Fielder and H.H. Fernholz, On management and control of turbulent shear flows, Progr. Aerospace Sci. 27 (1990) 305-387.

[55] Ye.V. Vlasov, A.S. Ginevsky and V.G. Pimshtein, Control of coherent structures and aeroacoustic characteristics of subsonic and supersonic turbulent jets, Proc DGLR/IAA 14th Aeroacoustics Conf. (1992), Aachen, pp. 672-678.

[56] A.S. Ginevsky, Control of coherent structures and aero-acoustic characteristics of subsonic and supersonic turbulent jets, In: IUTAM Symp. "The Active Control of Vibration", Proc., Bath, 1994, 203-207.

[57] A.S. Ginevsky, Acoustic Methods of Turbulent Mixing Control in Jet Flows, In: The Third Int. Congress on Air- and Structure-Borne Sound and Vibration. Montreal, pp. 1191-1198.

[58] P.S. Landa, A.A. Zaikin, M.G. Rosenblum, and J. Kurths, Control of noise-induced oscillations of a pendulum with a randomly vibrated suspension axis, Phys. Rev. E 56 (1997) 1465-1470.

[59] P.S. Landa, Using Krylov-Bogolyubov asymptotic method for the calculation of the onset of turbulence in subsonic submerged jets. 4th Euromech Nonlinear Oscillations Conference (ENOC-2002), 19-23 August 2002, Moscow, p. 98.

[60] F.K. Browand and J. Laufer, The role of large scale structures in the initial development of circular jets, in: Turbulence in Liquids (Proc. IV Biennial Symp. Turbulence Liquids, Sept. 1975) (Princeton, New York, 1977), 333-344.

[61] J. Laufer and P. Monkevitz, On turbulent jet flows: a new perspective, AIAA Pap. No 962 (1980).

[62] Chih-Ming Ho, Local and global dynamics of free shear layers, in: Numerical and Physical Aspects of Aerodynamic Flows, (Springer-Verlag, New York, 1982), 521-533.

[63] Chih-Ming Ho and P.Huerre, Perturbed free shear layers, Ann. Rev. Fluid Mech. 16 (1984) $365-424$. 
[64] A.K.M.F. Hussain, Coherent structures - reality and myth, Phys. Fluids 26 (1983) 28162859.

[65] Ye.V. Vlasov and A.S. Ginevsky, Coherent structures in turbulent jets and wakes, in: Itogi Nauki i Tekhniki, Mekhanika Zhidkosti i Gasa (VINITI AN SSSR 1986), vol. 20, 3-84 (in Russian).

[66] High Reynolds Number Flows Using Liquid and Gaseous Helium. Discussion of Liquid and Gaseous Helium as a Test Fluid, ed. by R.J. Donnelly, (Springer-Verlag, New York, 1991).

[67] S.M. Rytov, To memory of G.S. Gorelik, UFN 62 (1957) 485-496 (in Russian).

[68] L.D. Landau, On the turbulence problem, DAN SSSR 44 (1944) 339-342 (in Russian).

[69] J.T. Stuart, On the nonlinear mechanics of hydrodynamic stability, J. Fluid Mech. 4 (1958) $1-21$.

[70] J.T. Stuart, On the nonlinear mechanics of wave disturbances in stable and unstable parallel flows, P. 1, J. Fluid Mech. 9 (1960) 353-370.

[71] J.T. Stuart, Hydrodynamic stability, Appl. Mech. Rev. 18 (1965) 523-531.

[72] J.T. Stuart, Nonlinear stability theory, Ann. Rev. Fluid Mech 3 (1971) 347-370.

[73] W. Heisenberg, Über Stabilität und Turbulenz von Flüssigkcitsströmen, Ann. Phys. 74 (1924) 577-595.

[74] D. Ruelle and F. Takens, On the nature of turbulence, Comm. Math. Phys. 20 (1971) $167-192$.

[75] D. Ruelle D. Strange attractors as a mathematical explanation of turbulence, Lect. Notes in Phys., Statistical Models and Turbulence 12 (1975) 292-316.

[76] Yu.I. Neimark and P.S. Landa, Stochastic and Chaotic Oscillations, (Kluwer Academic Publishers, Dordrecht, 1992).

[77] A.V. Gaponov-Grekhov, M.I. Rabinovich and I.M. Starobinets, Dynamical model of the spatial development of turbulence, JETP Letters 39 (1984) 688-691.

[78] G.V. Osipov, On turbulence development according to Landau in a discrete model of flow systems, Izv. vuzov, Radiofizika 31 (1988) 624-632 (in Russian). 
[79] K.I. Artamonov, Thermo-hydro-acoustic stability, (Mashinostroenie, Moscow, 1982) (in Russian).

[80] N.V. Nikitin, Direct numerical simulation of three-dimensional turbulent flows in circular pipes, Izv. RAN, MZhG (1994) No 6 14-26.

[81] N.V. Nikitin, Spatial approach to numerical simulation of turbulence in pipes, DAN 343(1995) 767-770.

[82] H. Salwen and C.E. Grosch, The stability of Poiseuille flow in a pipe of circular crosssection, J. Fluid Mech. 54 (1971) 93-112.

[83] Landa P.S., Rabinovitch A. Exhibition of intrinsic properties of certain systems in response to external disturbances. Phys. Rev. E, 2000, vol. 61, No 2, 1829-1838.

[84] L.A. Vulis and V.P. Kashkarov, The Theory of Jets in Viscous Fluid, (Nauka, Moscow, 1965) (in Russian).

[85] A.A. Townsend, The Structure of Turbulent Shear Flow (Cambridge Univ. Press, Cambridge, 1956).

[86] A.S. Ginevsky, The Theory of Turbulent Jets and Wakes, (Mashinovedenie, Moscow, 1969) (in Russian).

[87] G.N. Abramovich, T.A. Girshovich, S.Yu. Krasheninnikov, A.N. Sekundov, and I.P. Smirnova, Theory of Turbulent Jets, (Nauka, Moscow, 1984) (in Russian).

[88] J.W. Oler and V.W. Goldschmidt, A vortex-street model of the flow in the similarity region of a two-dimensional free turbulent jet, J. Fluid Mech. 123 (1982), pp. 523-535.

[89] F.O. Thomas and E.G. Brehov, An investigation of large-scale structure in the similarity region of a two-dimensional turbulent jet, Phys. Fluids 29 (1986), pp. 1788-1795.

[90] F.O. Thomas and V.W. Goldschmidt, Structural characteristics of a developing turbulent planar jet, J. Fluid Mech. 163 (1986), pp. 227-256.

[91] J. Tso and A.K.M.F. Hussain, Organized motions in a fully developed turbulent axisymmetric jet, J. Fluid Mech. 203 (1989) pp. 425-448.

[92] S.V. Gordeyev and F.O. Thomas, Coherent structure in the turbulent planar jet. Part 1. Extraction of proper orthogonal decomposition eigenmodes and their self-similarity, J. Fluid Mech. 414 (2000), pp. 145-194. 
[93] S.V. Gordeyev and F.O. Thomas, Coherent structure in the turbulent planar jet. Part 2. Structural topology via POD eigenmode projection, J. Fluid Mech. 460 (2002), pp. 349380.

[94] J.O. Hinze, Turbulence. An Introduction to its Mechanism and Theory, (McGraw-Hill Book Company Inc., New York, Toronto, London, 1959).

[95] J.C. Laurence, Intensity, scale and spectra of turbulence in mixing region of free subsonic jet, NACA Rep. 1292 (1956).

[96] R.A. Petersen, Influence of wave dispersion on vortex pairing in a jet, J.Fluid Mech. 89 (1978) 469-495.

[97] H.H. Bruun, A time-domain evolution of the large-scale flow structure in a turbulent jet, Proc. Roy. Soc. (London) A367 (1979) No 1729, pp. 193-218.

[98] S.C. Crow and F.H. Champagne, Orderly structure in jet turbulence, J.Fluid Mech. 48 (1971) 547-591.

[99] Y.Y. Chan, Spatial waves in turbulence jets, Phys. Fluids 17 (1974) 46-53, 1667-1670.

[100] P. Plaschko, Axial coherence functions of circular turbulent jets based on inviscidly calculated damped modes, Physics of Fluids 26 (1983) 2368-2372.

[101] A.S. Ginevsky, Ye.V. Vlasov and A.V. Kolesnikov, Aeroacoustic Interactions, (Mashinostroenie, Moscow, 1978) (in Russian).

[102] Ye.V. Vlasov and A.S. Ginevsky, Acoustic modification of the aerodynamic characteristics of a turbulent jet, Fluid Dynamics 2 (1967) No 4, 93-96.

[103] Ye.V. Vlasov and A.S. Ginevsky, Generation and suppression of turbulence in an axisymmetric turbulent jet in the presence of an acoustic influence, Mekhnika Zhidkosti i Gaza No 6 (1973) 37-43 (in Russian).

[104] Ye.V. Vlasov and A.S. Ginevsky, Problem of aeroacoustic interactions, Soviet Physics Acoustics 26 (1980) 1-7.

[105] Ye.V. Vlasov, A.S. Ginevsky and R.K. Karavosov, Influence of the mode composition of acoustic disturbances on jet aerodynamic characteristics, Soviet Phys. Acoustic 32 (1986) 329-330. 
[106] Ye.V. Vlasov, A.S. Ginevsky, R.K. Karavosov, and T.M. Makarenko, Turbulence suppression in subsonic jets by high-frequency acoustic excitation, Fluid Dynamics 34 (1999) 23-28.

[107] Ye.V. Vlasov, A.S. Ginevsky, R.K. Karavosov, and T.M. Makarenko, On change of the sign of high-frequency acoustic action on a turbulent jet with the increase of excitation, J. Eng. Phys. and Thermophys. 1 (2001) 8-9.

[108] Yu.Ya. Borisov and N.M. Ginkina, Excitation of high-velocity jets by acoustic oscillations, Akusticheskiy Zhurnal 21 (1975) 364-371 (in Russian).

[109] K.M.B.Q. Zaman and A.K.M.F. Hussain, Turbulence suppression in free shear flows by controlled excitation, J. Fluid Mech. 103 (1981) 133-159.

[110] M. Nallasamy, Turbulence suppression at high amplitudes of excitation: numerical and experimental study, Bull. Am. Phys. Soc. 28 (1983) 1380.

[111] M. Nallasamy and A.K.M.F. Hussain, Numerical study of the phenomenon of turbulence suppression in a plane shear layer, Turbulent Shear Flows 4 (1984) 169-181.

[112] M. Nallasamy and A.K.M.F. Hussain, Effects of excitation on turbulence levels in a shear layer, Trans. ASME 111 (1989) 102-104.

[113] V. Kibens, Discrete noise spectrum generated by an acousticly excited jet, AIAA Journal 18 (1980) 434-441.

[114] P.S. Landa and A.A. Zaikin, Noise-induced phase transitions in a pendulum with a randomly vibrated suspension axis, Phys. Rev. E 54 (1996) 3535-3544.

[115] P.S. Landa and A.A. Zaikin, Nonequilibrium noise-induced phase transitions in simple systems, ZhETF 111 (1997) 358-378.

[116] P.S. Landa and A.A. Zaikin, Noise-induced phase transitions in nonlinear oscillators, in: AIP Conference Proceedings 465 (Computing Anticipatory Systems, CASYS'98, Liege, Belgium 1998), 419-433.

[117] P.S. Landa, A.A. Zaikin and J. Kurths, On noise-induced transitions in nonlinear oscillators, in: Stochastic Processes in Physics, Chemistry, and Biology, ed. by J.A. Freynd, T. Pöschel, (Springer, Berlin, 2000), 268-279. 
[118] N. Platt, E.A. Spiegel and C. Tresser, On-off intermittency: a mechanism for bursting, Phys. Rev. Lett. 70 (1993) 279-282.

[119] H. Fujisaka and T. Yamada, A new intermittency in coupled dynamical systems, Progr. Theor. Phys. 74 (1985) 918-921.

[120] J.F. Heagy, N. Platt and S.M. Hammel, Characterization of on-off intermittency, Phys. Rev. E 49 (1994) 1140-1150.

[121] P.S. Landa, A.A. Zaikin, M.G. Rosenblum, and J. Kurths, On-off intermittency phenomena in a pendulum with a randomly vibrated suspension axis, Chaos, Solitons and Fractals 9 (1998) 157-169.

[122] U. Frisch and R. Morf, Intermittency in nonlinear dynamics and singularities at complex times, Phys. Rev. A 23 (1981) 2673-2705.

[123] P.S. Landa and P.V.E. McClintock, Changes in the dynamical behavior of nonlinear systems induced by noise, Physics Reports 323 (2000) 1-80.

[124] H.G. Schuster, Deterministic Chaos, (Physik-Verlag, Weinheim, 1984).

[125] G. Raman, E.J. Rice and R.R. Mankbadi, Saturation and a limit of jet mixing enhancement by single frequency plane wave excitation: experiment and theory, in: Collect Papers, P. 2 (AIAA/ASSME/SIAM/APS, 1st Nat. Fluid Dyn. Congr., Cincinnati, Ohio, 1988), pp. 1000-1007.

[126] D.G. Crighton and M. Gaster, Stability of slowly diverging jet flow, J. Fluid Mech. 77 (1976) 397-413.

[127] P. Plaschko, Helical instabilities of slowly diverging jets, J. Fluid Mech. 92 (1979) 209-215.

[128] A. Michalke, Survey on jet instability theory, Progr. Aerosp. Sci. 21 (1984) 159-199.

[129] M.V. Keldysh, On eigenvalues and eigenfunctions of certain classes of non-self-adjoint equations, DAN SSSR 77 (1951) 11-14.

[130] R. Courant and D. Hilbert, Methods Of Mathematical Physics, Vol. 2, (John Wiley, New York-London, 1962).

[131] P.S. Landa, Self-oscillations in Spatially Extended Systems, (Nauka, Moscow, 1983) (in Russian). 
[132] M. Gaster, On the growth of waves in boundary layers: a non-parallel correction, J. Fluid Mech. 424 (2000) 367-377.

[133] G. Raman, K.B.M.Q. Zaman and E.J. Rice, Initial turbulence effect on jet evolution with and without tonal excitation, Phys. Fluids A 1 (1989) 1240-1248. 


\section{Figure legends}

(1) Instantaneous distributions of the longitudinal velocity component $u$ in a steady regime for $A / u_{0}=0.04$ : a along the pipe axis $(r / R=0.02)$ and $b$ near the pipe wall $(r / R=0.93)$. After [81].

(2) View of the turbulent velocity pulsations in a pipe (a) with periodic boundary conditions and (b) with the boundary conditions (2.1), (2.2). After [80].

(3) (a) Schematic diagram of a diverging free jet illustrating the change of its mean velocity profile and widening of the mixing layer. Curves 1 and 2 correspond to the internal and external boundaries of the mixing layer, respectively. (b) Schematic dependence of the relative mean velocity $U / U_{0}$ along the jet axis on the distance $x$ from the nozzle exit section.

(4) Experimental dependence of the relative mean velocity $U / U_{0}$ along the jet axis on the relative distance $x / D$ from the nozzle exit section, for three intensities of the disturbance at the nozzle exit section: $\epsilon_{u}(0)=0.015,0.0930 .209$ (curves marked by open circles, filled circles and stars, respectively). After [50].

(5) Evolution of the spectral density $S p$ (in decibels) of velocity pulsations $u$ with increasing distance from the nozzle exit $x / D$ along the jet axis $(r=0)$, and along a line offset by $R$ from the axis $(r=1)$. At the bottom, the spectral density for $x / D=0.5$, $r=1$ is shown on a larger scale. After [50].

(6) The experimental dependence of the Strouhal number $\mathrm{St}_{m}$ on the relative distance $x / D$ from the jet nozzle exit along the jet axis and within the mixing layer: (a) Petersen's data for the mixing layer [96]; and (b) the data of [50]. In (b) the dependence on distance along the jet axis, and along a line offset by $R$ from the axis, are shown by squares and circles, respectively. The solid lines show the dependences $\mathrm{St}_{m}=C_{1} x^{-1 / 3}$ and $\mathrm{St}_{m}=C_{2} x^{-1}$, where $C_{1} \approx 0.67$ and $C_{2} \approx 1$.

(7) The experimental dependence of $\epsilon_{u}=\sqrt{\overline{u^{2}}} / U_{0}$ on $\epsilon_{u \mathrm{a}}=\sqrt{\overline{\overline{u_{\mathrm{a}}^{2}}}} / U_{0}$ for $x / D$ and values of the Strouhal number marked near the corresponding curves. After [98].

(8) The dependence of $\epsilon_{u}$ on $\mathrm{St}_{\mathrm{a}}$ for $\epsilon_{u \mathrm{a}}=0.02, x / D=4$ constructed from the data given in Fig. 7. In the absence of acoustical disturbance $\epsilon_{u} \approx 0.04$. After [98].

(9) (a) The experimental dependence on $\tilde{x}=(x / D) \mathrm{St}_{\mathrm{a}}$ of the root-mean-square pulsation of hydrodynamical pressure $\tilde{p}=\sqrt{\overline{p^{2}}}$ (in decibels), in the middle of the mixing layer, 
for a fixed amplitude of acoustic disturbance and for different Strouhal numbers. After [99]. (b) The theoretical dependence on the relative distance from the jet nozzle $x / R$ of the gain factor $K$ for axially symmetric pulsations of hydrodynamical pressure in a circular jet, for $r / R=1.05, \mathrm{St}_{\mathrm{a}}=0.5$. After [100].

(10) The experimental dependences of the relative root-mean-square pulsation of the suppression factors $(\mathrm{a}) \epsilon_{u} / \epsilon_{u}^{(0)}$ and (b) $\epsilon_{v} / \epsilon_{v}^{(0)}$ of the longitudinal and radial components of hydrodynamical velocity on the relative amplitude of acoustic pressure $\tilde{p}_{\text {a }}$ measured in decibels, for $\mathrm{St}_{\mathrm{a}}=2.35, x / D=8 ; \epsilon_{u}^{(0)}$ and $\epsilon_{v}^{(0)}$ are relative pulsations of the longitudinal and radial velocity components in the absence of acoustic excitation. After [65].

(11) Experimental dependences: of (top) the relative mean flow velocity along the jet axis $U / U^{(0)}$ and of the relative root-mean-square pulsation of the longitudinal (middle) $\left(\epsilon_{u}\right)$ and radial (bottom) $\left(\epsilon_{v}\right)$ components of hydrodynamic velocity (in \%) on $x / D$ under a longitudinal acoustic forcing at $\mathrm{St}_{\mathrm{a}}=0.25$ (light circles), $\mathrm{St}_{\mathrm{a}}=2.75$ (filled circles). When the acoustic forcing is absent, the corresponding curves are marked by triangles. The amplitude of the oscillatory velocity in the acoustical wave on the jet axis near the nozzle exit constitutes $0.07 \%$ of $U_{0}$. After [65].

(12) The experimental dependences on the Strouhal number $\mathrm{St}_{\theta}=(\theta / D) \mathrm{St}$ of the suppression factor $\epsilon_{u} / \epsilon_{u}^{(0)}$, where $\epsilon_{u}^{(0)}$ is the relative intensity of the longitudinal velocity pulsations in the absence of acoustic forcing, for $x / \theta=200$. The plots are constructed for four values of the amplitude of the oscillatory velocity in the acoustic wave, namely $0.5 \%$ of $U_{0}$ (circles), $2.5 \%$ (pluses), $3.5 \%$ (crosses) and $4.5 \%$ (squares). After [112].

(13) The power spectrum of the velocity pulsations in response to low-frequency acoustic forcing of a circular jet for $\mathrm{St}_{\mathrm{a}}=0.25, x / D=0.5$. After [50].

(14) The evolution of power spectra of the velocity pulsations with increasing relative distance $x / D$ from the nozzle exit under high-frequency acoustic forcing at $\mathrm{St}_{\mathrm{a}}=2.5$. After [50].

(15) The dependence on distance from the nozzle of the Strouhal number corresponding to the spectral line of highest intensity, in the presence of high-frequency acoustic forcing for Strouhal number 3.54, along the jet axis (light circles) and along a line offset by $R$ from the axis (filled circles). After [113]. 
(16) Numerical simulations showing the pendulum oscillations (lower plot in each case) and their power spectra (upper plots) with increasing noise intensity for $\omega_{0}=1$, $\beta=0.1, \alpha=100$ and: (a) $\kappa\left(2 \omega_{0}\right) / \kappa_{c r}=1.01 ;(b) \kappa\left(2 \omega_{0}\right) / \kappa_{c r}=1.56 ;(c) \kappa\left(2 \omega_{0}\right) / \kappa_{c r}=$ 2.44: and $(d) \kappa\left(2 \omega_{0}\right) / \kappa_{c r}=6.25$. After [45].

(17) The dependence of $\sigma={\overline{\varphi^{2}}}^{1 / 2}$ on $\omega_{a}$ for $\omega_{0}=1, \beta=0.1, \alpha=100, \kappa\left(2 \omega_{0}\right) / \kappa_{c r}=1.01$, $a=0.5$. After [45].

(18) The dependences of $\sigma$ on the amplitude $a$ of low-frequency vibration for $\omega_{0}=1$, $\beta=0.1, \alpha=100$ and: (a) $\kappa\left(2 \omega_{0}\right) / \kappa_{c r}=1.89, \omega_{a}=0.3 ;(b) \kappa\left(2 \omega_{0}\right) / \kappa_{c r}=2.23$, $\omega_{a}=1.5$. After [45].

(19) Time series of $\varphi(t)$ and $\dot{\varphi}(t)$ for $\omega_{0}=1, \beta=0.1, \alpha=100, \kappa\left(2 \omega_{0}\right) / \kappa_{c r}=5.6$, $\omega_{a}=19.757$ and: (a) $a=5$; (b) $a=15$; (c) $a=30$; (d) $a=40$. After [45].

(20) The dependences of $\sigma$ on $a$ for $\omega_{0}=1, \beta=0.1, \alpha=100, \kappa\left(2 \omega_{0}\right) / \kappa_{c r}=5.6$ and: (a) $\omega_{a}=3.5 ;(b) \omega_{a}=6 ;(c) \omega_{a}=11 ;(d) \omega_{a}=19.757$. After [45].

(21) The dependence on $\omega_{a}$ of $\sigma / \sigma_{0}$, where $\sigma_{0}$ is the value of $\sigma$ in the absence of additional vibration, for $\kappa\left(2 \omega_{0}\right) / \kappa_{c r}=5.6, a=2.5$ (filled circles), $a=5$ (pluses), $a=10$ (squares), and $a=20$ (crosses). After [45].

(22) Time series $\varphi(t)$ and $\dot{\varphi}(t)$ in the presence of additive noise in Eq. (4.1) with multiplicative noise $\xi(t)$ of variance 0.05 for: (a) $a=40$; and (b) $a=50$. The other parameters are the same as in Fig. 20. After [45].

(23) Plots of various quantities versus $y$ for $b_{0}=0.1, q=3, r_{0}=0.5, \operatorname{Re}=25000$ : (a) $u_{\mathrm{d}}(0, y) ;(b) u_{\mathrm{d}}(8, y) ;(c) v_{\mathrm{d}}(0, y) ;(d) v_{\mathrm{d}}(8, y) ;(e) \Omega_{\mathrm{d}}(0, y) ;$ and $(f) \Omega_{\mathrm{d}}(8, y)$.

(24) The dependences on the Strouhal number St for $b_{0}=0.1, q=3, r_{0}=0.5, \operatorname{Re}=25000$ and different $x$ of: (a) the gain factor $\Gamma_{0}$ and (b) the wave phase velocity $v_{\mathrm{ph} 0}=\mathrm{S} / K_{0}$. The value of $x$ is indicated near the corresponding curve in each case.

(25) The dependences on the Strouhal number St of (a) the gain factor $\Gamma_{0}$ and $(b)$ the wave phase velocity $v_{\mathrm{ph} 0}=\mathrm{S} / K_{0}$ for $x=0$ and $\operatorname{Re}=25000, b_{0}=0.1$ (curve 1 ), $\operatorname{Re}=100000, b_{0}=0.05$ (curve 2 ) and $\operatorname{Re}=100000, b_{0}=0.02$ (curve 3 ).

(26) The partial solutions of Eq. (6.31) for ( $a$ and $b) y_{1} \leq y \leq 1$ and ( $c$ and $d$ ) $1 \leq y \leq y_{2}$ : $\operatorname{Re}=25000, b_{0}=0.1 \mathrm{~m} x=0, \mathrm{~S}=22\left(Q_{0} \approx 44.357517+0.597408 i\right)$. It is found that ( $a$ and $b$ ) all solutions tend to $B_{3}$, and ( $c$ and $d$ ) that the first solution tends to the second one. 
(27) The partial solutions of Eqs. (6.31) and (6.39) for ( $a$ and $b) y_{1} \leq y \leq 1$ and ( $c$ and d) $1 \leq y \leq y_{2}$. The parameters are the same as in Fig. 25. It is seen that all solutions are stable.

(28) Plots of the real and imaginary parts and modulus of the eigenfunction $f_{0}$, calculated for $b_{0}=0.1, q=3, \operatorname{Re}=25000, x=0$ and $\left(a S=10\left(Q_{0} \approx 19.952633+9.984723 i\right)\right.$ and $(b) \mathrm{S}=18\left(Q_{0} \approx 37.974133+4.244753 i\right)$.

(29) Plots of the real and imaginary parts and modulus of the adjoint eigenfunction $\bar{\chi}_{0}$, calculated for $b_{0}=0.1, q=3, \operatorname{Re}=25000, x=0$ and $\left(\mathrm{a} S=10\left(Q_{0} \approx 19.952633+\right.\right.$ $9.984723 i)$ and $(b) \mathrm{S}=18\left(Q_{0} \approx 37.974133+4.244753 i\right)$.

(30) The first partial solution of Eq. (6.39) $B_{1}(\mathrm{~S}, x, y)$ versus $y$ over the range $y_{1} \leq y \leq y_{2}$ for $x=0, \mathrm{~S}=13$.

(31) The eigenfunctions and adjoint eigenfunctions versus $y$ over the range $y_{1} \leq y \leq y_{2}$ for $x=0, \mathrm{~S}=1$ (bold lines), and the same quantities calculated from the Euler equations (thin lines).

(32) The dependences on the Strouhal number St for $b_{0}=0.1, q=3, r_{0}=0.5, \operatorname{Re}=25000$ and different $x$ of: (a) $\Gamma=\Gamma_{0}+\Gamma_{1} / \lambda$ and (b) the wave phase velocity $v_{\mathrm{ph}}=\mathrm{S} /\left(K_{0}+\right.$ $\left.K_{1} / \lambda\right)$. The value of $x$ is indicated near the corresponding curve in each case.

(33) The dependences of (a) $\partial \Gamma_{0} / \partial x \equiv \Gamma_{0 x}$ and (b) $\partial v_{\mathrm{ph} 0} / \partial x \equiv v_{0 x}$ on the Strouhal number St for $x=0,0.5,1,2,3,5$ and 8 .

(34) Plot of $\kappa(\mathrm{S}, 0,0) / \kappa_{0}$ described by $(6.47)$.

(35) Evolution of $\kappa(\mathrm{S}, x, y) / \kappa_{0}$ in the linear approximation for (a) $y=0$ and $(b)|y|=0.7$.

(36) The dependences of the Strouhal number $\mathrm{St}_{\mathrm{m}}$ on the distance from the nozzle for a $y=0$ and $b|y|=0.7$ (the linear (zeroth) approximation). The dependences $\mathrm{St}_{\mathrm{m}} \approx 3.2 x^{-0.36}$ (for $y=0$ ) and $\mathrm{St}_{\mathrm{m}} \approx 6.5 x^{-0.68}$ (for $y=0.7$ ) are shown by solid lines.

(37) Plots of the mean-root-square values of turbulent velocity pulsations versus $x$ for $(a)$ $y=0\left(\epsilon_{u}(x, 0)\right)$ and $(b) y=0.7\left(\epsilon_{u}(x, 0.7)\right)$.

(38) The changes of $(a, c)$ the group wave velocity at $\mathrm{St}=\mathrm{St}_{\mathrm{m}}$ and $(b, d)$ the wave lengths in longitudinal (1) and transverse (2) directions $\left(\lambda_{\mathrm{lon}}\left(\mathrm{St}_{\mathrm{m}}, x\right)=2 \pi / K\left(\mathrm{St}_{\mathrm{m}}, x\right)\right.$ and $\lambda_{\mathrm{tr}}\left(\mathrm{St}_{\mathrm{m}}, x\right)=2 \pi / \Gamma\left(\mathrm{St}_{\mathrm{m}}, x\right)$, respectively), with increasing $x:(a, b)$ for $y=0 ;(c, d)$ for $y=0.7$. 
(39) The dependences of the mean velocity $\langle u(x, y)\rangle=u_{\mathrm{d}}(x, y)+\left\langle q_{1}(x, y)\right\rangle$ taking account of the correction caused by the turbulent pulsations $\left(\left\langle q_{1}(x, y)\right\rangle\right)($ a) on $y$ at $x=6$ (1), $x=7(2)$ and $x=8(3)$ and (b) on $x$ at (from right to left) $y==0,0.1,0.2,0.3$, $0.4,0.5,0.6$ and 0.7 .

(40) (a) An example of the velocity profile taking account of the stochastic constituents (curve 1) and the corresponding profile of the dynamical constituent of the velocity (curve 2) for $x=8$ (the end of initial part); and (b) the internal (1) and external (2) boundaries of the mixing layer taking account of the stochastic constituents.

(41) Examples of the dependences of $\Delta \kappa$ on the Strouhal number St for (a) $y=0.7$ and a number values of $x$, and $(b)$ for $x=8$ and three values of $y=0.01$ (curve 1 ), 0.5 (2) and $0.7(3)$. Because $\Delta \kappa$ changes strongly as the values of $x$ and $y$ vary, we have plotted not $\Delta \kappa$ but $(\Delta \kappa)^{1 / 15}$.

(42) Examples of the velocity pulsations spectral density with taking into account of the nonlinearity: $x=6$, (curve 1) $y=0.5$ and (curve 2) $y=0.7$. For comparison in the same figure are given the corresponding dependences found in the linear approximation (curves 3 and 4 , respectively). 

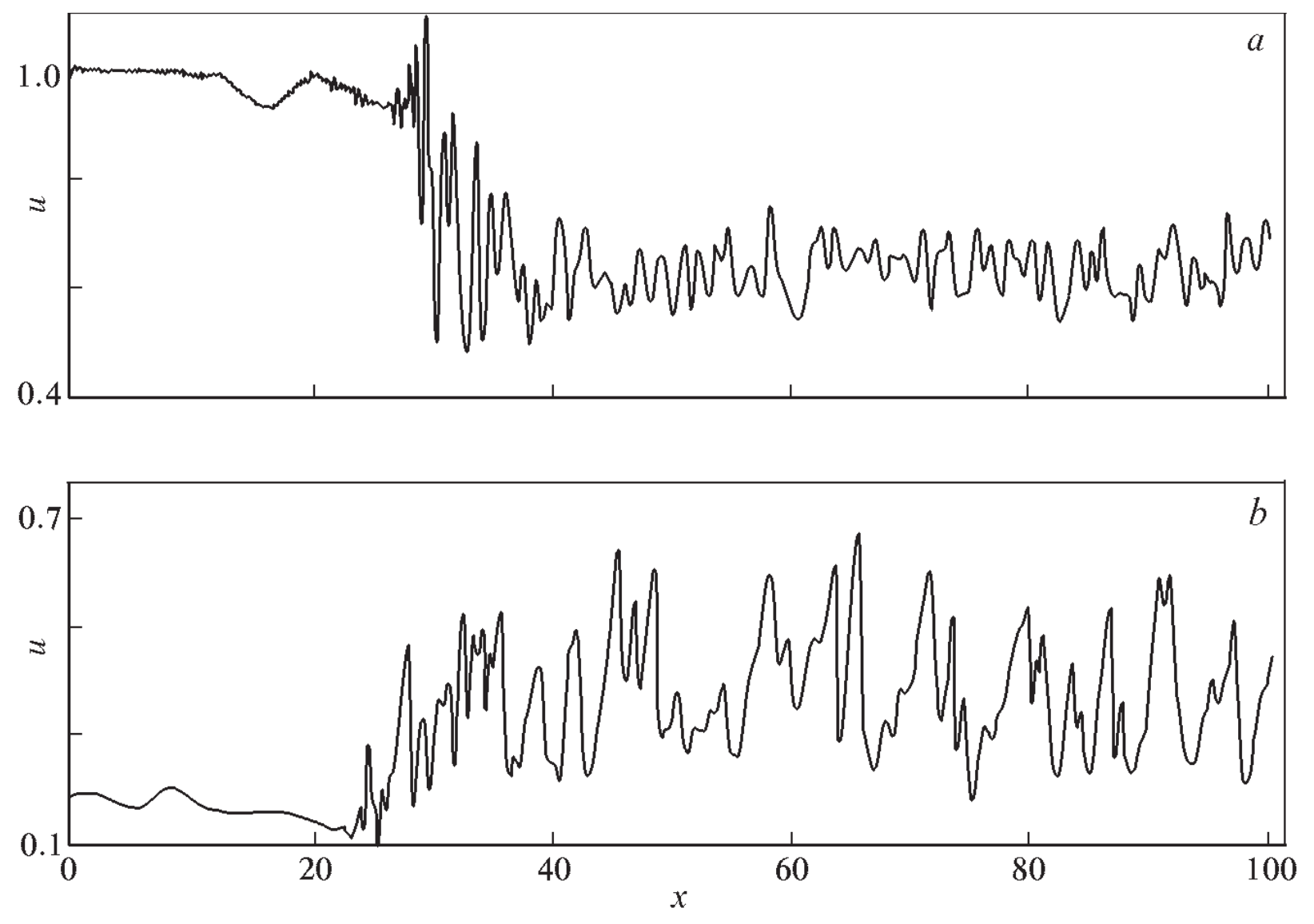

Fig. 1.
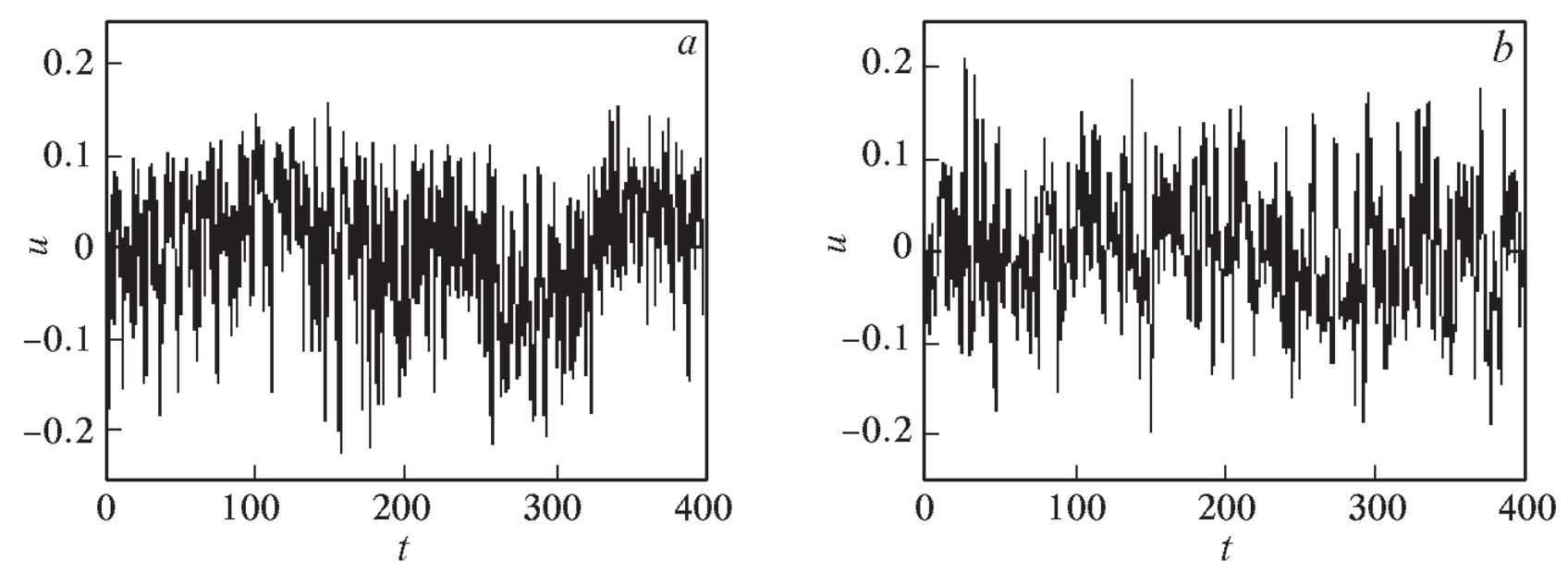

Fig. 2. 


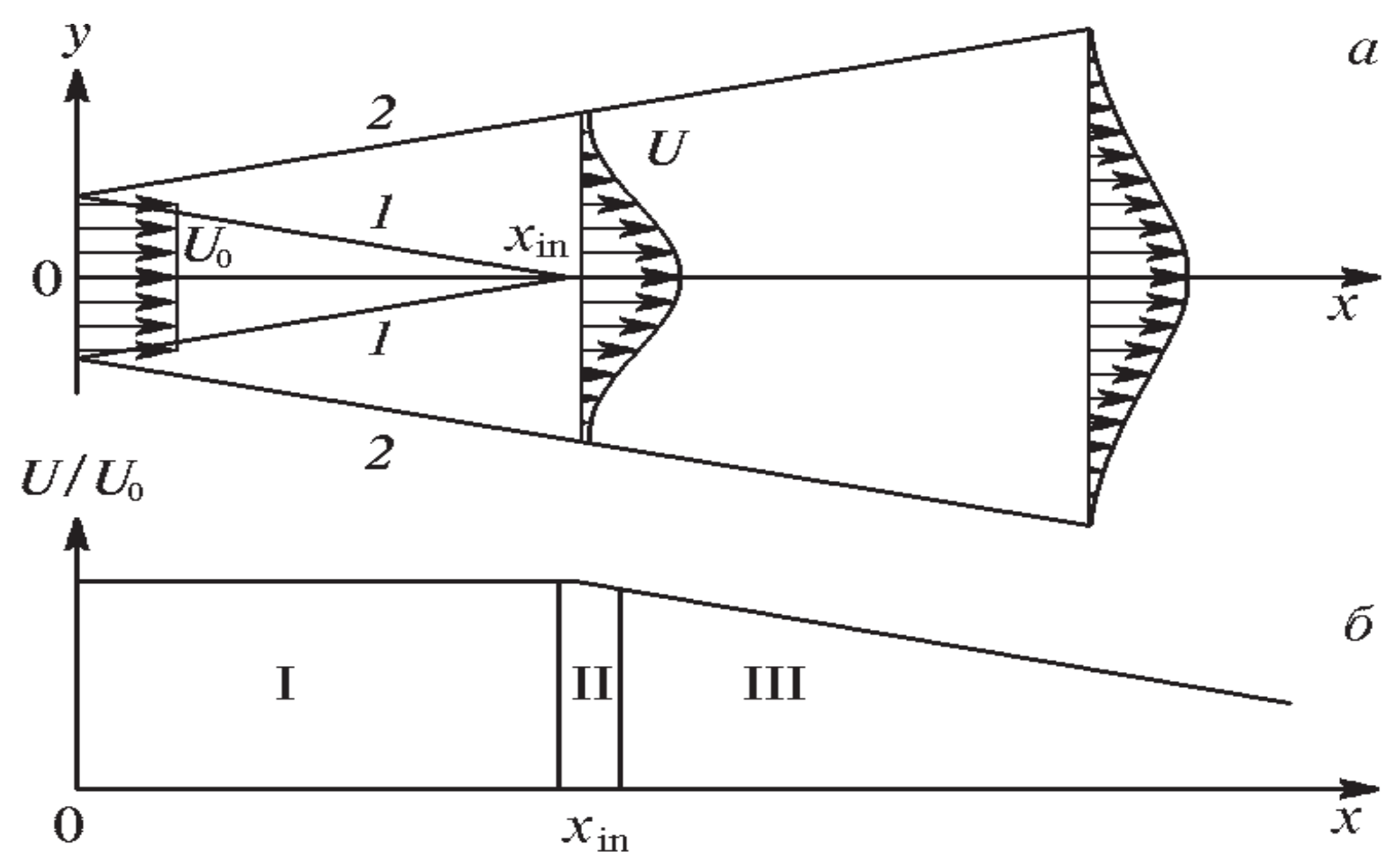

Fig. 3.

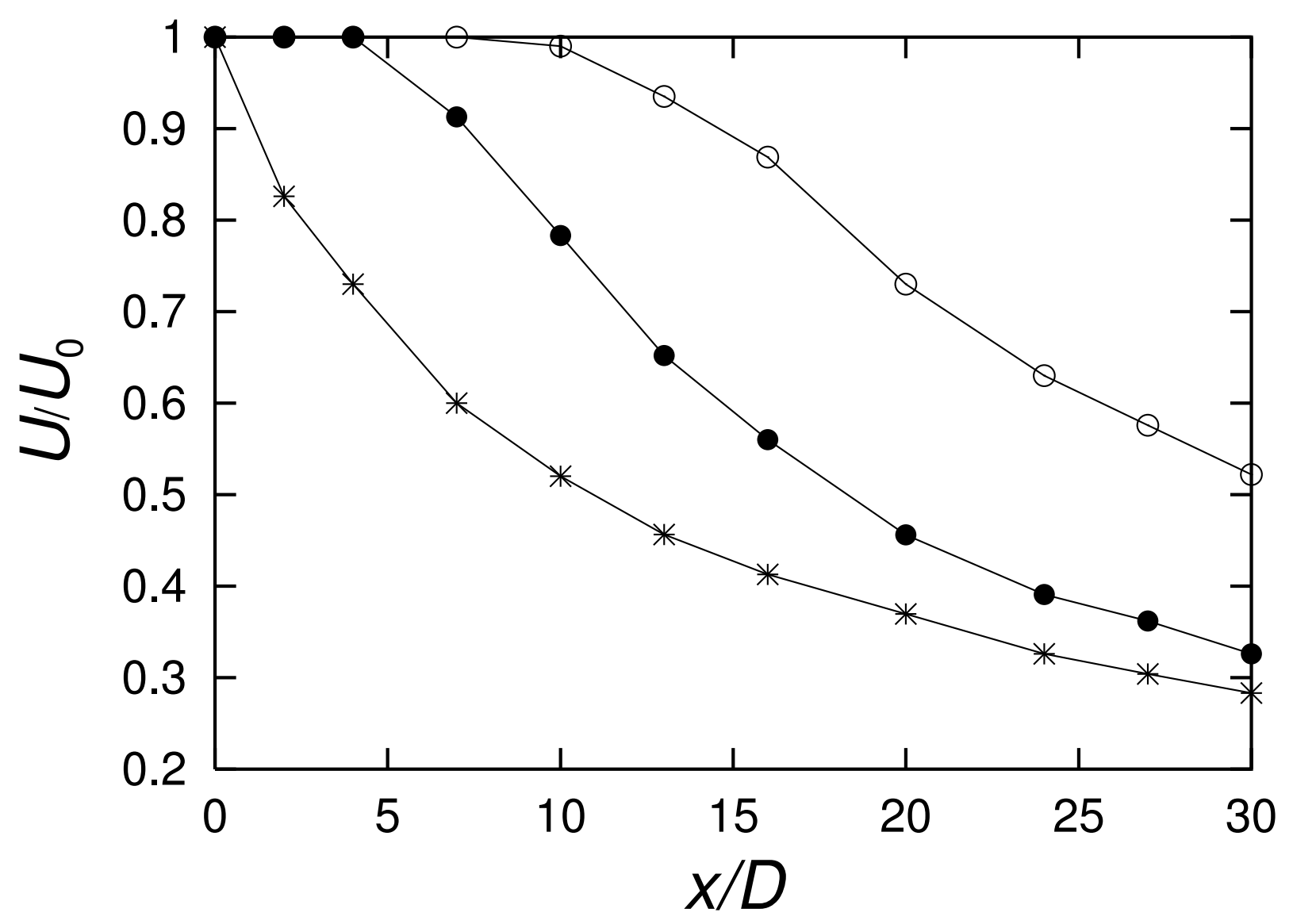

Fig. 4. 

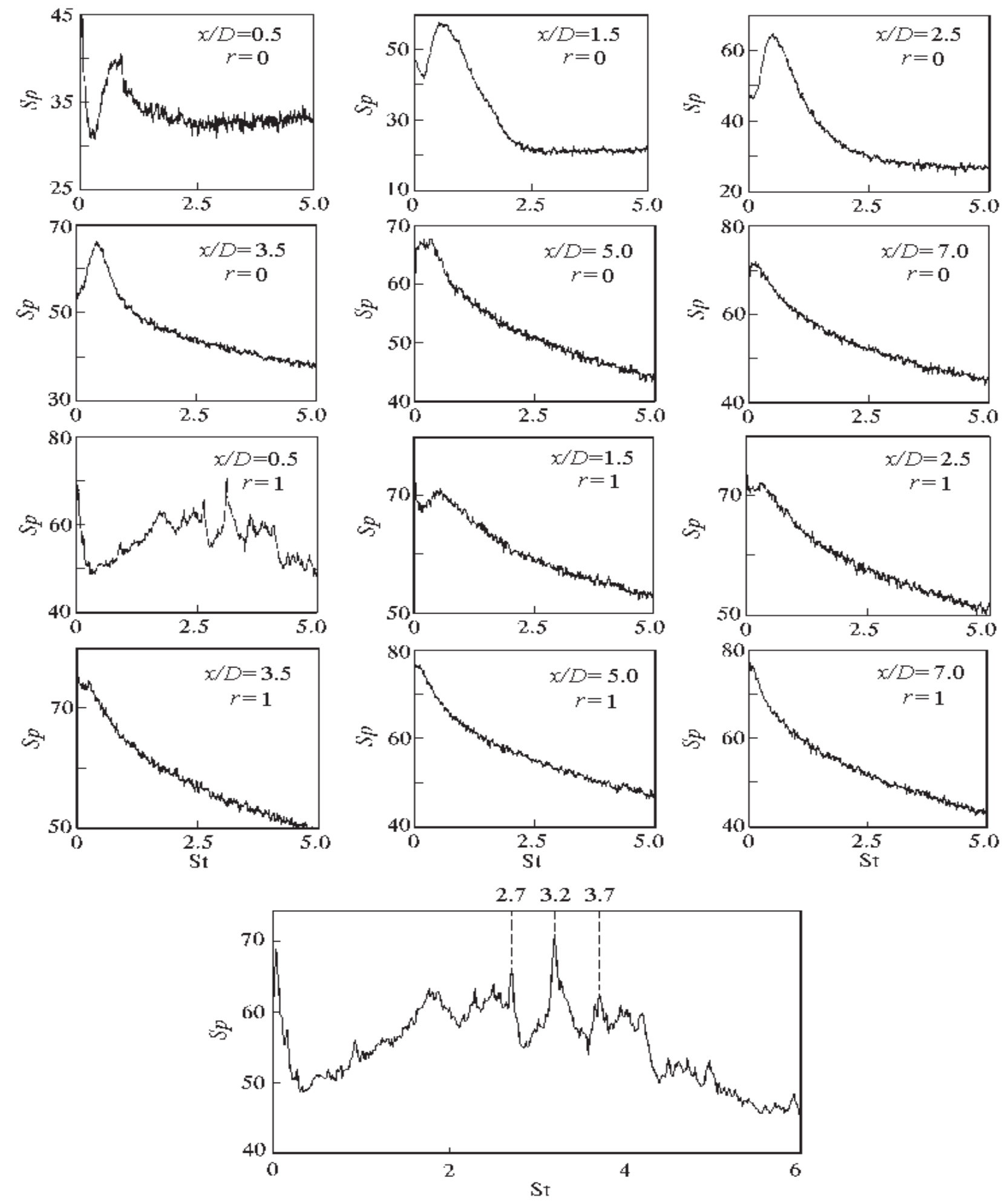

Fig. 5. 

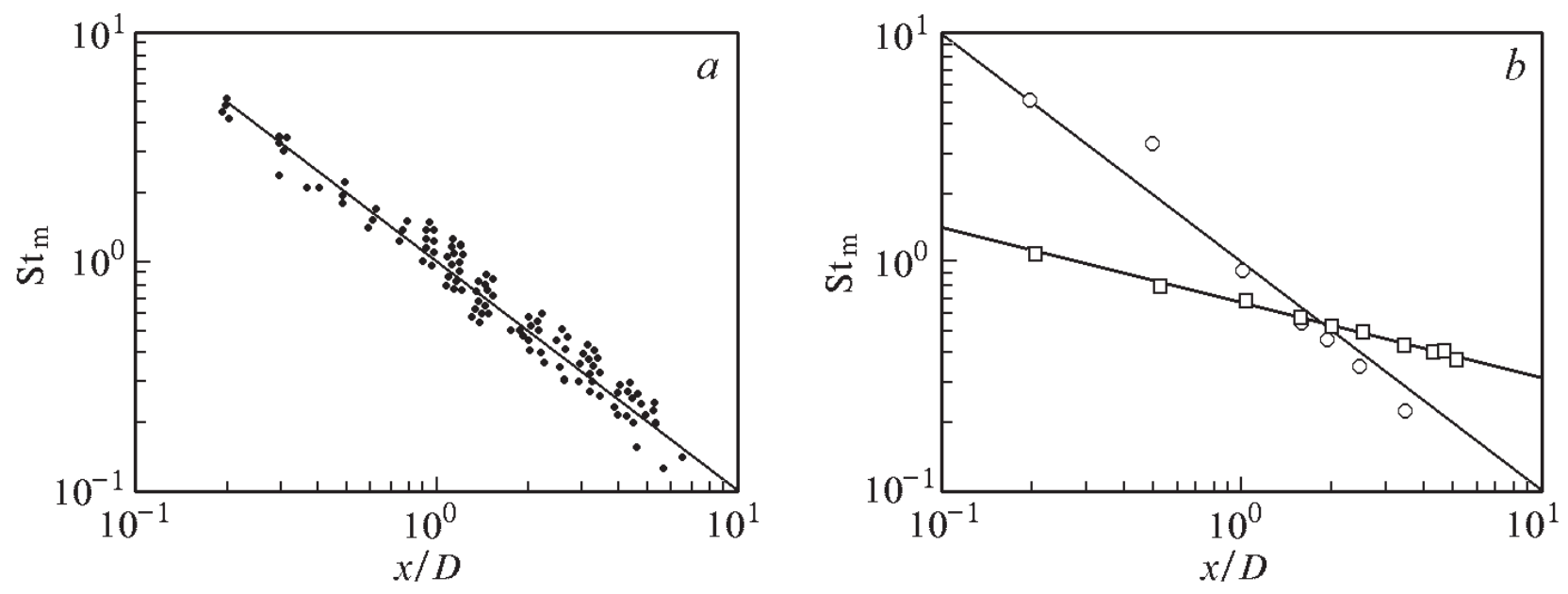

Fig. 6.
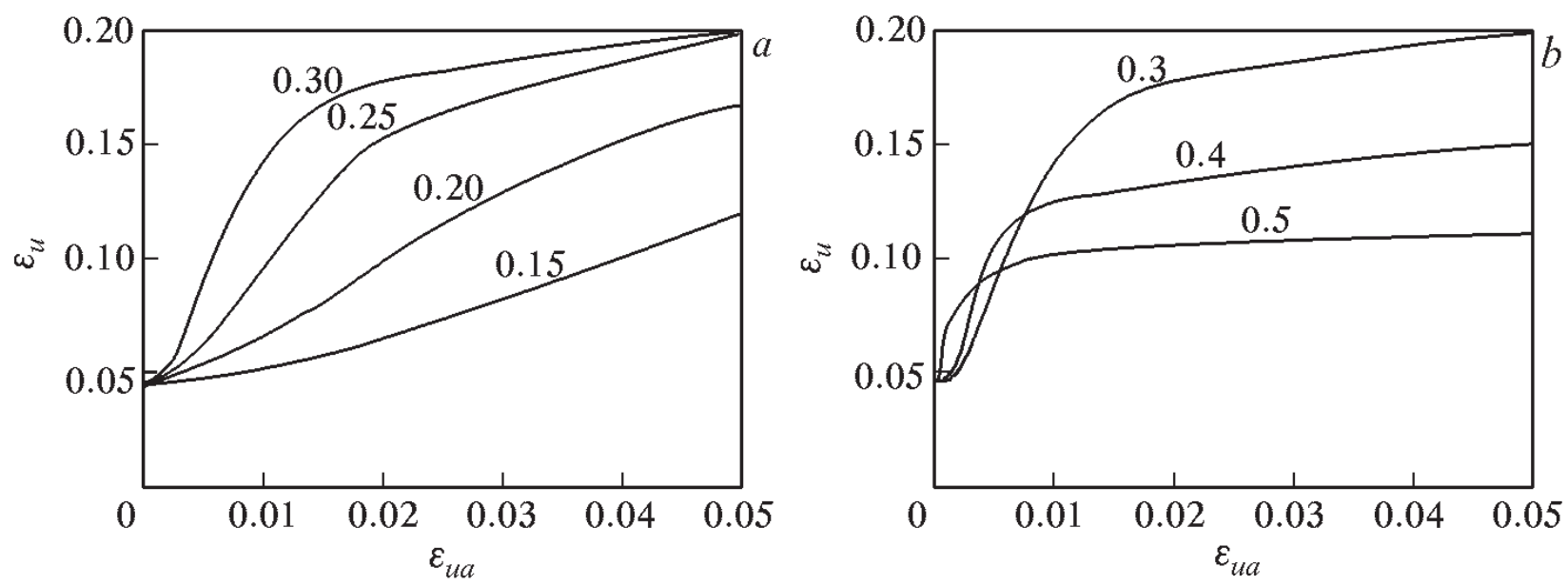

Fig. 7. 


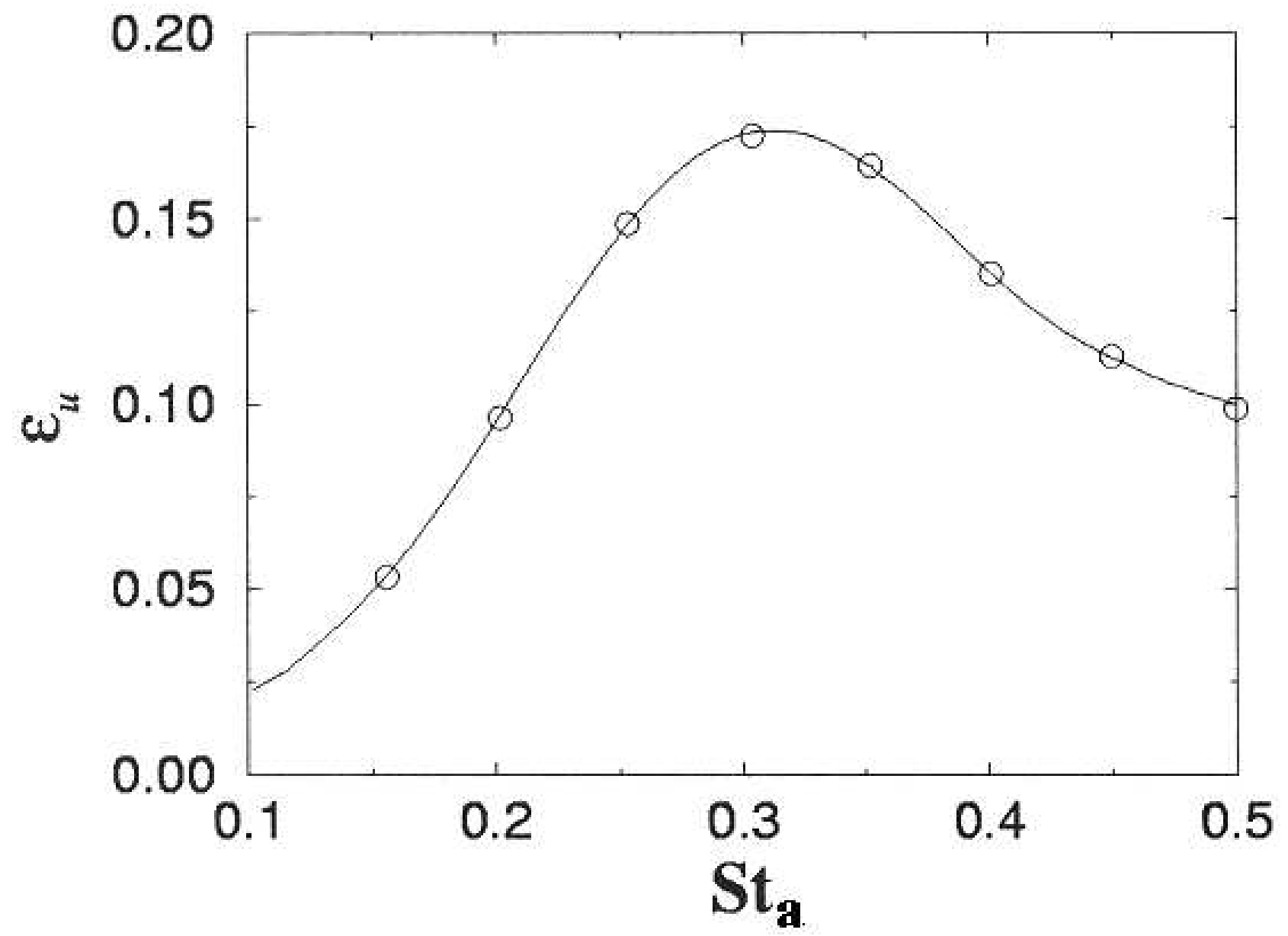

Fig. 8.

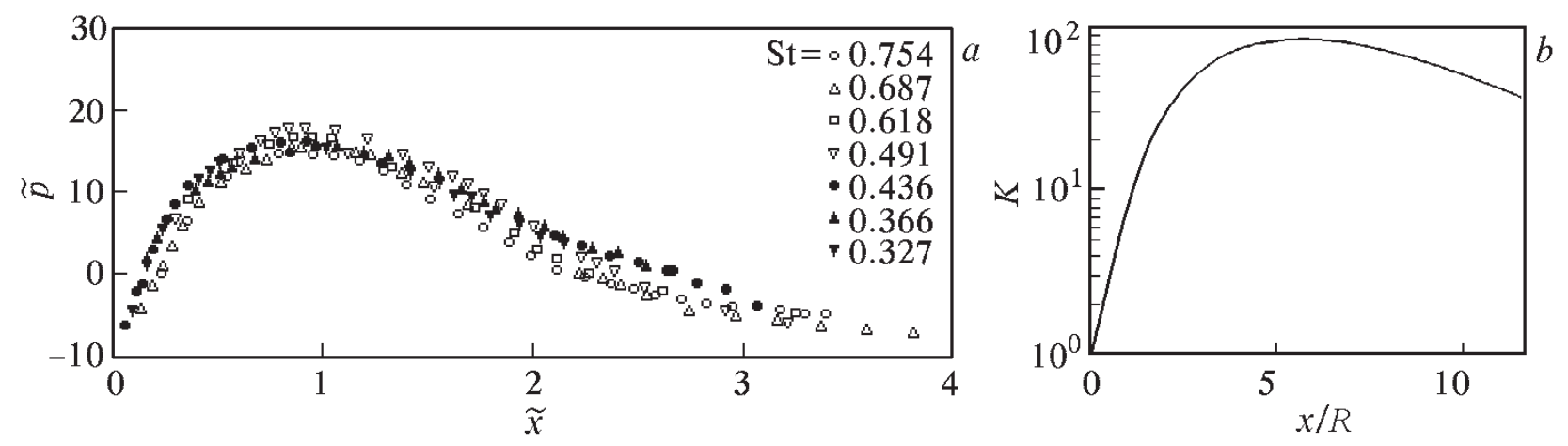

Fig. 9. 

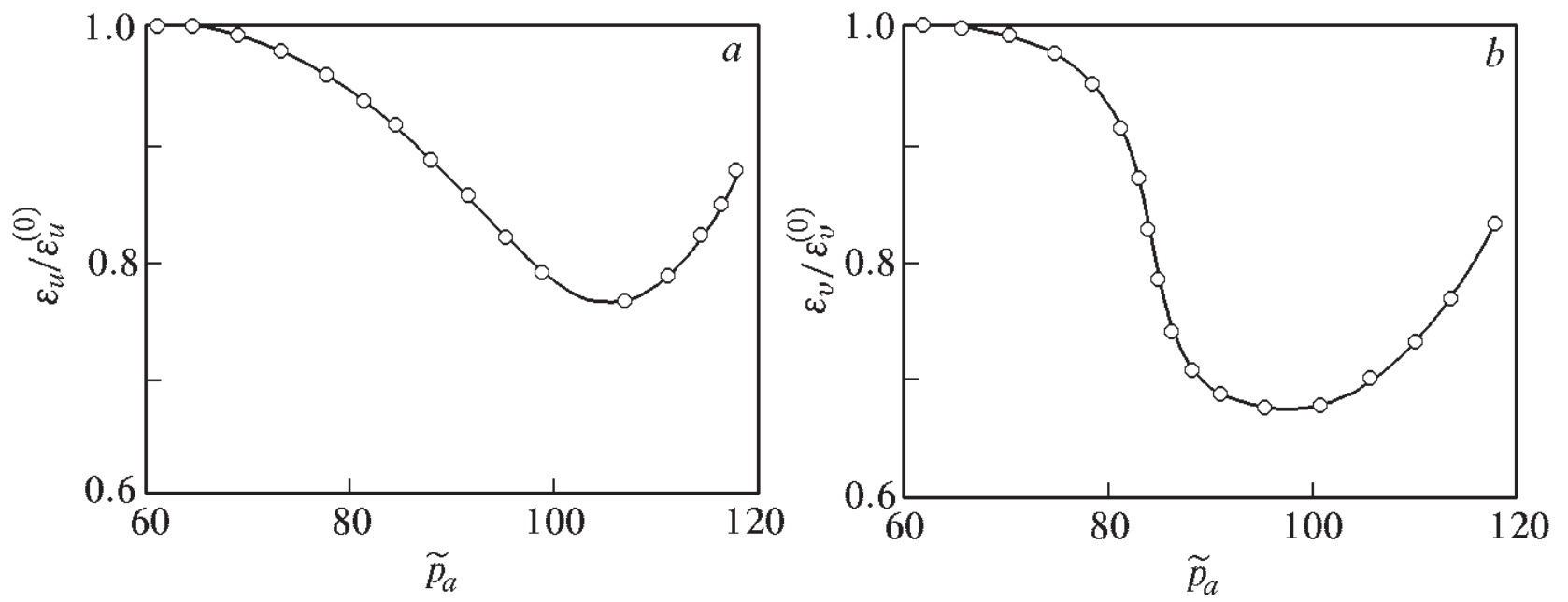

Fig. 10. 


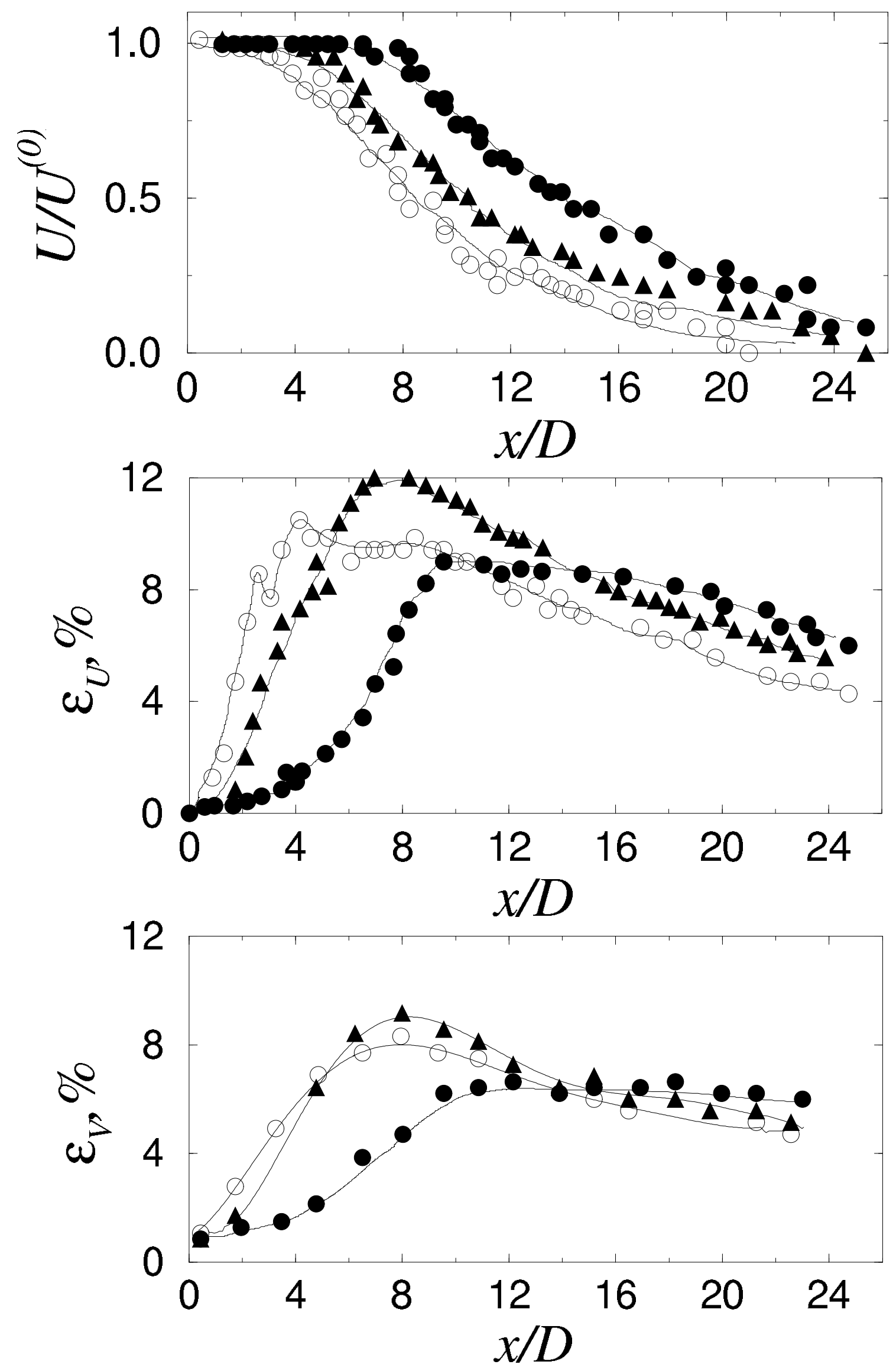

Fig. 11. 


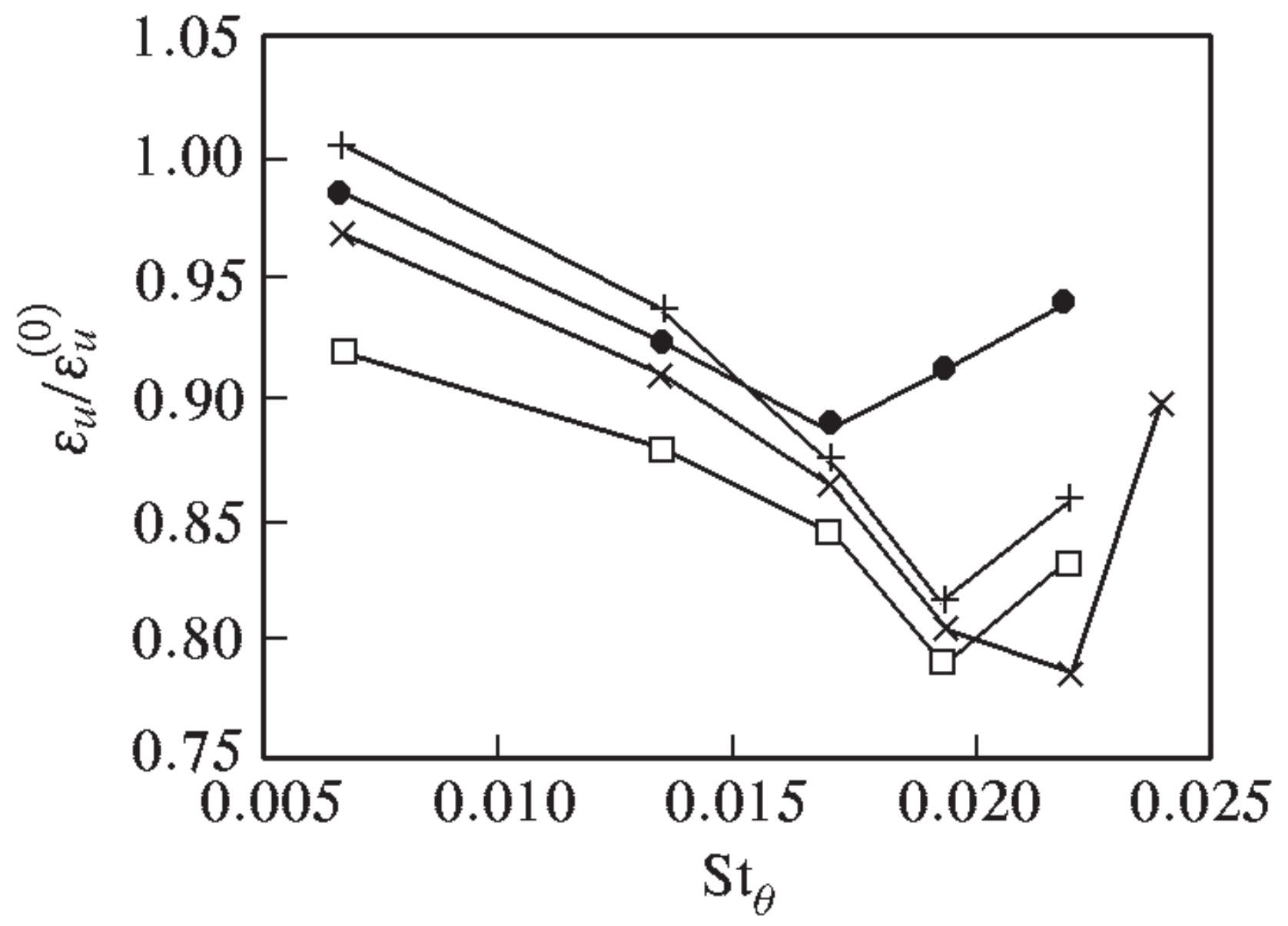

Fig. 12 . 


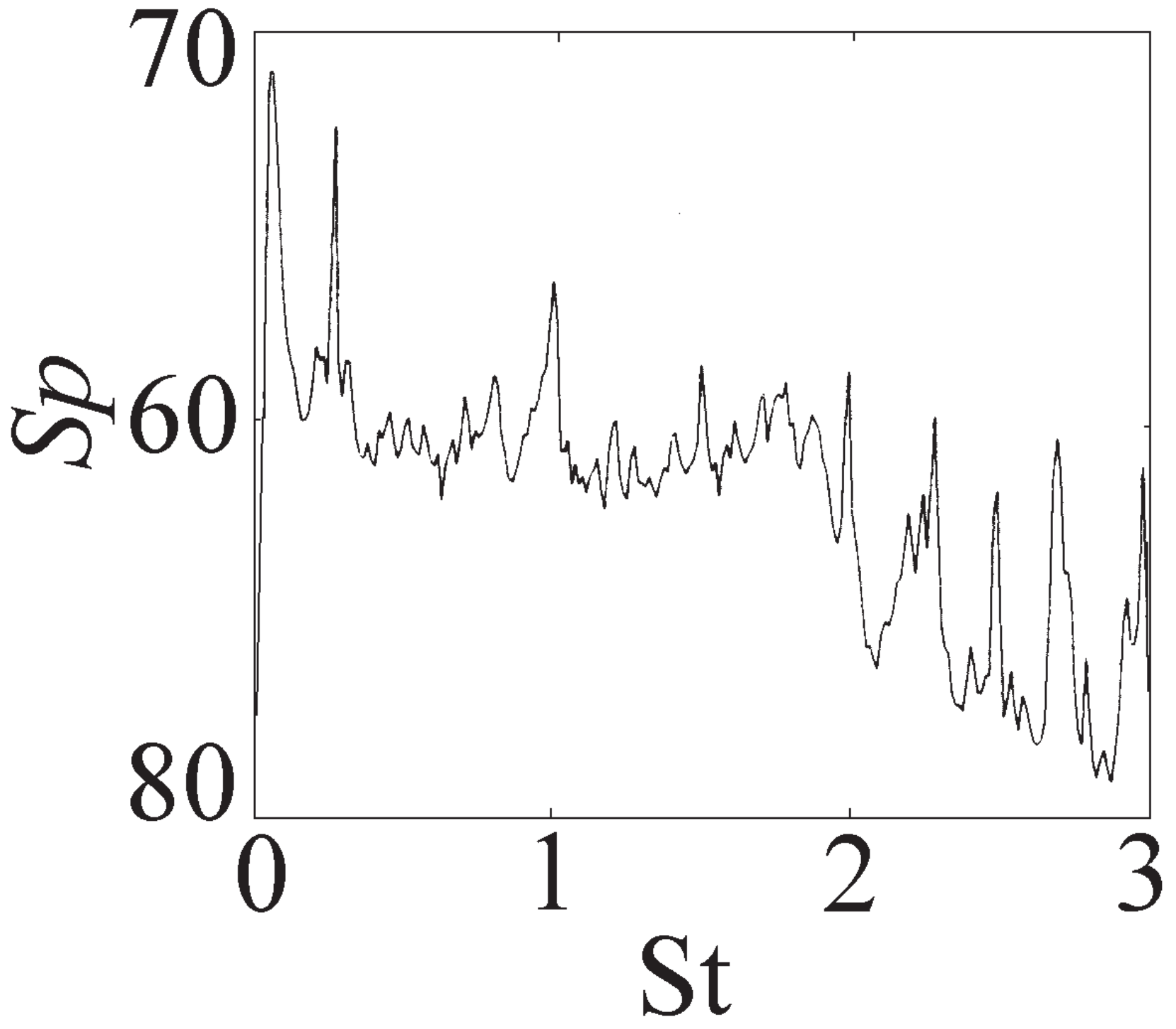

Fig. 13. 

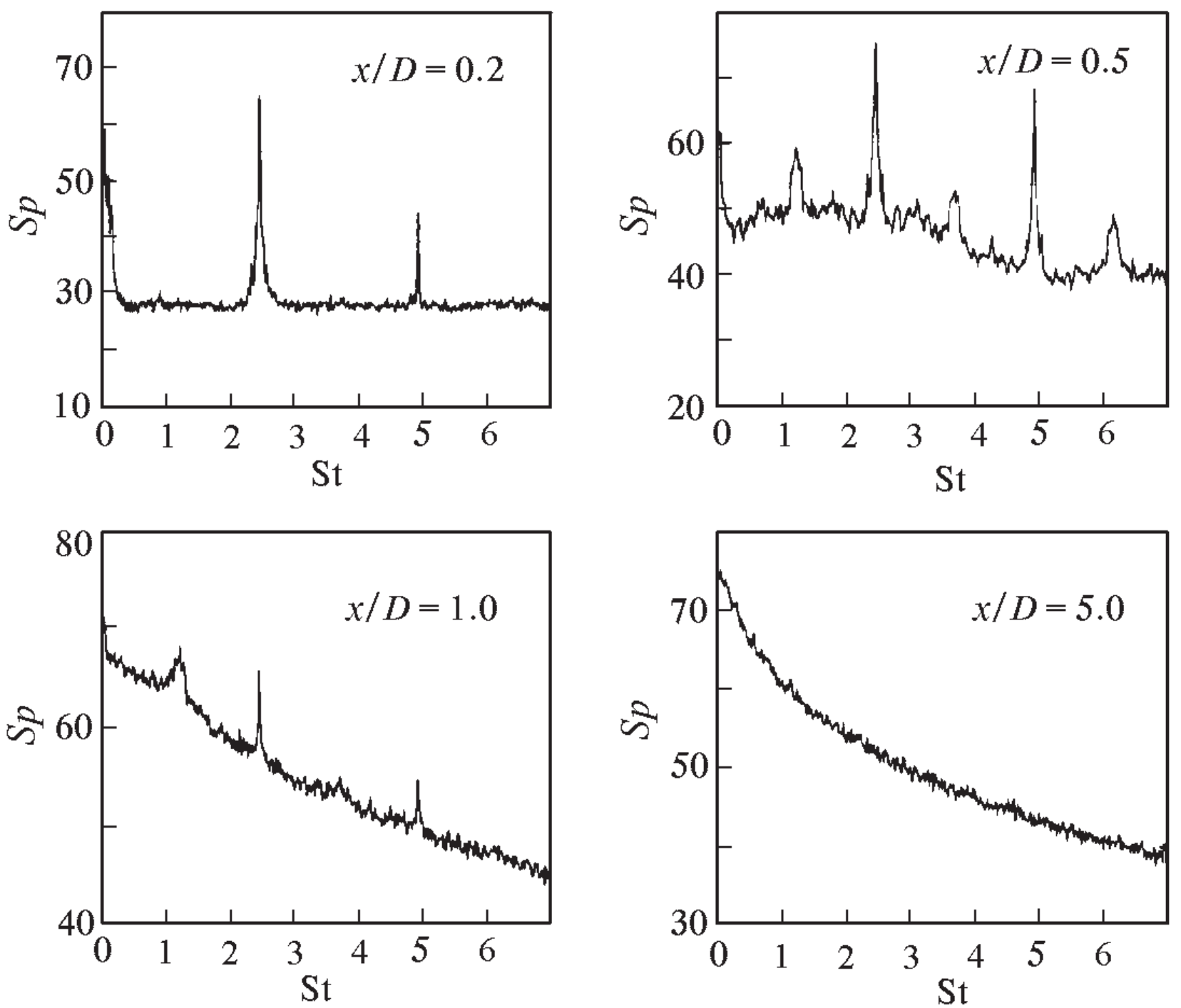

Fig. 14. 


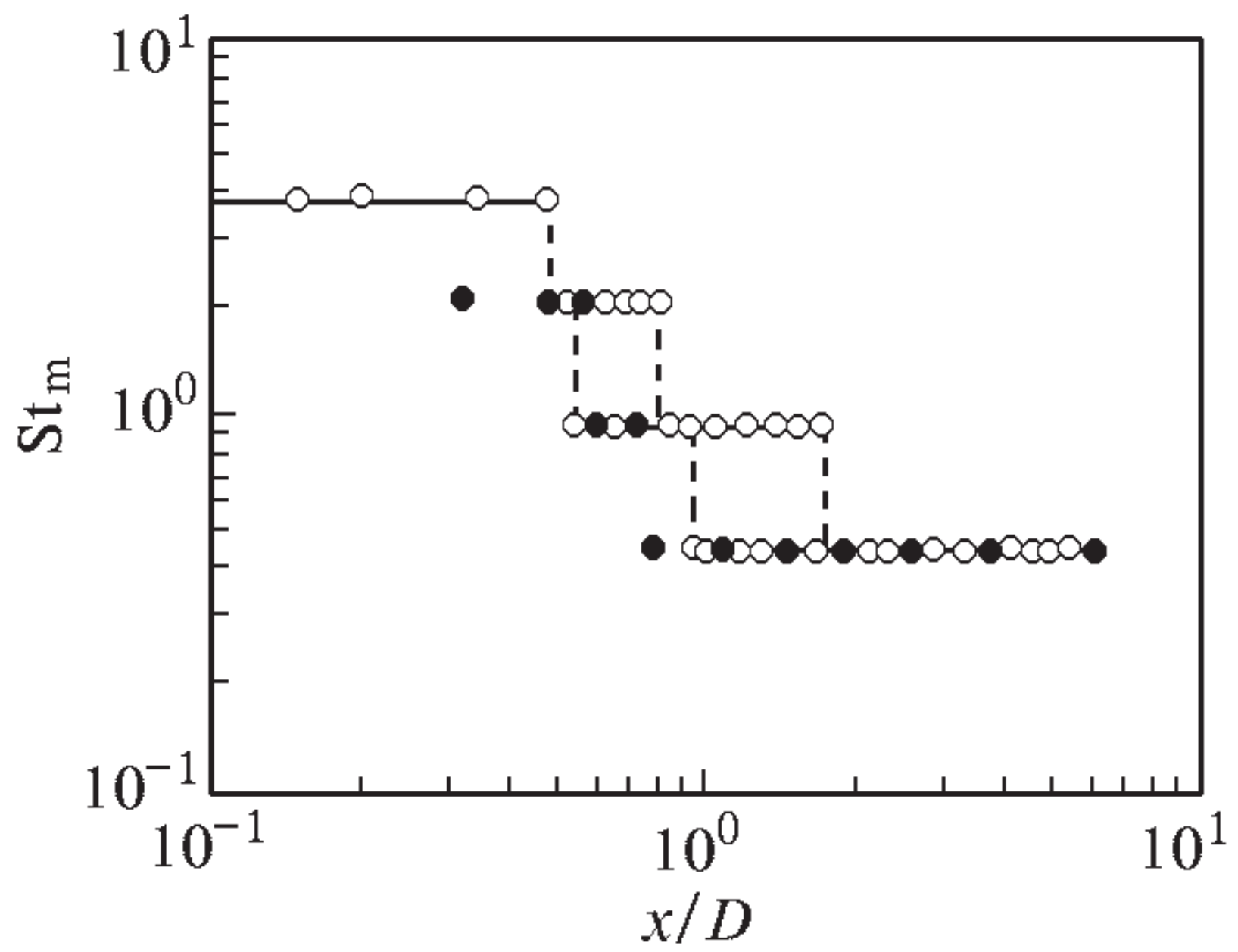

Fig. 15 . 

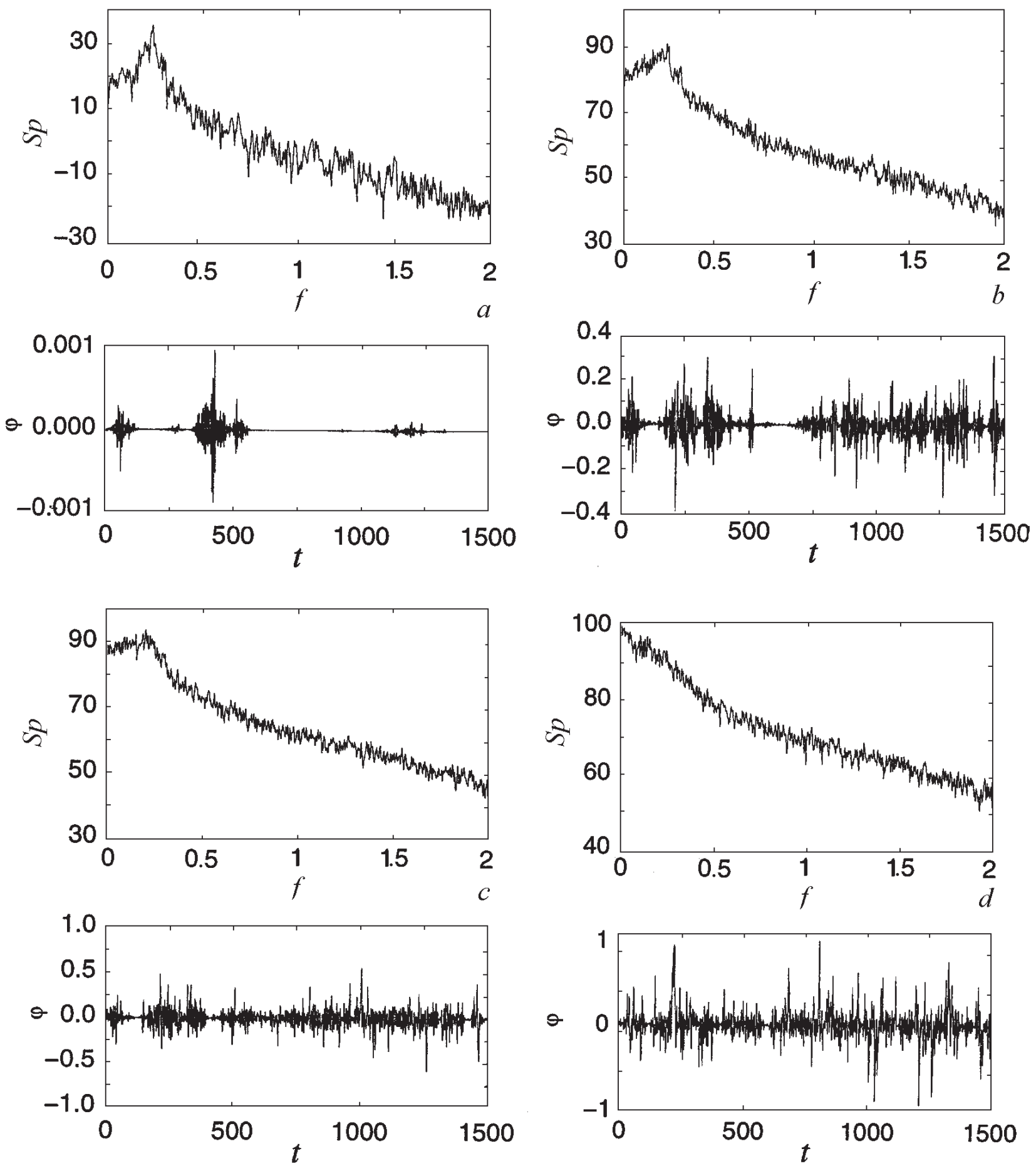

Fig. 16. 


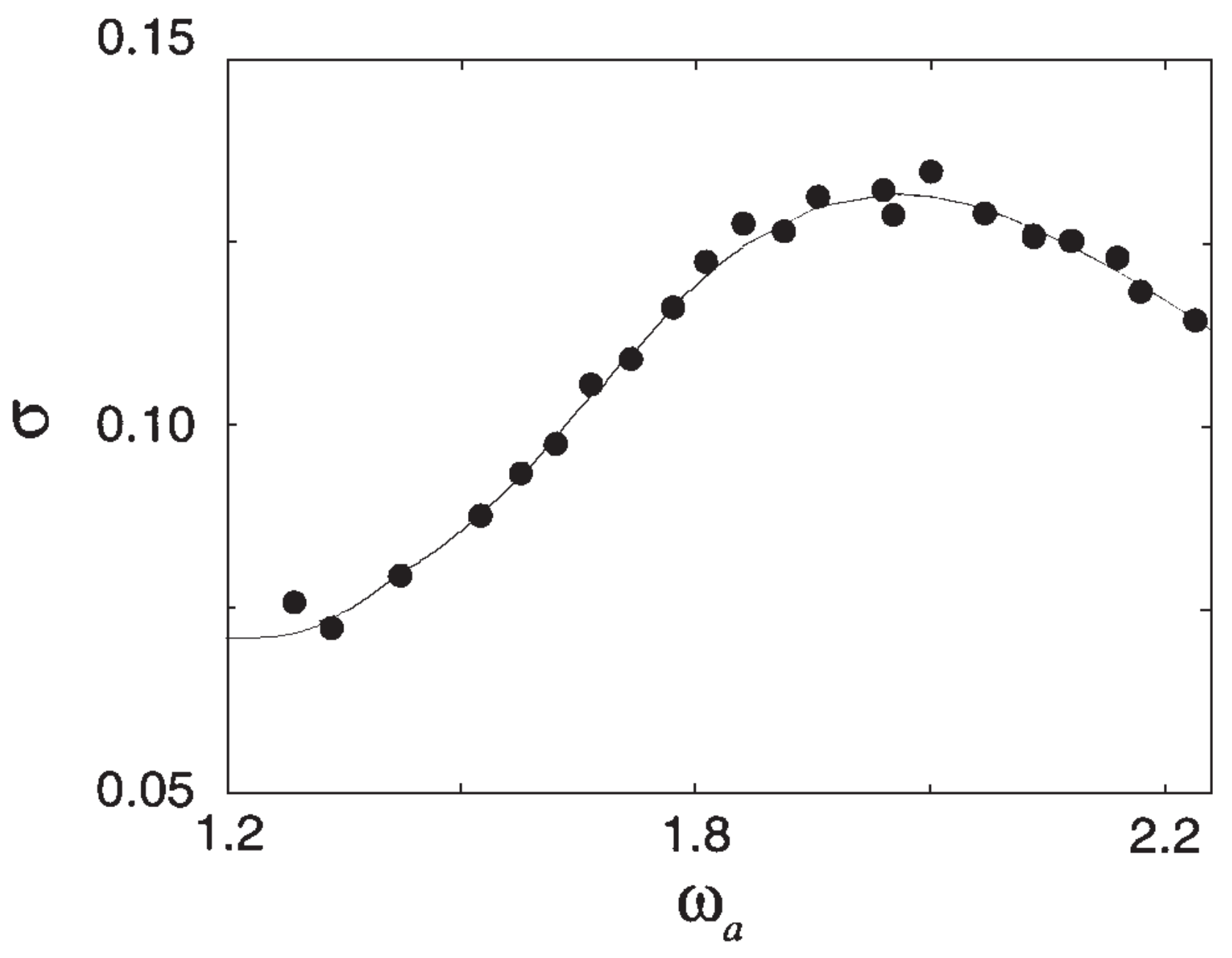

Fig. 17. 

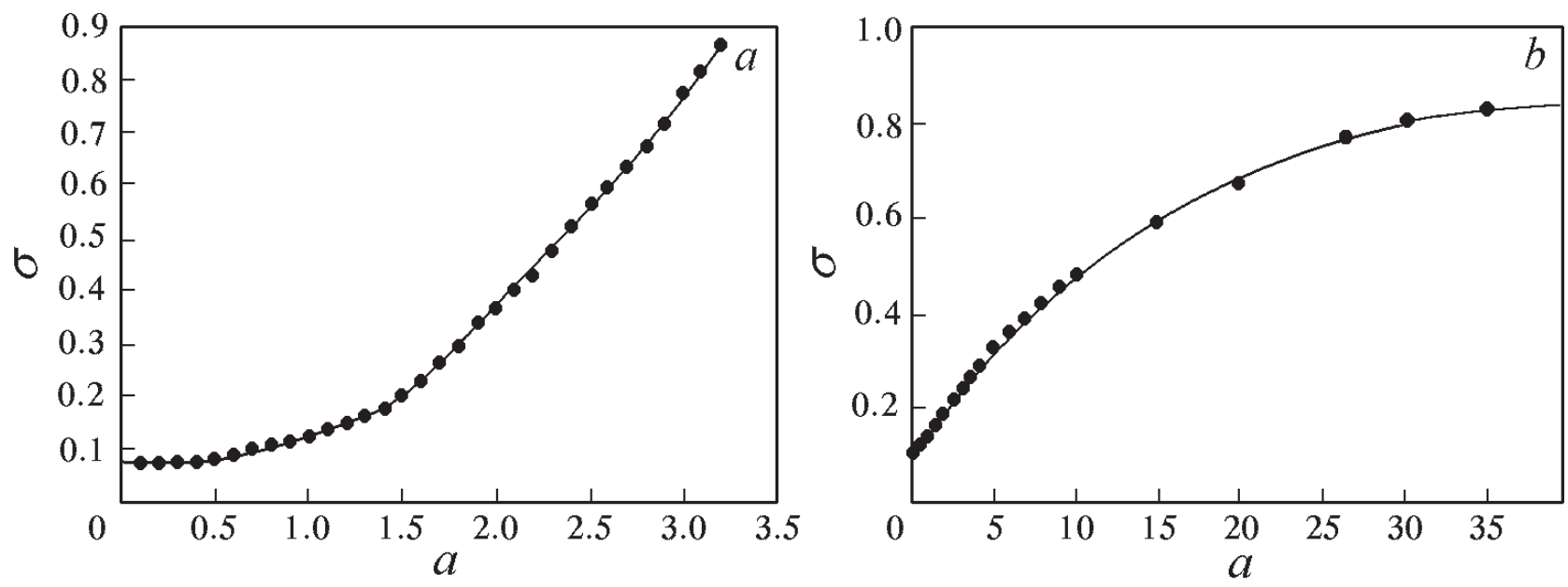

Fig. 18 .
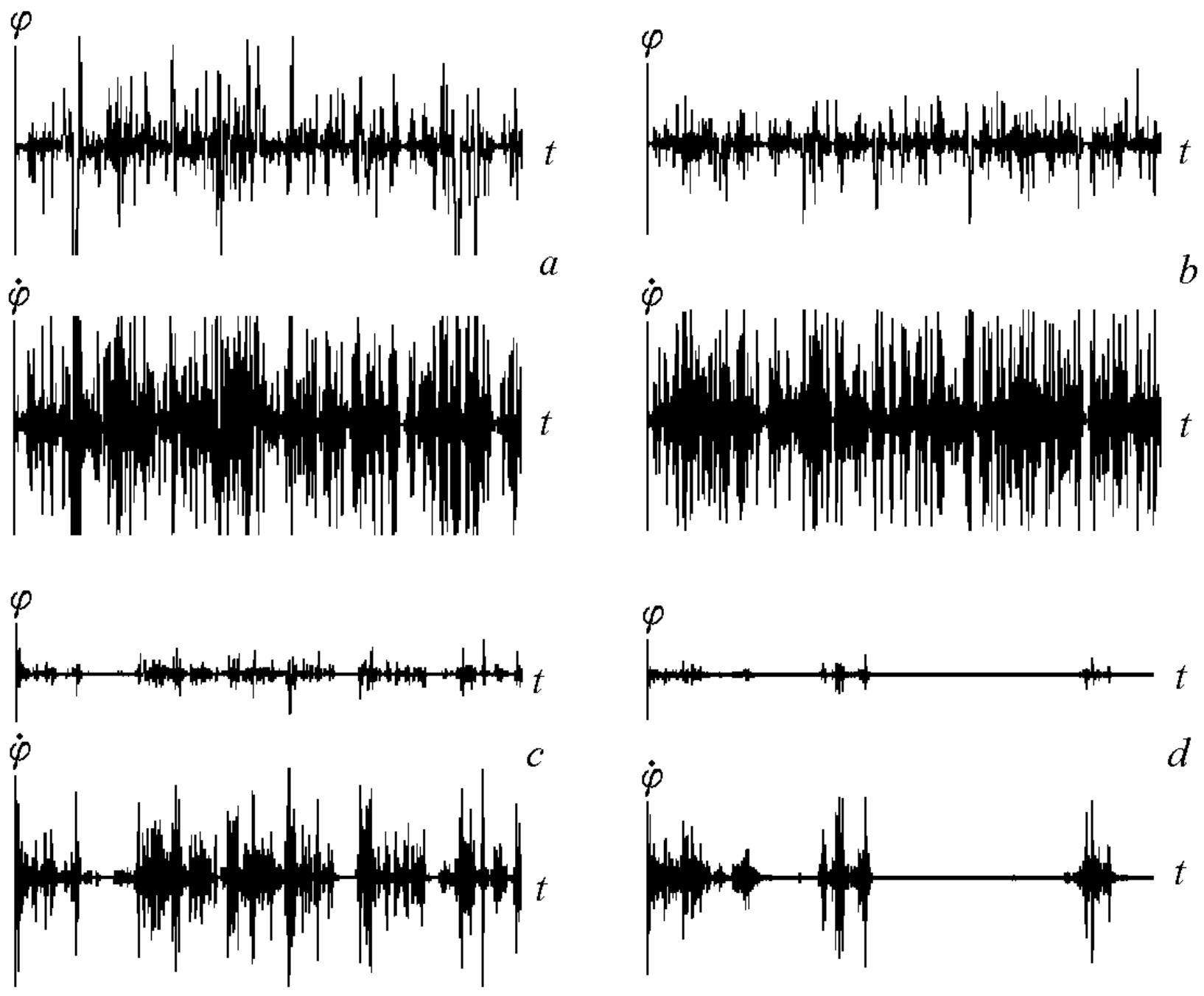

Fig. 19 . 

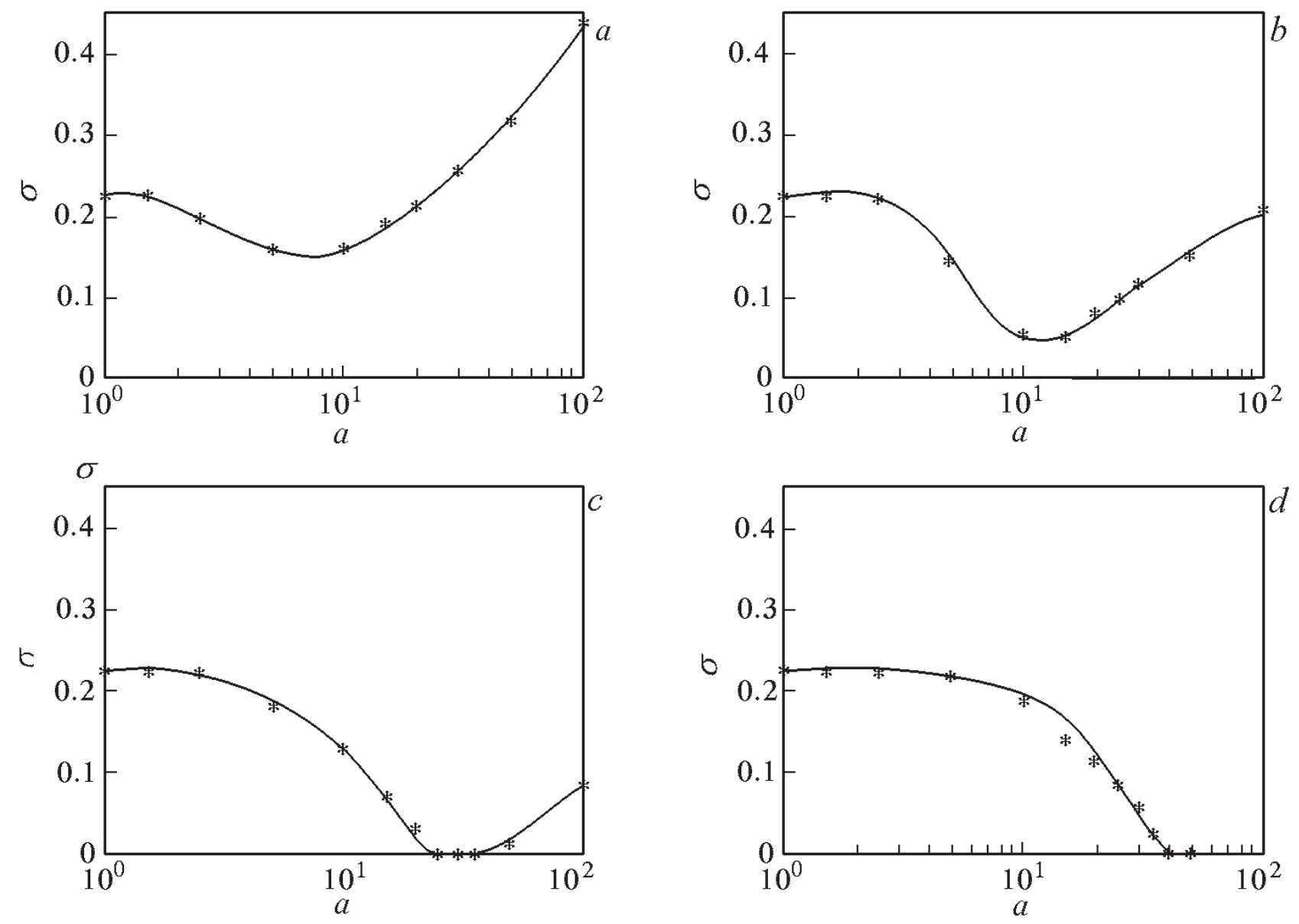

Fig. 20. 


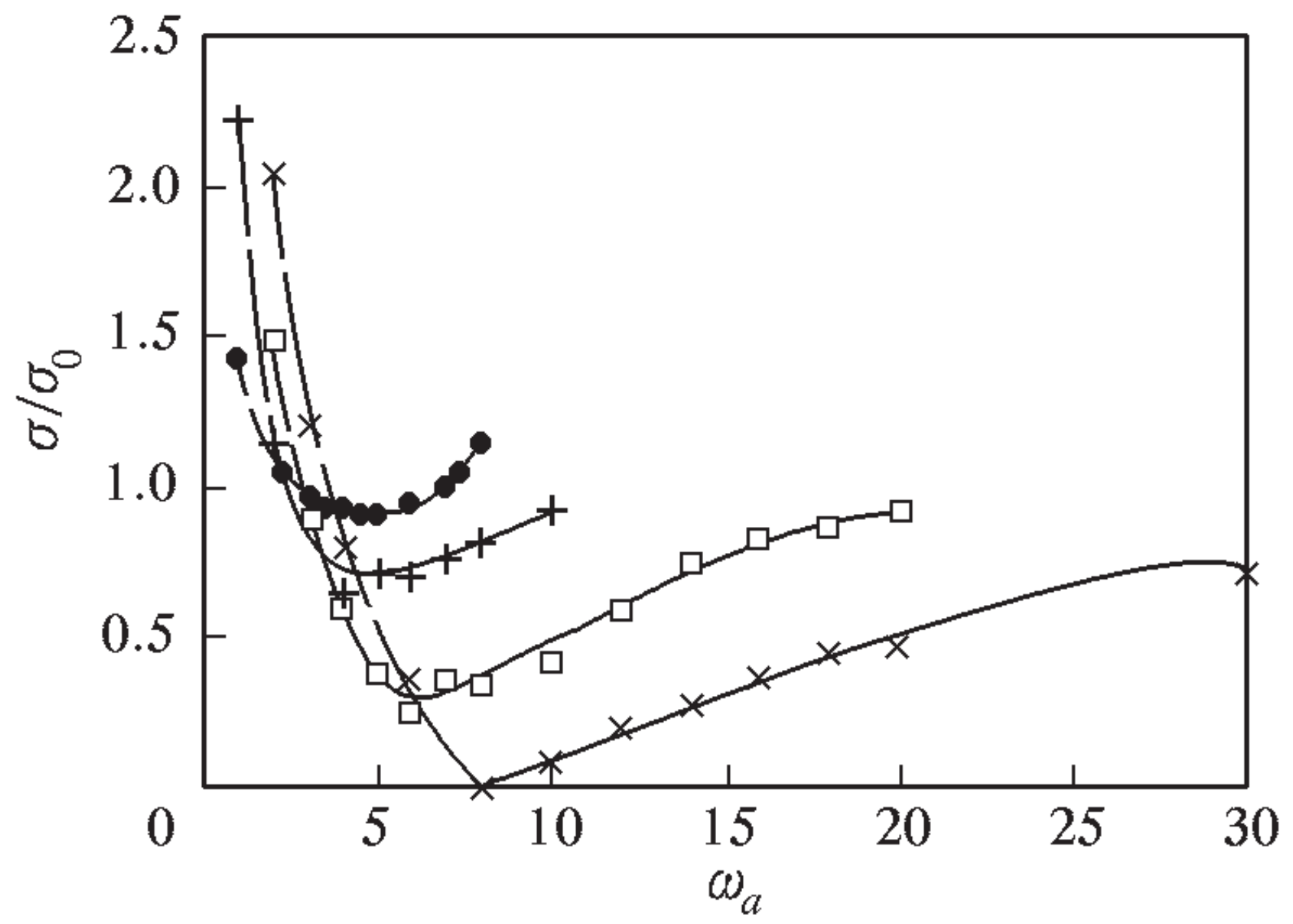

Fig. 21.

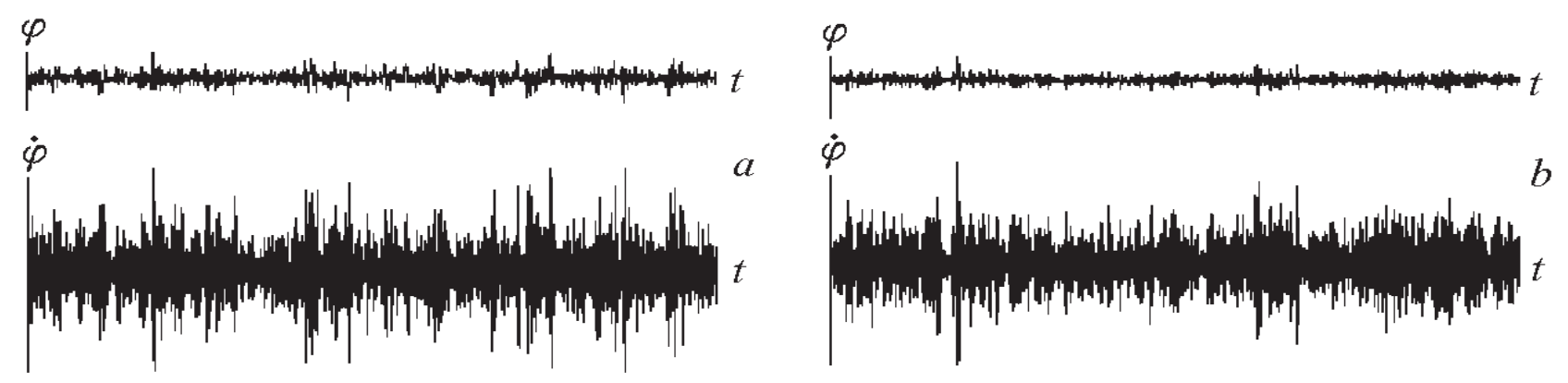

Fig. 22. 

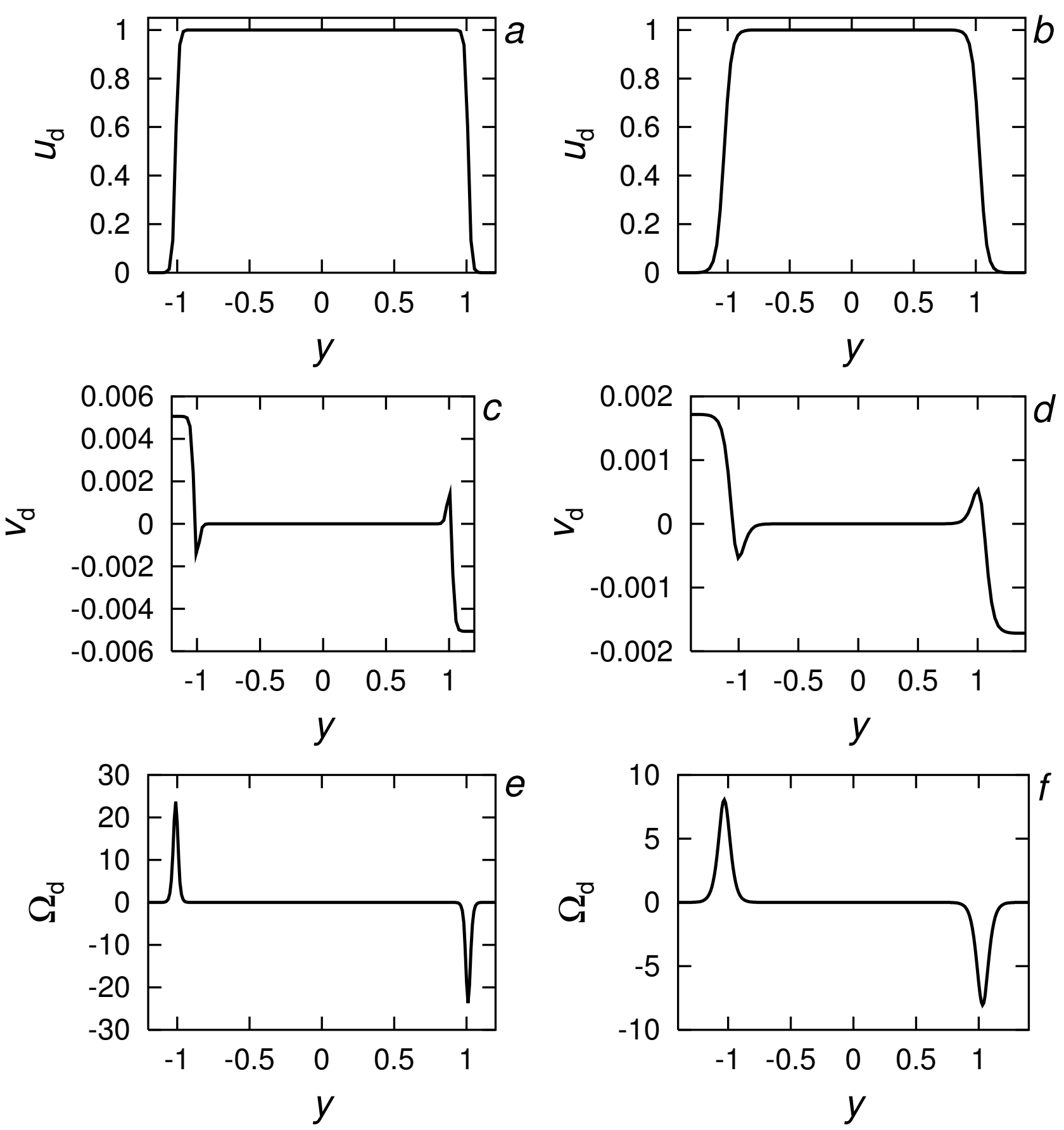

Fig. 23. 

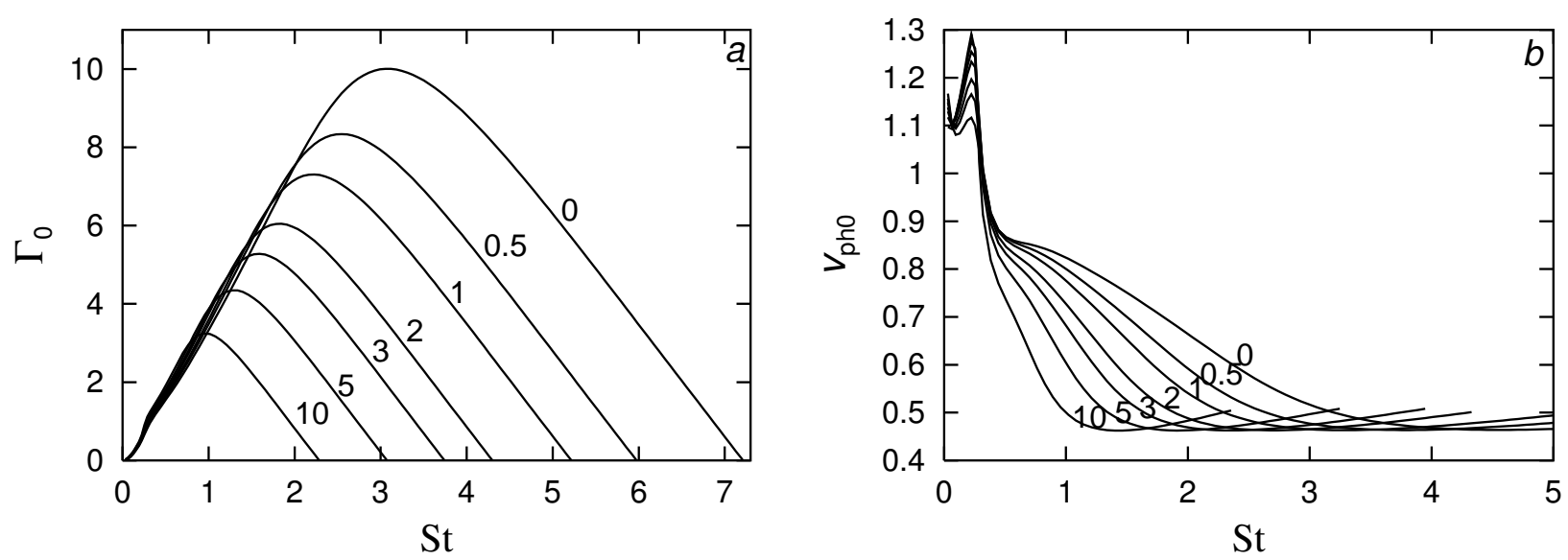

Fig. 24.
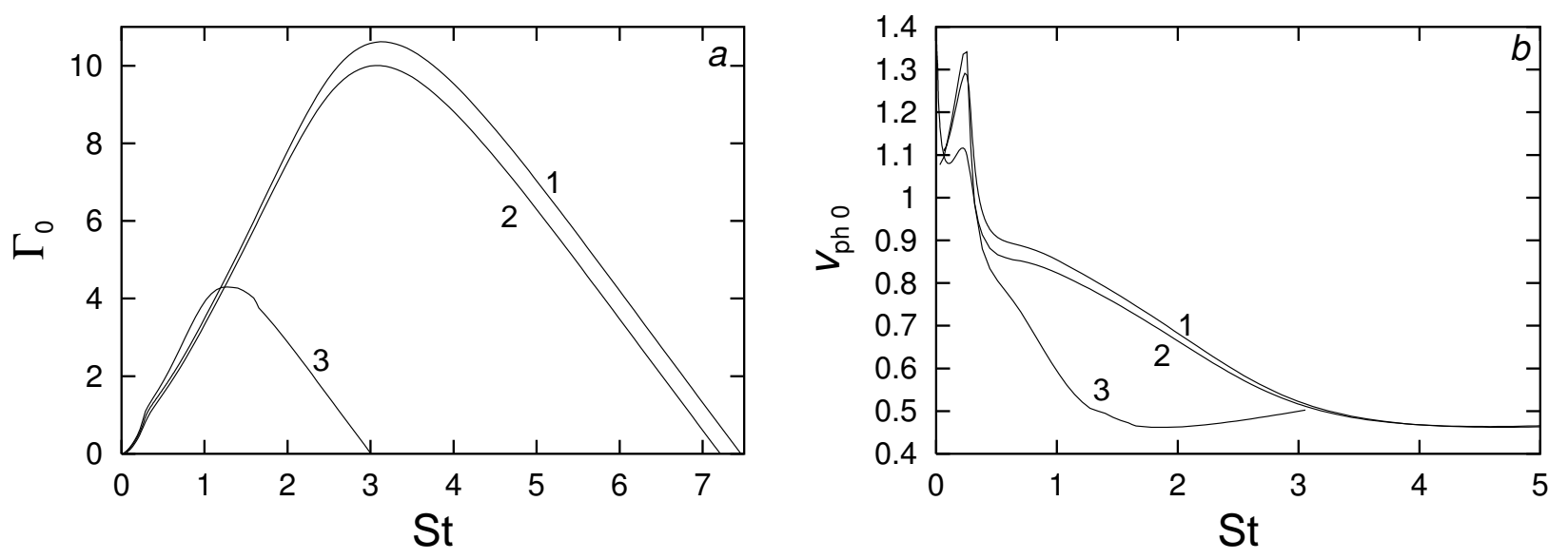

Fig. 25. 

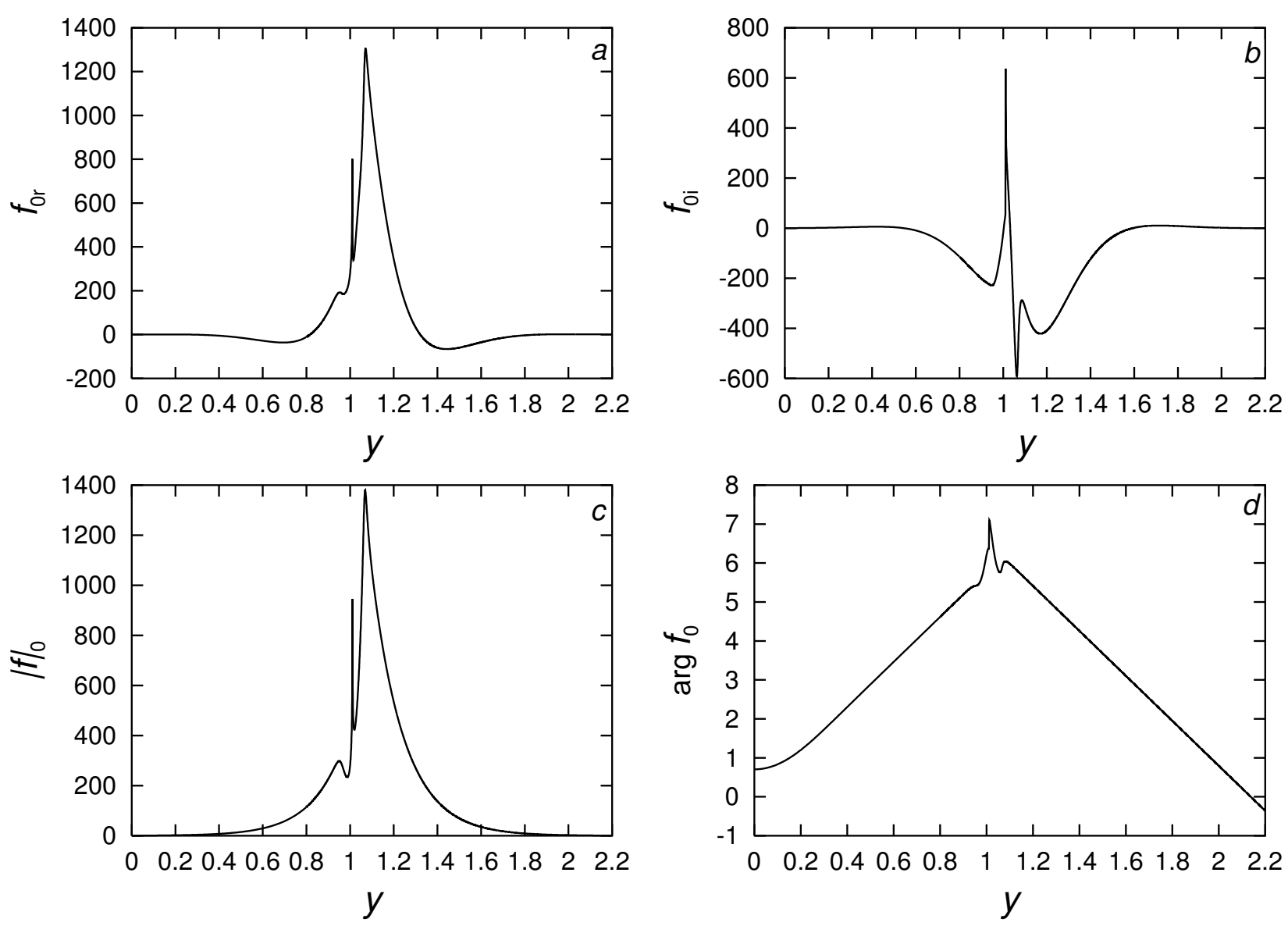

Fig. 26. 

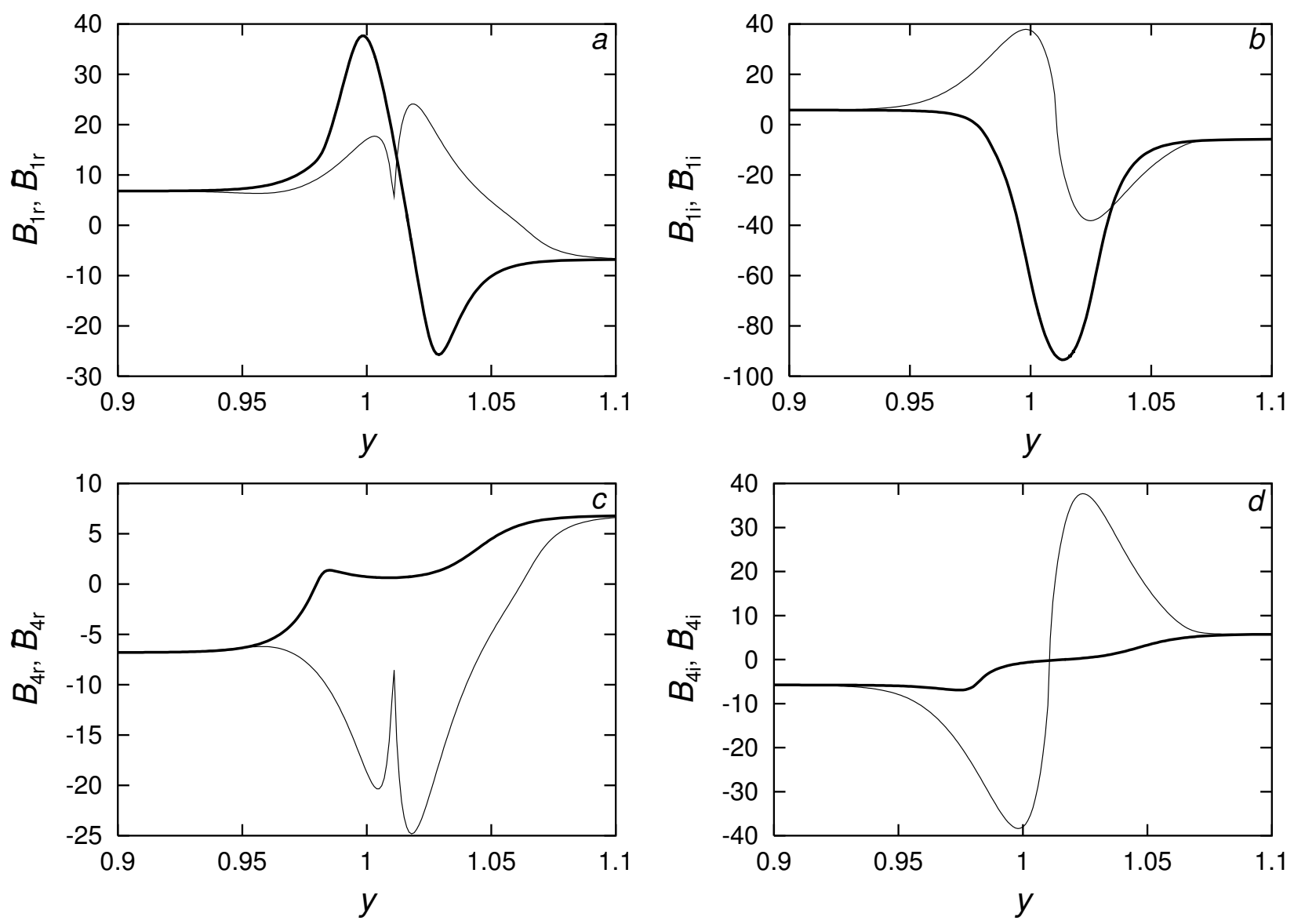

Fig. 27. 

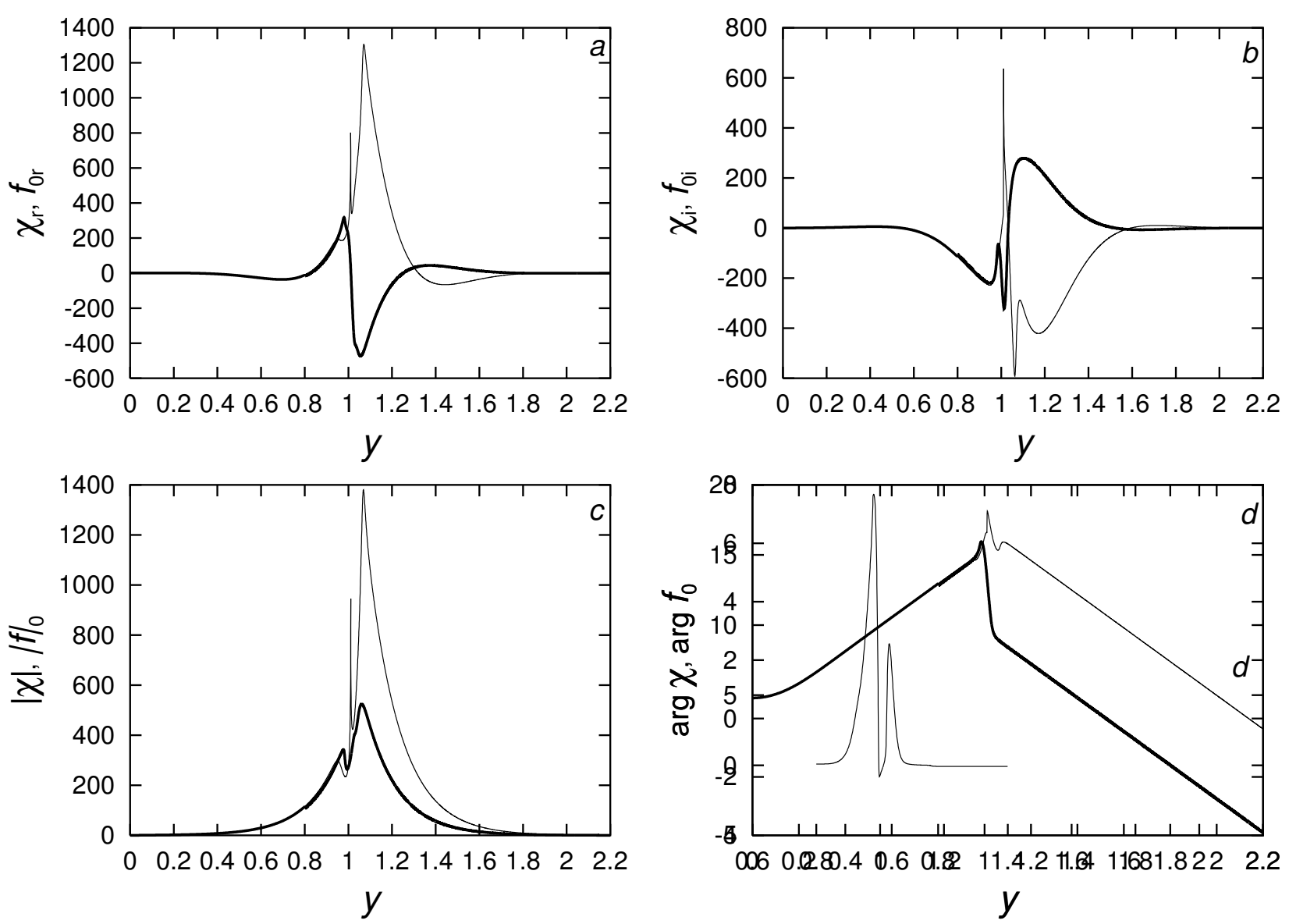

Fig. 28. 

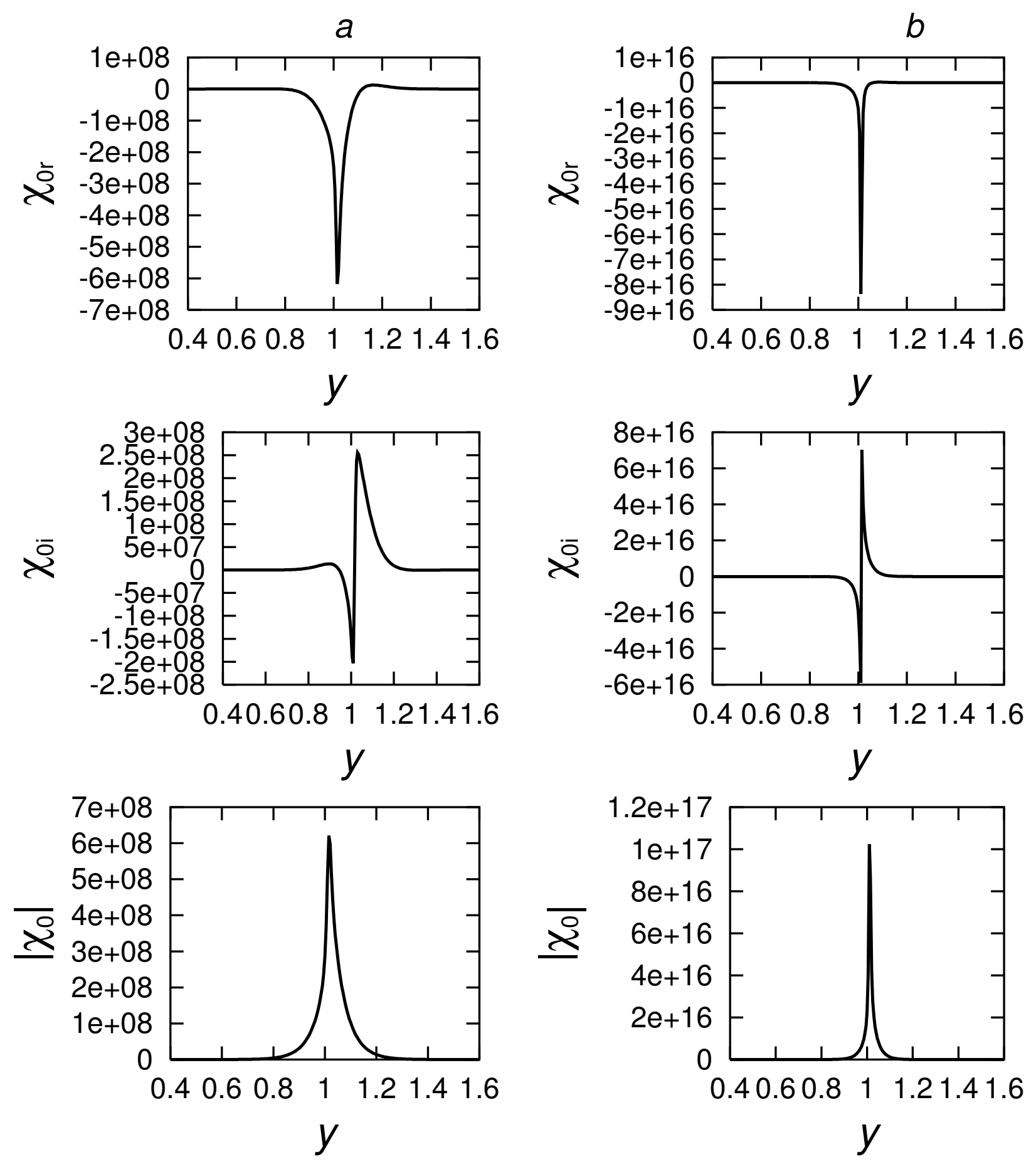

Fig. 29. 

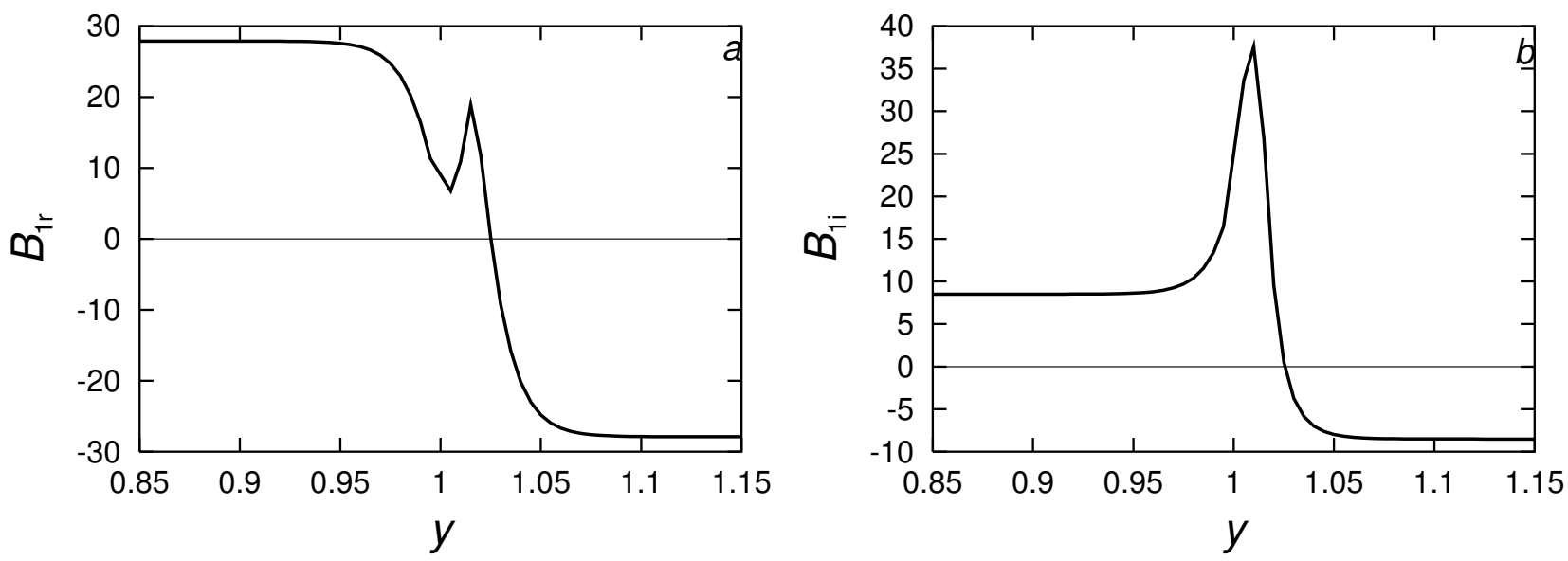

Fig. 30.
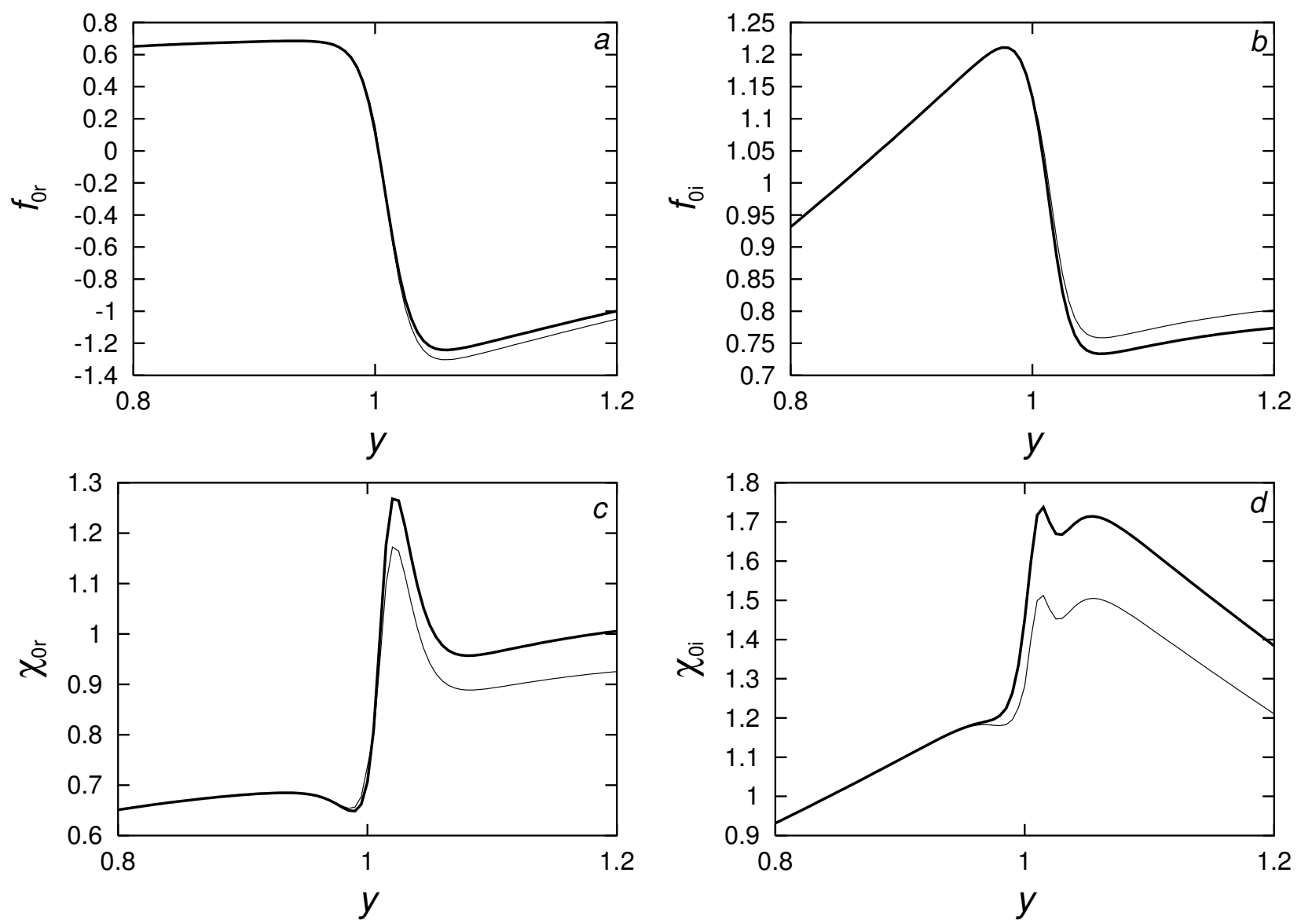

Fig. 31. 

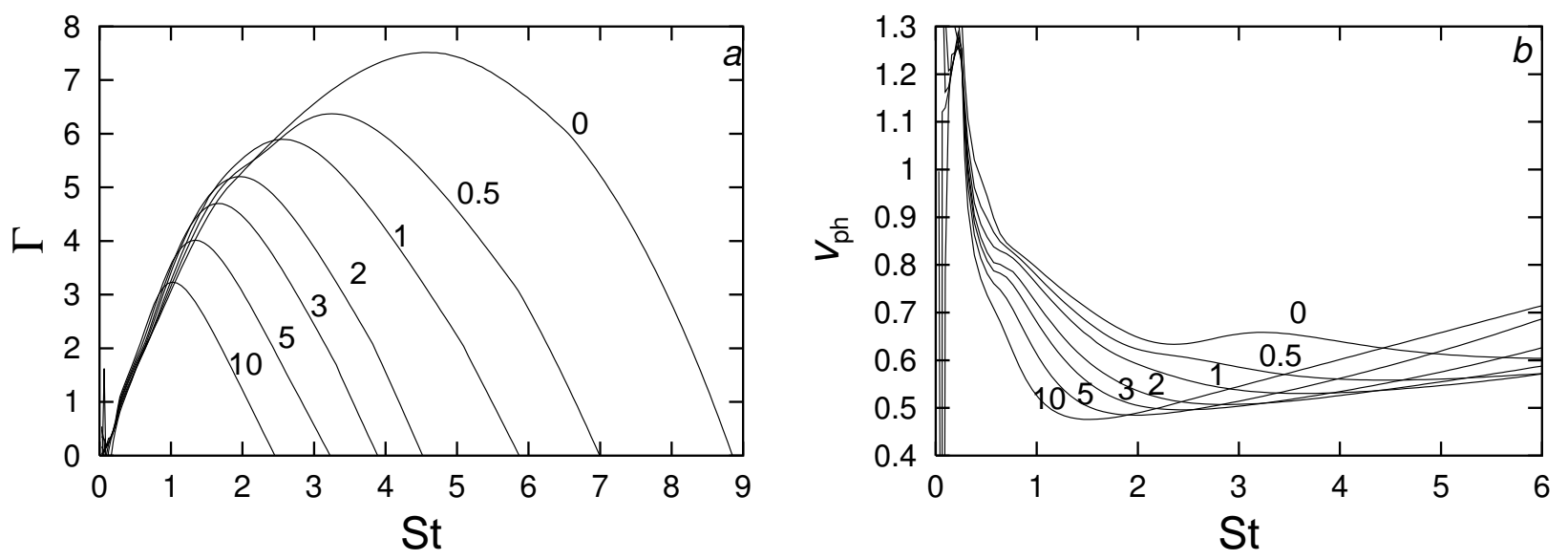

Fig. 32 .
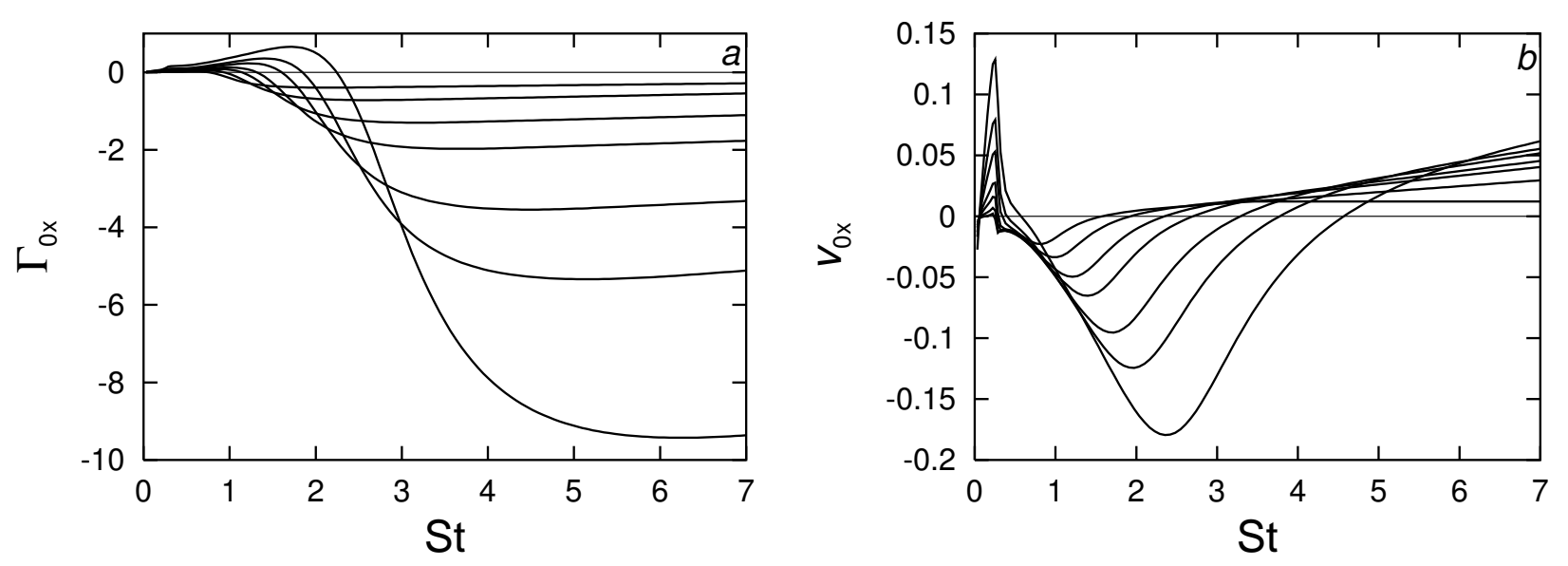

Fig. 33. 


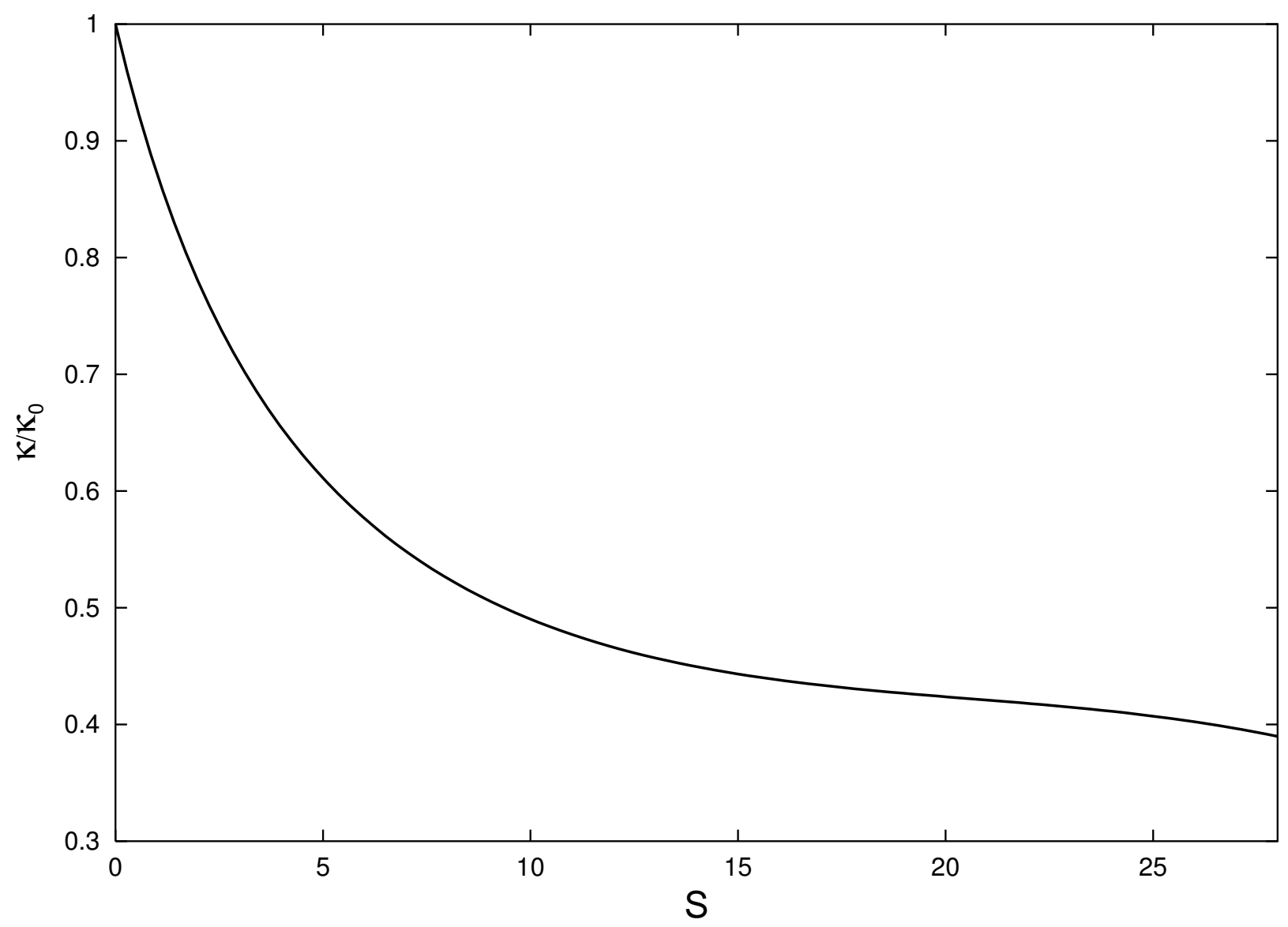

Fig. 34 .
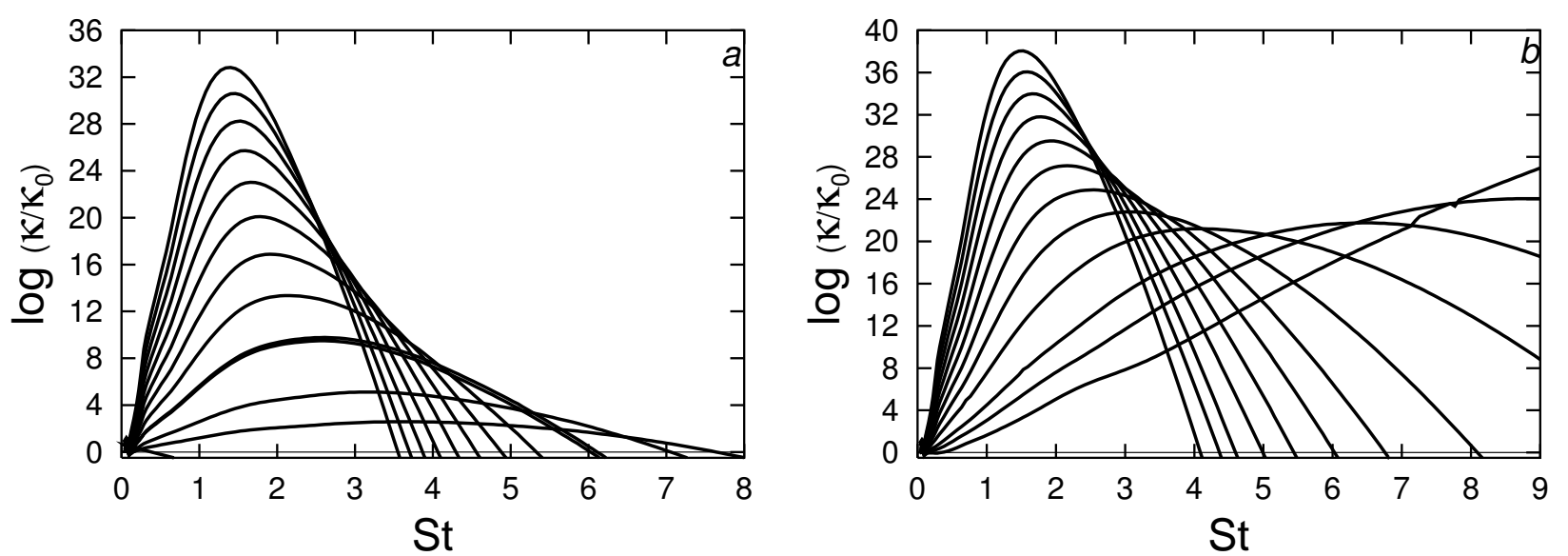

Fig. 35 . 

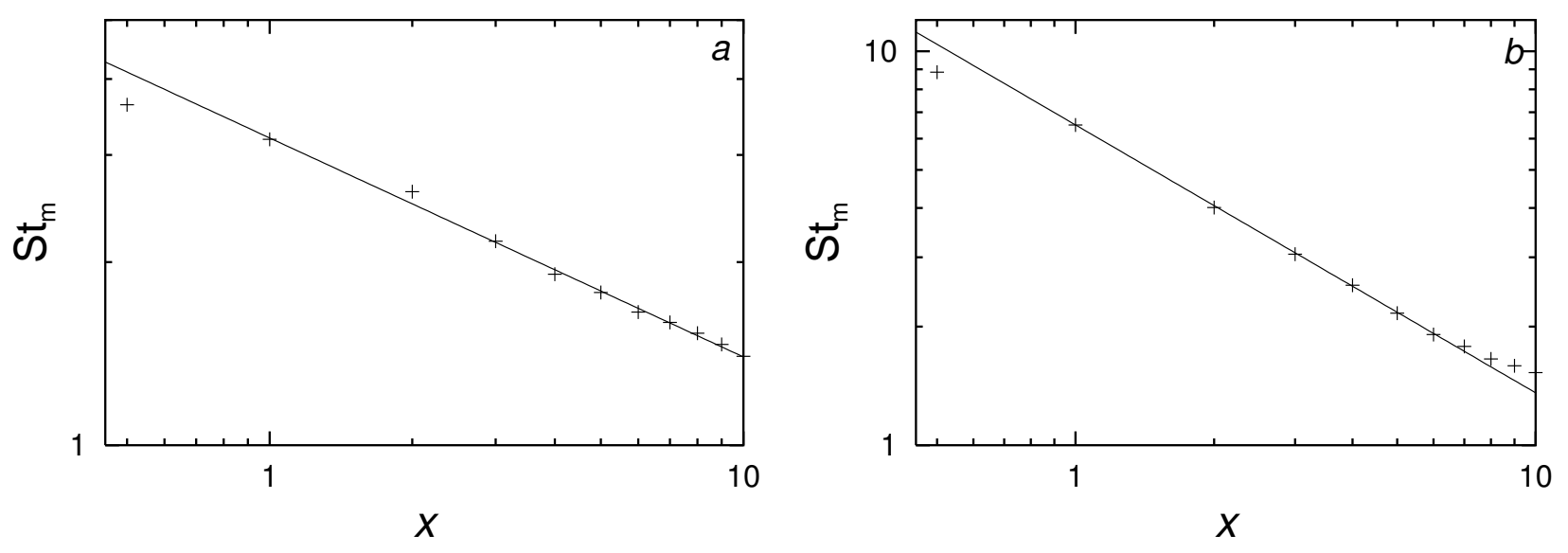

Fig. 36 .
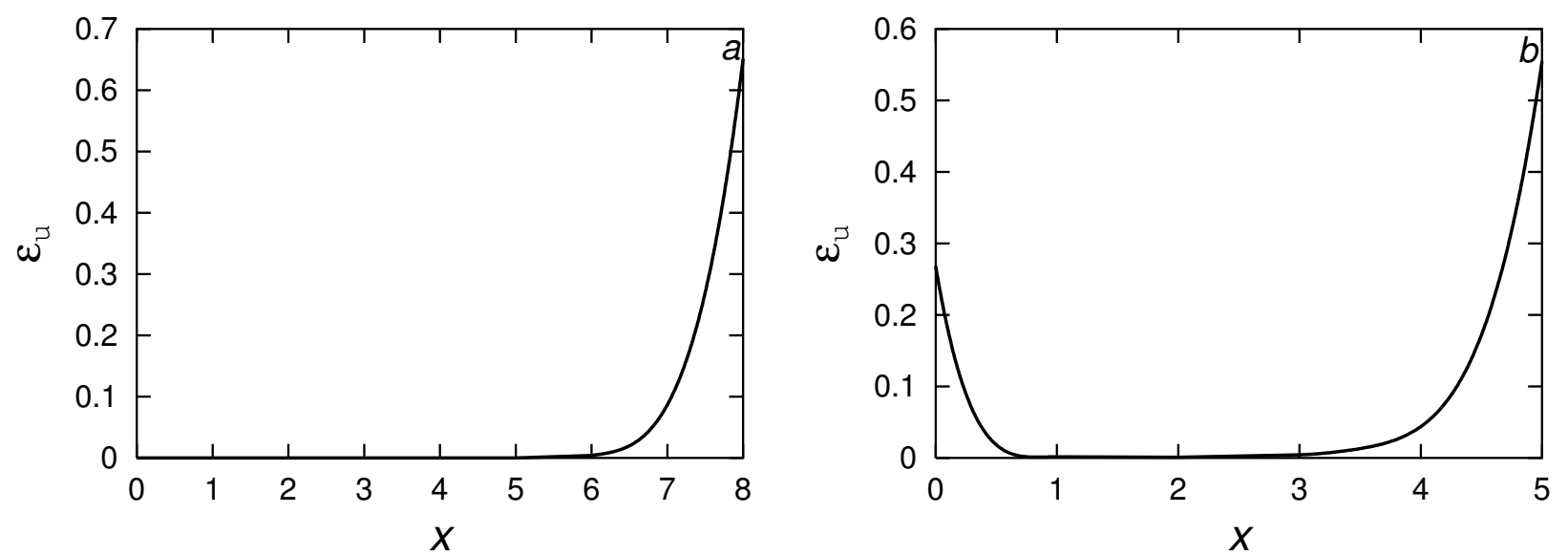

Fig. 37. 

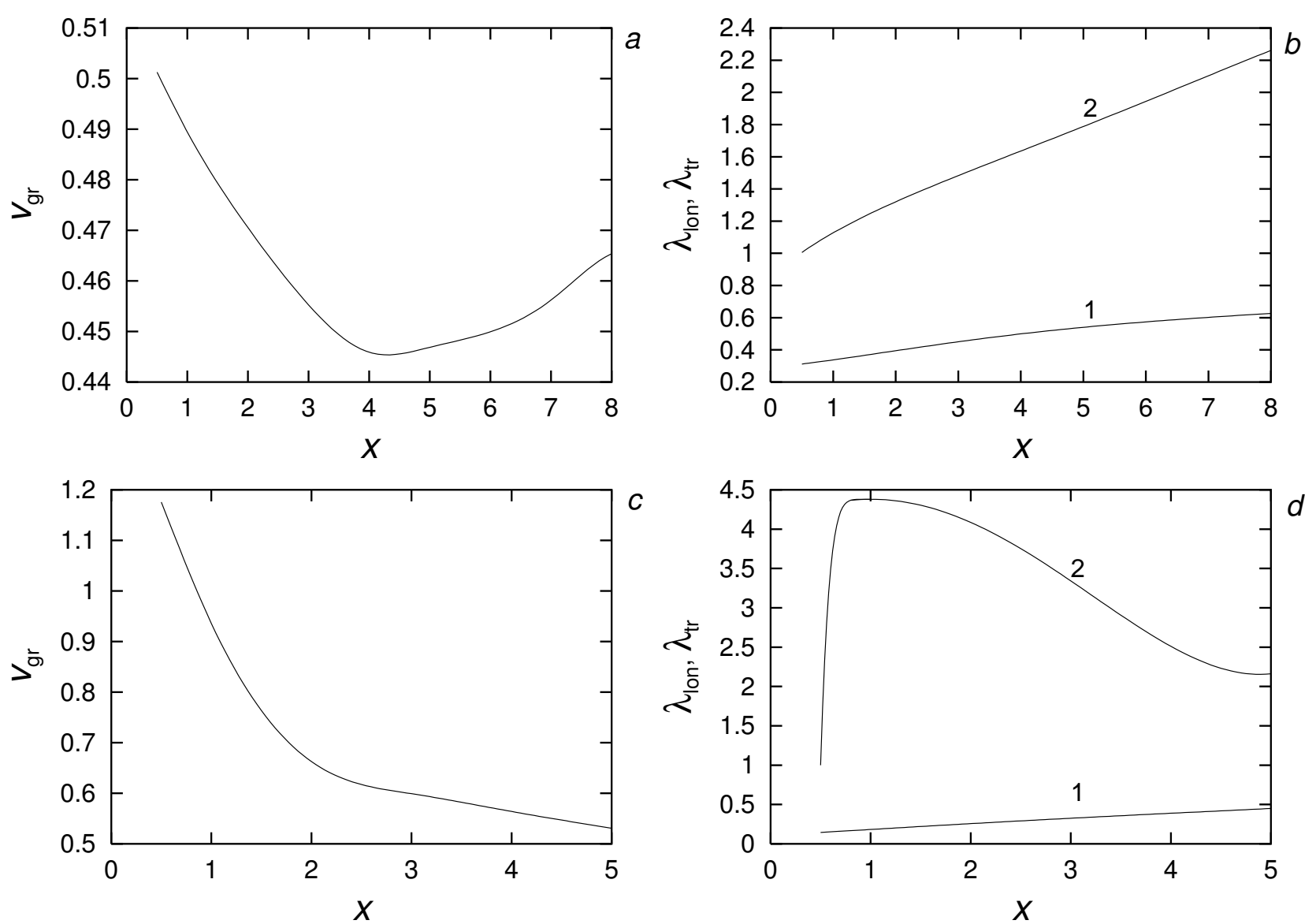

Fig. 38.
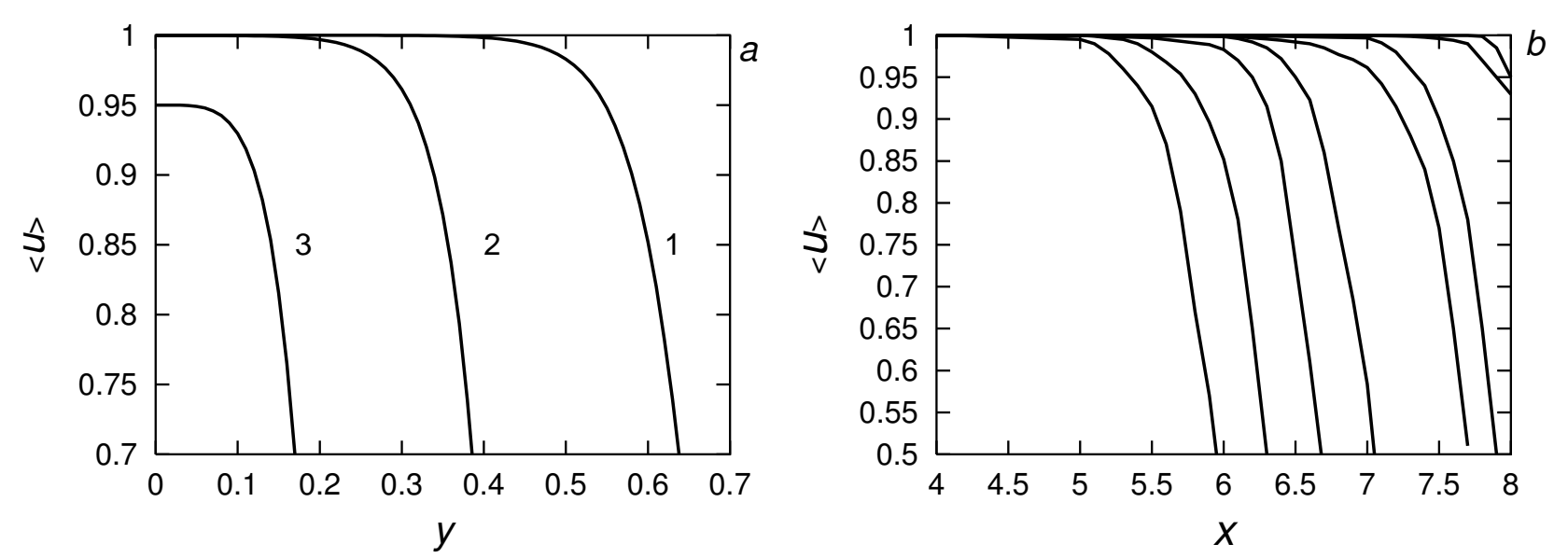

Fig. 39. 

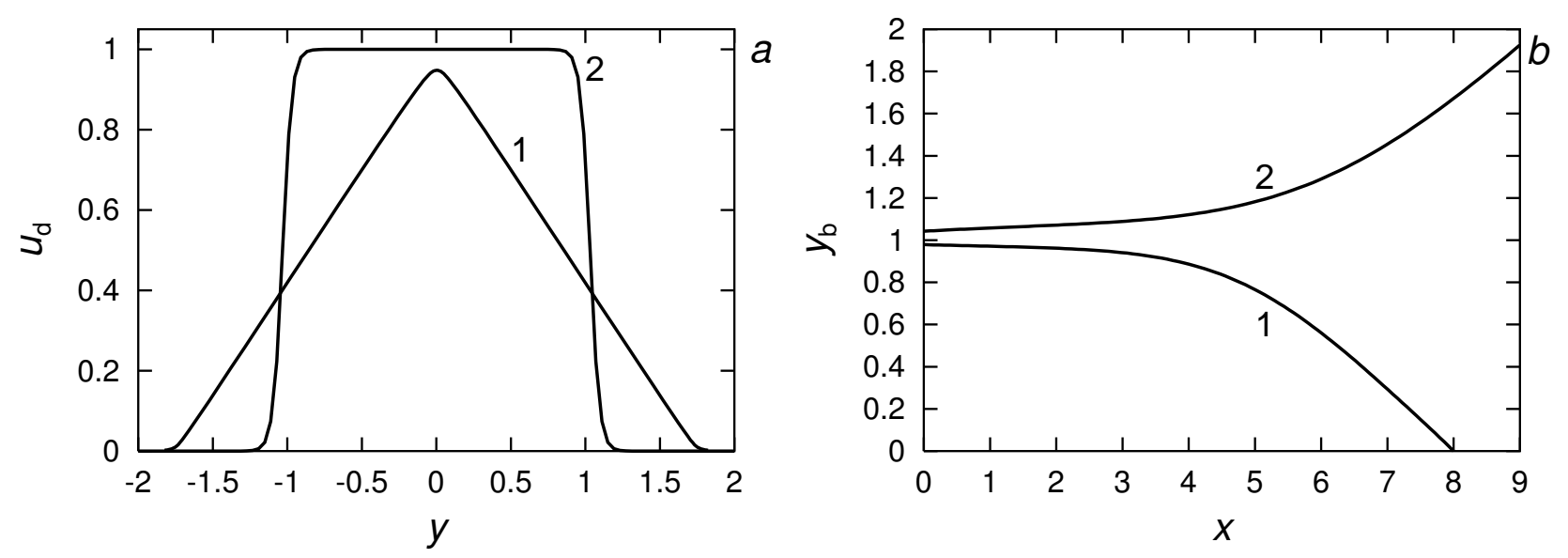

Fig. 40.
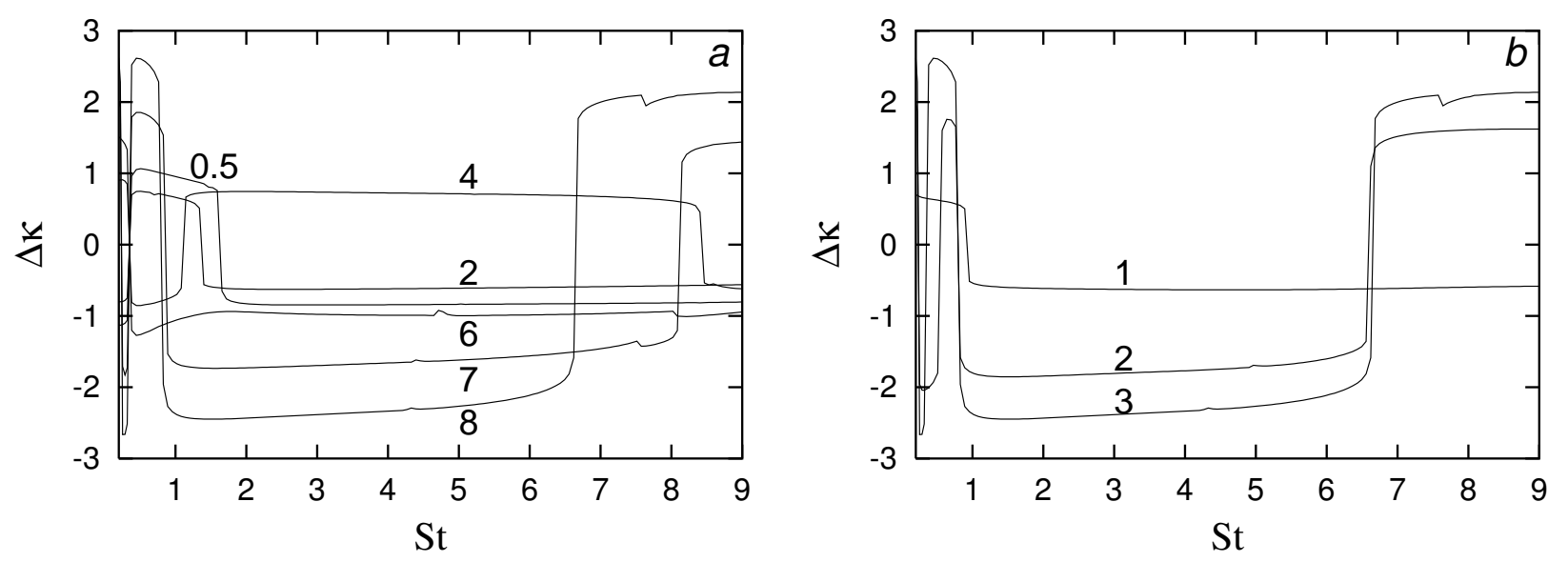

Fig. 41. 


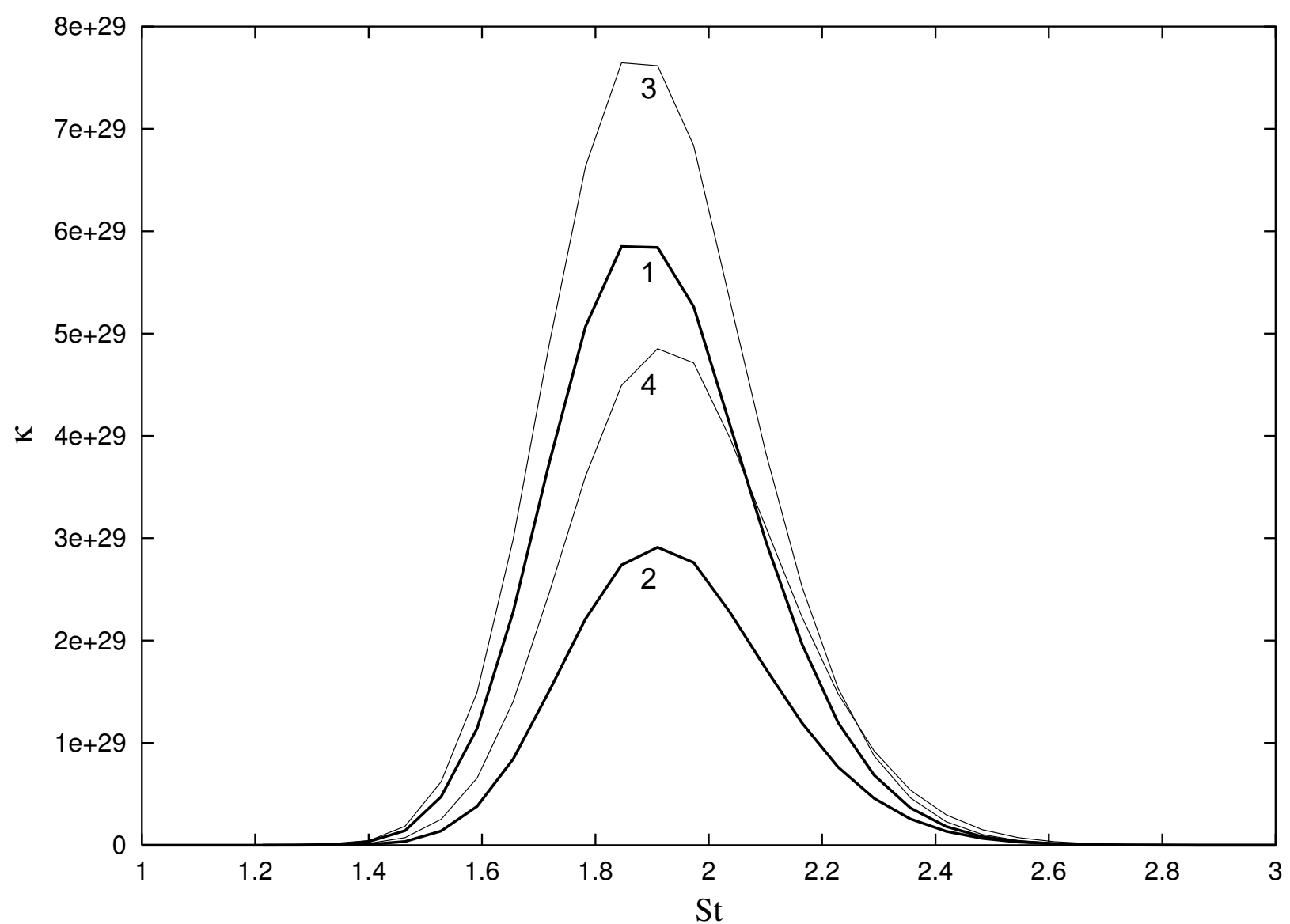

Fig. 42. 